\title{
Adaptiveness of the empirical distribution of residuals in semi- parametric conditional location scale models
}

Christian Francq and Jean-Michel Zakoïan

CREST and University of Lille, 5 Avenue Henri Le Chatelier, 91120 Palaiseau, France.

E-mail: christian.francq@univ-lille3.fr, zakoian@ensae.fr

Summary. This paper addresses the problem of deriving the asymptotic distribution of the empirical distribution function $\widehat{F}_{n}$ of the residuals in a general class of time series models, including conditional mean and conditional heteroscedaticity, whose independent and identically distributed errors have unknown distribution $F$. We show that, for a large class of time series models (including the standard ARMA-GARCH), the asymptotic distribution of $\sqrt{n}\left\{\widehat{F}_{n}(\cdot)-F(\cdot)\right\}$ is impacted by the estimation but does not depend on the model parameters. It is thus neither asymptotically estimation free, as is the case for purely linear models, nor asymptotically model dependent, as is the case for some nonlinear models. The asymptotic stochastic equicontinuity is also established. We consider an application to the estimation of the conditional Value-at-Risk. 


\section{Introduction}

For independent variables $\eta_{1}, \ldots, \eta_{n}$ with common distribution $F$, the celebrated Glivenko-Cantelli theorem states the uniform almost sure convergence of the empirical distribution,

$$
\sup _{x \in \mathbb{R}}\left|F_{n}(x)-F(x)\right| \rightarrow 0, \quad \text { a.s. }
$$

where $F_{n}(x)=\frac{1}{n} \sum_{t=1}^{n} 1_{\left\{\eta_{t} \leq x\right\}}$ for all $x$. The usual Central Limit Theorem (CLT) shows that $\sqrt{n}\left\{F_{n}(x)-F(x)\right\}$ is asymptotically $\mathcal{N}(0, F(x)(1-F(x))$ distributed without any further assumption. Moreover, under the assumption that $F$ is continuous, the sequence $\left\{\sqrt{n}\left[F_{n}(\cdot)-F(\cdot)\right]\right\}$ is stochastically equicontinuous, in the sense that $\sqrt{n}\left\{F_{n}\left(x_{n}\right)-F\left(x_{n}\right)\right\} \stackrel{\mathcal{L}}{\rightarrow} \mathcal{N}(0, F(x)(1-F(x))$ for any sequence $\left(x_{n}\right)$ converging to $x$ in probability, for any $x$ (see Billingsley (1968, Section 22) and Andrews (1994)). The latter convergence in distribution has important statistical applications, for instance the derivation of the asymptotic distribution of the empirical quantiles (see below).

Time series observations are rarely independent and identically distributed (iid), but time series models often involve iid innovations. Modern time series analysis often relies on semi-parametric models in which the distribution $F$ of the innovations $\eta_{t}$ is not fully specified. Many such models are of the location-scale form $y_{t}=m_{t}\left(\boldsymbol{\theta}_{0}\right)+\sigma_{t}\left(\boldsymbol{\theta}_{0}\right) \eta_{t}$, where $m_{t}$ and $\sigma_{t}$ are functions of the past observations $y_{t-i}, i>0$, and the sequence $\left(\eta_{t}\right)$ is iid. Consistent estimation of the parameter $\boldsymbol{\theta}_{0}$ can be achieved by several methods. In particular, the Quasi-Maximum Likelihood (QML) estimation method generally relies on a Gaussian criterion - written as if the distribution $F$ was Gaussian but provides consistent estimators while remaining agnostic concerning the true distribution.

Even if no distributional assumption is made during the estimation phase, using semi-parametric time series models may require information about the errors distribution $F$. For instance, in the location-scale model the conditional Value-at-Risk (VaR) at level $\alpha \in(0,1)$ is equal to $m_{t}+\sigma_{t} \xi_{\alpha}$ where $\xi_{\alpha}$ is the $\alpha$-quantile of $F$. Information about $F$ can be obtained via the empirical distribution function of the residuals (e.d.f.r.) $\widehat{F}_{n}$. Consistent estimation of the parameters produce residuals which are obviously not iid but converge to the innovations. One question of importance is whether the previous properties on $F_{n}$ apply to $\widehat{F}_{n}$, i.e. when innovations are replaced by residuals.

For many applications, the asymptotic distribution of the sequence $\left\{\sqrt{n}\left[\widehat{F}_{n}(\cdot)-F(\cdot)\right]\right\}$ is required. In the statistical literature, two kinds of results have been established. In some situations, the asymptotic distribution is asymptotically estimation free (AEF), in the sense that, asymptotically, $\sqrt{n}\left\{\widehat{F}_{n}(\cdot)-F(\cdot)\right\} \stackrel{d}{=} \sqrt{n}\left\{F_{n}(\cdot)-F(\cdot)\right\}$. Such results go back to Boldin (1982), for the $\operatorname{AR}(p)$ process, and have been generalized to linear processes by Kreiss (1991) and Bai (1994). On the other hand, existing results for nonlinear processes show that the distribution of $\sqrt{n}\left\{\widehat{F}_{n}(\cdot)-F(\cdot)\right\}$ can be much more complicated. It can be asymptotically model dependent (AMD), in the sense 
that the asymptotic variance depends on both the law of the innovations $F$ and the parameter $\boldsymbol{\theta}_{0}$. For different classes of conditionally heteroscedatic time series, the asymptotic distribution was investigated by Boldin (1998), and Lee and Taniguchi (2005) among others, and for the squared residuals by Horváth, Kokoszka and Teyssière (2001) and Berkes and Horváth (2003). In all these works, the distribution of the statistics involving the residuals is AMD. To overcome the AMD, Koul and Ling (2006) proposed a modification of the Kolmogorov-Smirnov test based on residuals. See Berkes and Horváth (2002) for a review of the asymptotic behaviour of e.d.f.r.

The main contribution of this paper is to show that, for a large class of time series models (including the standard ARMA-GARCH), the asymptotic distribution of $\sqrt{n}\left\{\widehat{F}_{n}(\cdot)-F(\cdot)\right\}$ is neither AEF nor AMD. In some cases, which we are able to characterize, the distribution is asymptotically model free (AMF), however it is impacted by the estimation, i.e. it is asymptotically estimation dependent (AED), though only through the errors distribution. The AMF property simplifies dramatically the use of such asymptotic results in practical applications based on potentially complex time series models. On the other hand, the AED property tells us that usual statistics available for observed iid processes cannot be directly employed.

We start by considering volatility modelling and QML estimation, which constitutes the most widely used framework for financial returns. The absence of a conditional mean and the choice of a specific estimation method allows us to derive finer results. However, from an extended perspective, we will also consider conditional location-scale models in which both first conditional moments are involved, and more general estimation methods. We will also investigate an application to the VaR estimation.

The rest of the paper is organized as follows. In the next section we introduce a general parametric volatility model and the main assumptions ensuring the consistency of the e.d.f.r. Next, we state our first main result, establishing the asymptotic distribution of the e.d.f.r. Section 3 considers the extension to conditional location-scale models. The application to VaR is developed in Section 4. In particular, we provide conditions for the strong consistency and asymptotic normality of the residuals quantiles. Section 5 concludes. Most proofs are postponed to Section 6 or to an Appendix.

\section{Empirical distribution of the residuals for volatility models}

In this section, we focus on conditional scale models of the multiplicative form

$$
\epsilon_{t}=\sigma_{t} \eta_{t}, \quad \sigma_{t}=\sigma\left(\epsilon_{t-1}, \epsilon_{t-2}, \ldots ; \boldsymbol{\theta}_{0}\right),
$$

where $\left(\eta_{t}\right)$ is a sequence of iid random variables, $E\left(\eta_{t}^{2}\right)=1, \boldsymbol{\theta}_{0}$ is a vector of unknown coefficients which belongs to a compact parameter set $\Theta \subset \mathbb{R}^{d}$, and $\sigma$ is a positive function. Note that we do 
not assume that $E\left(\eta_{t}\right)=0$.

For instance, the standard $\operatorname{GARCH}(p, q)$ model writes

$$
\epsilon_{t}=\sigma_{t} \eta_{t}, \quad \sigma_{t}^{2}=\omega_{0}+\sum_{i=1}^{q} \alpha_{0 i} \epsilon_{t-i}^{2}+\sum_{j=1}^{p} \beta_{0 j} \sigma_{t-j}^{2}
$$

where $\boldsymbol{\theta}_{0}=\left(\omega_{0}, \alpha_{01}, \ldots, \beta_{0 p}\right)^{\prime}$ satisfies $\omega_{0}>0, \alpha_{0 i} \geq 0, \beta_{0 j} \geq 0$. Under strict stationarity, invertibility of the polynomial $\mathcal{B}(z)=1-\sum_{j=1}^{p} \beta_{0 j} z^{j}$ holds and the volatility $\sigma_{t}^{2}$ can indeed be written as a linear function of the past values of $\epsilon_{t}^{2}$.

We start by considering the Gaussian QML estimation method for which explicit conditions for Consistency and Asymptotic Normality (CAN) can be displayed. More general estimators will be considered in Section 3. The setup can be described as follows. Given observations $\epsilon_{1}, \ldots, \epsilon_{n}$, and using arbitrary initial values $\tilde{\epsilon}_{i}$ for $i \leq 0$, we define for any $\boldsymbol{\theta} \in \mathbf{\Theta}$,

$$
\tilde{\sigma}_{t}(\boldsymbol{\theta})=\sigma\left(\epsilon_{t-1}, \epsilon_{t-2}, \ldots, \epsilon_{1}, \tilde{\epsilon}_{0}, \tilde{\epsilon}_{-1}, \ldots ; \boldsymbol{\theta}\right)
$$

which will be used as a proxy of $\sigma_{t}(\boldsymbol{\theta})=\sigma\left(\epsilon_{t-1}, \epsilon_{t-2}, \ldots, \epsilon_{1}, \epsilon_{0}, \epsilon_{-1}, \ldots ; \boldsymbol{\theta}\right)$.

A Gaussian QML estimator (QMLE) of $\boldsymbol{\theta}_{0}$ is defined as

$$
\widehat{\boldsymbol{\theta}}_{n}=\arg \min _{\boldsymbol{\theta} \in \boldsymbol{\Theta}} \frac{1}{n} \sum_{t=1}^{n} \tilde{\ell}_{t}(\boldsymbol{\theta}), \quad \tilde{\ell}_{t}(\boldsymbol{\theta})=\frac{\epsilon_{t}^{2}}{\tilde{\sigma}_{t}^{2}(\boldsymbol{\theta})}+\log \tilde{\sigma}_{t}^{2}(\boldsymbol{\theta}) .
$$

Let $K>0$ be a generic constant or random variable measurable with respect to $\mathcal{F}_{0}$, where $\mathcal{F}_{t}$ denotes the $\sigma$-algebra generated by $\left\{\eta_{s}, s \leq t\right\}$. Let $\rho \in(0,1)$. We shall assume the following.

A1: $\left(\epsilon_{t}\right)$ is a strictly stationary, non-anticipative (i.e. $\epsilon_{t} \in \mathcal{F}_{t}$ ) and ergodic solution of Model (1). Moreover, $E\left|\sigma_{t}\right|^{r}<\infty$ for some $r>0$.

A2: For any real sequence $\left(x_{i}\right)$, the function $\boldsymbol{\theta} \mapsto \sigma\left(x_{1}, x_{2}, \ldots ; \boldsymbol{\theta}\right)$ is continuous differentiable. Almost surely, $\sigma_{t}(\boldsymbol{\theta}) \in(\underline{\omega}, \infty]$ for any $\boldsymbol{\theta} \in \boldsymbol{\Theta}$ and for some $\underline{\omega}>0$. Moreover, $\sigma_{t}\left(\boldsymbol{\theta}_{0}\right) / \sigma_{t}(\boldsymbol{\theta})=$ 1 a.s. iff $\boldsymbol{\theta}=\boldsymbol{\theta}_{0}$.

A3: $\sup _{\boldsymbol{\theta} \in \boldsymbol{\Theta}}\left|\sigma_{t}(\boldsymbol{\theta})-\tilde{\sigma}_{t}(\boldsymbol{\theta})\right| \leq K \rho^{t}$.

A4: There exists a neighborhood $V\left(\boldsymbol{\theta}_{0}\right)$ of $\boldsymbol{\theta}_{0}$ such that $E\left(\sup _{\boldsymbol{\theta} \in V\left(\boldsymbol{\theta}_{0}\right)} \frac{\sigma_{t}\left(\boldsymbol{\theta}_{0}\right)}{\sigma_{t}(\boldsymbol{\theta})}\right)^{r}<\infty$ and $E \sup _{\boldsymbol{\theta} \in V\left(\boldsymbol{\theta}_{0}\right)}\left\|\boldsymbol{D}_{t}(\boldsymbol{\theta})\right\|^{r}<\infty$, where $\boldsymbol{D}_{t}(\boldsymbol{\theta})=\sigma_{t}^{-1}(\boldsymbol{\theta}) \partial \sigma_{t}(\boldsymbol{\theta}) / \partial \boldsymbol{\theta}$.

Assumptions A1-A3 are a set of conditions ensuring the strong consistency of $\widehat{\boldsymbol{\theta}}_{n}$ (see Francq and Zakoian (2004, 2015)). Assumption A4 is introduced to control the difference between the innovations and the residuals. ${ }^{1}$

${ }^{1}$ For the classical $\operatorname{GARCH}(p, q)$ model, under invertibility conditions on the lag polynomial $\mathcal{B}_{\boldsymbol{\theta}}(z)=1-$ $\sum_{j=1}^{p} \beta_{j} z^{j}$ and assuming that the law of $\eta_{t}^{2}$ is nondegenerate, $\mathbf{A 1}-\mathbf{A} 4$ reduce to the first part of $\mathbf{A} \mathbf{1}$. 
Let the residuals $\widehat{\eta}_{t}=\epsilon_{t} / \tilde{\sigma}_{t}\left(\widehat{\boldsymbol{\theta}}_{n}\right)$. We note that, at least for $t$ large enough, $\tilde{\sigma}_{t}\left(\widehat{\boldsymbol{\theta}}_{n}\right) \geq \underline{\omega}$ by A2A3. We start by establishing a Glivenko-Cantelli result for the e.d.f.r. $\widehat{F}_{n}(x)=\frac{1}{n} \sum_{t=1}^{n} 1_{\left\{\widehat{\eta}_{t} \leq x\right\}} \cdot{ }^{2}$ The following assumption is simply denoted $\mathbf{A 5}$ when it holds for all $x \in \mathbb{R}$.

A5(x): For $x \in \mathbb{R}$, the cdf $F$ of $\eta$ is Lipschitz continuous in a neighborhood of $x$.

\section{Theorem 1. Under A1-A4,}

(a) If $\mathbf{A 5}(x)$ holds for $x \in \mathbb{R}$, we have $\left|\widehat{F}_{n}(x)-F(x)\right| \rightarrow 0$ a.s.

(b) If $\mathbf{A} 5$ holds we have $\sup _{x \in \mathbb{R}}\left|\widehat{F}_{n}(x)-F(x)\right| \rightarrow 0$ a.s.

Notice that the previous uniform convergence was established by Stute (2001) in the case of $\operatorname{ARCH}(q)$ processes. It is known (see for instance Stute and Schumann (1980)) that in the case of a stationary ergodic sequence, the Glivenko-Cantelli theorem is valid without any assumption on $F$. The following example shows that $\mathbf{A 5}$ is required for the consistency of the e.d.f.r.

Example 1 (Glivenko-Cantelli in failure). Consider the scale model $\epsilon_{t}=\sigma \eta_{t}, \sigma>0$, where $\eta_{t}$ is distributed over $\{-\sqrt{2}, 0, \sqrt{2}\}$ with $P\left(\eta_{t}=-\sqrt{2}\right)=P\left(\eta_{t}=\sqrt{2}\right)=1 / 4$. Let $\widehat{\sigma}_{n}$ be a consistent estimator of $\sigma$. The residuals $\widehat{\eta}_{t}=\frac{\sigma}{\widehat{\sigma}_{n}} \eta_{t}$ have the e.d.f.r.

$$
\widehat{F}_{n}(x)=\left(\frac{1}{n} \sum_{t=1}^{n} \mathbb{1}_{\epsilon_{t}<0}\right) \mathbb{1}_{-\sqrt{2} \frac{\sigma}{\widetilde{\sigma}_{n}} \leq x<0}+\left(\frac{1}{n} \sum_{t=1}^{n} \mathbb{1}_{\epsilon_{t} \leq 0}\right) \mathbb{1}_{0 \leq x<\sqrt{2} \frac{\sigma}{\tilde{\sigma}_{n}}}+\mathbb{1}_{x \geq \sqrt{2}} \frac{\sigma}{\widehat{\sigma}_{n}} .
$$

It follows that $\sup _{x \in \mathbb{R}}\left|\widehat{F}_{n}(x)-F(x)\right| \geq\left|\widehat{F}_{n}(-\sqrt{2})-F(-\sqrt{2})\right|=1 / 4$ whenever $\sigma<\widehat{\sigma}_{n}$, which has a non vanishing probability for the $Q M L$. Indeed, we have $\widehat{\sigma}_{n}^{2}=\frac{1}{n} \sum_{t=1}^{n} \epsilon_{t}^{2}$, thus $P\left(\sigma<\widehat{\sigma}_{n}\right)=$ $P\left(\frac{1}{n} \sum_{t=1}^{n} \mathbb{1}_{\epsilon_{t}^{2}=0}>1 / 2\right)=P\left(X_{n}>n / 2\right)$ where $X_{n} \sim \mathcal{B}(n, 1 / 2)$.

We now derive the e.d.f.r. asymptotic distribution under the following additional assumptions.

A6: $\boldsymbol{\theta}_{0}$ belongs to the interior of $\boldsymbol{\Theta}$.

A 7: There exist no non-zero $\boldsymbol{x} \in \mathbb{R}^{d}$ such that $\boldsymbol{x}^{\prime} \frac{\partial \sigma_{t}\left(\boldsymbol{\theta}_{0}\right)}{\partial \boldsymbol{\theta}}=0, \quad$ a.s.

A8: The function $\boldsymbol{\theta} \mapsto \sigma\left(x_{1}, x_{2}, \ldots ; \boldsymbol{\theta}\right)$ has continuous second-order derivatives, and

$$
\sup _{\boldsymbol{\theta} \in \boldsymbol{\Theta}}\left\|\frac{\partial \sigma_{t}(\boldsymbol{\theta})}{\partial \boldsymbol{\theta}}-\frac{\partial \tilde{\sigma}_{t}(\boldsymbol{\theta})}{\partial \boldsymbol{\theta}}\right\| \leq K \rho^{t} .
$$

A9: There exists a neighborhood $V\left(\boldsymbol{\theta}_{0}\right)$ of $\boldsymbol{\theta}_{0}$ such that

$$
E \sup _{\boldsymbol{\theta} \in V\left(\boldsymbol{\theta}_{0}\right)}\left\{\left\|\frac{1}{\sigma_{t}(\boldsymbol{\theta})} \frac{\partial \sigma_{t}(\boldsymbol{\theta})}{\partial \boldsymbol{\theta}}\right\|^{4}+\left\|\frac{1}{\sigma_{t}(\boldsymbol{\theta})} \frac{\partial^{2} \sigma_{t}(\boldsymbol{\theta})}{\partial \boldsymbol{\theta} \partial \boldsymbol{\theta}^{\prime}}\right\|^{2}+\left|\frac{\sigma_{t}\left(\boldsymbol{\theta}_{0}\right)}{\sigma_{t}(\boldsymbol{\theta})}\right|^{4}+\left|\frac{\sigma_{t}(\boldsymbol{\theta})}{\sigma_{t}\left(\boldsymbol{\theta}_{0}\right)}\right|^{4}\right\}<\infty .
$$

Moreover, $\kappa_{4}:=E\left|\eta_{t}\right|^{4}<\infty$.

${ }^{2}$ For the classical $\operatorname{GARCH}(p, q)$ model, Berkes and Horváth (2003) established a Glivenko-Cantelli theorem for the empirical cumulative distribution function (cdf) of the squared residuals. 
A10: All the coordinates of $\frac{\partial \sigma_{t}\left(\boldsymbol{\theta}_{0}\right)}{\partial \boldsymbol{\theta}}$ are (strictly) positive.

A11: $\eta_{1}$ admits a density $f$ which is continuous on $\mathbb{R}$.

A12: For any $\boldsymbol{\theta} \in \Theta$, for any $c>0$, and any sequence $\left(x_{i}\right)$, there exits $\boldsymbol{\theta}_{c} \in \Theta$ such that $c \sigma\left(x_{1}, x_{2}, \ldots ; \boldsymbol{\theta}\right)=\sigma\left(x_{1}, x_{2}, \ldots ; \boldsymbol{\theta}_{c}\right)$.

Assumptions A6 is required for the asymptotic normal distribution of $\widehat{\boldsymbol{\theta}}_{n}$. Assumptions A7-A10 and $\mathbf{A 1 2}$ are satisfied for the standard $\operatorname{GARCH}(p, q)$ model, under the already mentioned regularity assumptions. Assumption A12 is a stability-by-scaling property, which seems a desirable assumption for any volatility model (examples are provided in Francq and Zakoian (2015)).

We now state our first main result showing that the law of $\sqrt{n}\left(\widehat{F}_{n}-F\right)$ is AED but AMF.

Theorem 2. Let A1-A4, A6-A11 hold. Then, for any sequence $\left(x_{n}\right)$ of random variables converging in probability to $x \in \mathbb{R}$,

$$
\sqrt{n}\left(\widehat{F}_{n}\left(x_{n}\right)-F\left(x_{n}\right)\right)=\frac{1}{\sqrt{n}} \sum_{t=1}^{n}\left\{\mathbb{1}_{\eta_{t}<x}-F(x)\right\}-\frac{x f(x)}{2 \sqrt{n}} \boldsymbol{\Omega}^{\prime} \boldsymbol{J}^{-1} \sum_{t=1}^{n}\left(1-\eta_{t}^{2}\right) \boldsymbol{D}_{t}+o_{P}(1)
$$

where $\boldsymbol{\Omega}=E\left(\boldsymbol{D}_{t}\right), \boldsymbol{J}=E\left(\boldsymbol{D}_{t} \boldsymbol{D}_{t}^{\prime}\right)$ with $\boldsymbol{D}_{t}=\boldsymbol{D}_{t}\left(\boldsymbol{\theta}_{0}\right)$.

If in addition $\mathbf{A 1 2}$ holds,

$$
\sqrt{n}\left(\widehat{F}_{n}\left(x_{n}\right)-F\left(x_{n}\right)\right) \stackrel{\mathcal{L}}{\rightarrow} \mathcal{N}\left(0, F(x)\{1-F(x)\}+\frac{\{x f(x)\}^{2}}{4}\left(\kappa_{4}-1\right)+x f(x) \varrho(x)\right)
$$

where $\varrho(x)=E\left(\eta_{0}^{2} \mathbb{1}_{\eta_{0}<x}\right)-F(x)$.

REMARK 1. A noticeable outcome of this theorem is that, under A12, the asymptotic distribution of the e.d.f.r. only depends on the underlying distribution of the innovations. It is completely independent of the model from which these residuals are derived. In the sense of Robinson (1987), it means that the e.d.f.r. is adaptive to the unknown value of $\boldsymbol{\theta}_{0}$, and even to the unknown form of the function $\sigma$. This does not mean that we retrieve the asymptotic distribution of $F_{n}$ derived for iid data. The usual asymptotic variance, $F(x)\{1-F(x)\}$, is only valid when $x f(x)=0$. This is the case when $x=0$ because the innovations and residuals at a given date have the same sign. The effect of estimation also vanishes when $|x|$ increases to $\infty$ (under A9). Parameter estimation may reduce or increase the asymptotic variance (see Appendix).

REMARK 2. One practical interest of adaptiveness is that estimation of the asymptotic variance reduces to estimation of characteristics of the innovations distribution. In particular, $f(x)$ can be estimated by a Kernel density estimator based on the residuals. Theorem 2.1 in Kulperger and Yu (2005) shows that, in the standard GARCH case, the use of residuals instead of innovations has no asymptotic impact on the estimation of the density. 


\section{Including a conditional mean}

In ARMA-GARCH models, the conditional variance specification is completed by a linear model for the conditional mean. In what follows, we consider a more general framework. We will not either restrict our investigations to the QML estimator. Suppose the model writes

$$
\left\{\begin{array}{l}
y_{t}=m_{t}+\epsilon_{t}, \quad \epsilon_{t}=\sigma_{t} \eta_{t} \\
m_{t}=m\left(y_{t-1}, y_{t-2}, \ldots ; \boldsymbol{\theta}_{0}\right), \quad \sigma_{t}=\sigma\left(y_{t-1}, y_{t-2}, \ldots ; \boldsymbol{\theta}_{0}\right)
\end{array}\right.
$$

under the same assumptions on $\left(\eta_{t}\right), \boldsymbol{\theta}_{0}$ and $\boldsymbol{\Theta}$ as before. Model (4) includes the double- $\mathrm{AR}(p)$ of Ling (2007) in which $\boldsymbol{\theta}_{0}=\left(\phi_{01}, \ldots, \phi_{0 p}, \omega_{0}, \alpha_{01}, \ldots, \alpha_{0 p}\right)^{\prime} \in \mathbb{R}^{p} \times(0, \infty) \times[0, \infty)^{p}$ and

$$
m\left(y_{t-1}, y_{t-2}, \ldots ; \boldsymbol{\theta}_{0}\right)=\sum_{i=1}^{p} \phi_{0 i} y_{t-i}, \quad \sigma\left(y_{t-1}, y_{t-2}, \ldots ; \boldsymbol{\theta}_{0}\right)=\sqrt{\omega_{0}+\sum_{i=1}^{p} \alpha_{0 i} y_{t-i}^{2}},
$$

but also more traditional ARMA-GARCH-type models, in which the volatility initially defined in terms of the $\epsilon_{t-i}$ 's can be rewritten as a function of the $y_{t-i}$ 's.

Let, for arbitrary initial values $\tilde{y}_{0}, \tilde{y}_{-1}, \ldots$, for any $\boldsymbol{\theta} \in \mathbf{\Theta}$, and for $1 \leq t \leq n$,

$$
\widetilde{m}_{t}(\boldsymbol{\theta})=m\left(y_{t-1}, y_{t-2}, \ldots, y_{1}, \tilde{y}_{0}, \tilde{y}_{-1}, \ldots ; \boldsymbol{\theta}\right), \quad \tilde{\sigma}_{t}(\boldsymbol{\theta})=\sigma\left(y_{t-1}, y_{t-2}, \ldots, y_{1}, \tilde{y}_{0}, \tilde{y}_{-1}, \ldots ; \boldsymbol{\theta}\right) .
$$

As in the previous section we denote without "tilde", the functions $m_{t}(\boldsymbol{\theta})$ and $\sigma_{t}(\boldsymbol{\theta})$ in which the initial values are replaced by variables at times anterior to 0 . In the following assumptions, $r>0$ denotes a real number which can be chosen arbitrarily small.

B1: $\left(y_{t}\right)$ is a strictly stationary, non-anticipative and ergodic solution of Model (4). Moreover, $E\left|m_{t}\right|^{r}<\infty$ and $E\left|\sigma_{t}\right|^{r}<\infty$.

B2: $\widehat{\boldsymbol{\theta}}_{n}$ denotes any consistent estimator of $\boldsymbol{\theta}_{0}$. The functions $\boldsymbol{\theta} \rightarrow m_{t}(\boldsymbol{\theta})$ and $\boldsymbol{\theta} \rightarrow \sigma_{t}(\boldsymbol{\theta})$ are continuously differentiable. Almost surely, $\sigma_{t}(\boldsymbol{\theta}) \in(\underline{\omega}, \infty]$ for any $\boldsymbol{\theta} \in \boldsymbol{\Theta}$ and for some $\underline{\omega}>0$.

B3: $\sup _{\boldsymbol{\theta} \in \boldsymbol{\Theta}}\left(\left|m_{t}(\boldsymbol{\theta})-\widetilde{m}_{t}(\boldsymbol{\theta})\right|+\left|\sigma_{t}(\boldsymbol{\theta})-\tilde{\sigma}_{t}(\boldsymbol{\theta})\right|\right) \leq K_{t} \rho^{t}$ where $K_{t} \in \mathcal{F}_{t-1}$ and $\sup _{t} E\left(K_{t}^{r}\right)<\infty$.

B4: For any neighborhood $V\left(\boldsymbol{\theta}_{0}\right)$ of $\boldsymbol{\theta}_{0}$, we have

$$
E \sup _{\boldsymbol{\theta} \in V\left(\boldsymbol{\theta}_{0}\right)}\left\{\left\|\frac{\partial m_{t}(\boldsymbol{\theta})}{\partial \boldsymbol{\theta}}\right\|^{r}+\left|m_{t}\left(\boldsymbol{\theta}_{0}\right)-m_{t}(\boldsymbol{\theta})\right|^{r}+\left\|\boldsymbol{D}_{t}(\boldsymbol{\theta})\right\|^{r}\right\}<\infty
$$

where

$$
\boldsymbol{D}_{t}(\boldsymbol{\theta})=\frac{1}{\sigma_{t}(\boldsymbol{\theta})} \frac{\partial \sigma_{t}(\boldsymbol{\theta})}{\partial \boldsymbol{\theta}}
$$

Note that the condition $K \in \mathcal{F}_{0}$ in $\mathbf{A 3}$ has been weaken in B3, in particular to be able to handle the ARMA-GARCH under standard conditions.

We start by extending the Glivenko-Cantelli-type Theorem 1. Let the residuals $\widehat{\eta}_{t}=\left\{y_{t}-\right.$ $\left.\widetilde{m}_{t}\left(\widehat{\boldsymbol{\theta}}_{n}\right)\right\} / \tilde{\sigma}_{t}\left(\widehat{\boldsymbol{\theta}}_{n}\right)$. 
Theorem 3. Under B1-B4 (instead of A1-A4), the conclusions of Theorem 1 hold.

Hence, extending the framework of the previous section does not alter the consistency results. We will now see that the same conclusion cannot be drawn concerning the asymptotic distribution of $\sqrt{n}\left(\widehat{F}_{n}-F\right)$, which will no longer be model-free in general. Another difference, which constitutes a major difficulty, is that Assumption A10 can no longer be made when a conditional mean is included. ${ }^{3}$ We now assume that $\widehat{\boldsymbol{\theta}}_{n}$ admits a Bahadur representation.

B5: The following expansion holds

$$
\sqrt{n}\left(\widehat{\boldsymbol{\theta}}_{n}-\boldsymbol{\theta}_{0}\right)=\frac{1}{\sqrt{n}} \sum_{t=1}^{n} \boldsymbol{\Delta}_{t-1} \boldsymbol{V}\left(\eta_{t}\right)+o_{P}(1)
$$

where $\boldsymbol{V}(\cdot)$ is a measurable function, $\boldsymbol{V}: \mathbb{R} \mapsto \mathbb{R}^{k}$ for some positive integer $k$, and $\boldsymbol{\Delta}_{t-1}$ is a $\mathcal{F}_{t-1}$-measurable $d \times k$ matrix. The variables $\boldsymbol{\Delta}_{t}$ and $\boldsymbol{V}\left(\eta_{t}\right)$ belong to $L^{2}$ with $E \boldsymbol{V}\left(\eta_{t}\right)=$ $0, \quad \operatorname{var}\left\{\boldsymbol{V}\left(\eta_{t}\right)\right\}=\boldsymbol{\Upsilon}$ is nonsingular and $E \boldsymbol{\Delta}_{t}=\boldsymbol{\Lambda}$ is full row rank.

Under this assumption, the CLT for stationary second-order martingale differences of Billingsley (1961) can be applied. It follows that the asymptotic law of $\sqrt{n}\left(\widehat{\boldsymbol{\theta}}_{n}-\boldsymbol{\theta}_{0}\right)$ is a centered Gaussian with variance $\boldsymbol{\Sigma}:=E\left(\boldsymbol{\Delta}_{t} \Upsilon \boldsymbol{\boldsymbol { \Delta } _ { t } ^ { \prime }}\right)$. Assumption B5 has to be verified on a case by case basis, given specific model and estimator.

REMARK 3. For the Gaussian QMLE $\widehat{\boldsymbol{\theta}}_{n}$ of $\boldsymbol{\theta}_{0}$,

$$
\widehat{\boldsymbol{\theta}}_{n}=\arg \min _{\boldsymbol{\theta} \in \boldsymbol{\Theta}} Q_{n}(\boldsymbol{\theta}), \quad Q_{n}(\boldsymbol{\theta})=\frac{1}{n} \sum_{t=1}^{n} \tilde{\ell}_{t}(\boldsymbol{\theta}), \quad \tilde{\ell}_{t}(\boldsymbol{\theta})=\frac{\left\{y_{t}-\tilde{m}_{t}(\boldsymbol{\theta})\right\}^{2}}{\tilde{\sigma}_{t}^{2}(\boldsymbol{\theta})}+\log \tilde{\sigma}_{t}^{2}(\boldsymbol{\theta}),
$$

it can be shown that

$$
\sqrt{n}\left(\widehat{\boldsymbol{\theta}}_{n}-\boldsymbol{\theta}_{0}\right)=2 \boldsymbol{J}^{-1} \frac{1}{\sqrt{n}} \sum_{t=1}^{n}\left\{\eta_{t} \frac{1}{\sigma_{t}} \frac{\partial m_{t}\left(\boldsymbol{\theta}_{0}\right)}{\partial \boldsymbol{\theta}}+\left(\eta_{t}^{2}-1\right) \frac{1}{\sigma_{t}} \frac{\partial \sigma_{t}\left(\boldsymbol{\theta}_{0}\right)}{\partial \boldsymbol{\theta}}\right\}+o_{P}(1),
$$

where

$$
\boldsymbol{J}=E\left(\frac{\partial \ell_{t}^{2}\left(\boldsymbol{\theta}_{0}\right)}{\partial \boldsymbol{\theta} \partial \boldsymbol{\theta}^{\prime}}\right)=2 \boldsymbol{J}_{m}+4 \boldsymbol{J}_{\sigma}, \quad \boldsymbol{J}_{m}=E\left(\frac{1}{\sigma_{t}^{2}} \frac{\partial m_{t}\left(\boldsymbol{\theta}_{0}\right)}{\partial \boldsymbol{\theta}} \frac{\partial m_{t}\left(\boldsymbol{\theta}_{0}\right)}{\partial \boldsymbol{\theta}^{\prime}}\right)
$$

and $\boldsymbol{J}_{\sigma}=E \boldsymbol{D}_{t}\left(\boldsymbol{\theta}_{0}\right) \boldsymbol{D}_{t}^{\prime}\left(\boldsymbol{\theta}_{0}\right)$. The Bahadur expansion in $\mathbf{B 5}$ is thus satisfied, if $E\left(\eta_{t}\right)=0$, with $k=2$ and

$$
\boldsymbol{V}\left(\eta_{t}\right)=\left(\eta_{t}, \eta_{t}^{2}-1\right)^{\prime}, \quad \boldsymbol{\Delta}_{t-1}=2 \boldsymbol{J}^{-1}\left[\frac{1}{\sigma_{t}} \frac{\partial m_{t}\left(\boldsymbol{\theta}_{0}\right)}{\partial \boldsymbol{\theta}} \quad \frac{1}{\sigma_{t}} \frac{\partial \sigma_{t}\left(\boldsymbol{\theta}_{0}\right)}{\partial \boldsymbol{\theta}}\right], \quad \boldsymbol{\Lambda}=2 \boldsymbol{J}^{-1}\left[\begin{array}{ll}
\boldsymbol{\Omega}_{m} & \boldsymbol{\Omega}_{\sigma}
\end{array}\right] .
$$

We also have $\operatorname{Var}_{a s}\left\{\sqrt{n}\left(\widehat{\boldsymbol{\theta}}_{n}-\boldsymbol{\theta}_{0}\right)\right\}=4 \boldsymbol{J}^{-1}\left\{\boldsymbol{J}_{m}+\left(\kappa_{4}-1\right) \boldsymbol{J}_{\sigma}+\mu_{3}\left(\boldsymbol{J}_{m \sigma}+\boldsymbol{J}_{m \sigma}^{\prime}\right)\right\} \boldsymbol{J}^{-1}=\boldsymbol{\Sigma}$, where $\boldsymbol{J}_{m \sigma}=E\left(\frac{1}{\sigma_{t}^{2}} \frac{\partial m_{t}\left(\boldsymbol{\theta}_{0}\right)}{\partial \boldsymbol{\theta}} \frac{\partial \sigma_{t}\left(\boldsymbol{\theta}_{0}\right)}{\partial \boldsymbol{\theta}^{\prime}}\right)$ and $\mu_{3}=E \eta_{t}^{3}$.

${ }^{3}$ For instance in the model $y_{t}=m_{0}+\sigma_{t} \eta_{t}$ where $\sigma_{t}^{2}=\omega+\alpha\left(y_{t-1}-m\right)^{2}$, the derivative of $\sigma_{t}$ with respect to $m$ is not positive, and even not of constant sign. 
The assumptions of Section 2 have to be modified as follows.

B6: The function $\boldsymbol{\theta} \rightarrow m_{t}(\boldsymbol{\theta})$ and $\boldsymbol{\theta} \rightarrow \sigma_{t}(\boldsymbol{\theta})$ have continuous second-order derivatives, and

$$
\sup _{\boldsymbol{\theta} \in \boldsymbol{\Theta}}\left\|\frac{\partial m_{t}(\boldsymbol{\theta})}{\partial \boldsymbol{\theta}}-\frac{\partial \widetilde{m}_{t}(\boldsymbol{\theta})}{\partial \boldsymbol{\theta}}\right\|+\sup _{\boldsymbol{\theta} \in \boldsymbol{\Theta}}\left\|\frac{\partial \sigma_{t}(\boldsymbol{\theta})}{\partial \boldsymbol{\theta}}-\frac{\partial \widetilde{\sigma}_{t}(\boldsymbol{\theta})}{\partial \boldsymbol{\theta}}\right\| \leq K_{t} \rho^{t},
$$

where $K_{t}$ is as in $\mathbf{B 3}$.

B7: There exists a neighborhood $V\left(\boldsymbol{\theta}_{0}\right)$ of $\boldsymbol{\theta}_{0}$ such that

$$
E \sup _{\boldsymbol{\theta} \in V\left(\boldsymbol{\theta}_{0}\right)}\left\{\left\|\frac{1}{\sigma_{t}\left(\boldsymbol{\theta}_{0}\right)} \frac{\partial m_{t}(\boldsymbol{\theta})}{\partial \boldsymbol{\theta}}\right\|^{4}+\left\|\frac{1}{\sigma_{t}\left(\boldsymbol{\theta}_{0}\right)} \frac{\partial^{2} m_{t}(\boldsymbol{\theta})}{\partial \boldsymbol{\theta} \partial \boldsymbol{\theta}^{\prime}}\right\|^{2}\right\}<\infty .
$$

Let the additional assumption

B8: For $n$ large enough, $n>n_{0}$ say, the conditional distribution of $\eta_{t}$ given $\widehat{\boldsymbol{\theta}}_{n}$ and $\mathcal{F}_{t-1}$ admits a density which is a.s. bounded, uniformly in $n>n_{0}$ and $t<n-t(n)$ where $t(n)=o(\sqrt{n})$.

Intuitively, the variables $\widehat{\boldsymbol{\theta}}_{n}$ and $\eta_{t}$ should be asymptotically independent in most standard situations. For instance, consider the simple location model $y_{t}=\theta_{0}+\sigma \eta_{t}$, with Gaussian innovations $\eta_{t}$. The conditional distribution of $\eta_{t}$ given $\widehat{\theta}_{n}$ and $\mathcal{F}_{t-1}$, where $\widehat{\theta}_{n}$ is the sample mean, is Gaussian with variance $1-\{n-(t-1)\}^{-1}$. Thus $\mathbf{B} 8$ is satisfied for $n_{0}>1$ and $t(n)=1$, with bound $1 / \sqrt{\pi}$.

The next theorem uses the notion of discrete estimator, which has been introduced by Le Cam (1960) and used by many authors (e.g. Kreiss (1987)).

Theorem 4. Under B1-B8 and A9, A11, if i) $\left(x_{n}\right)$ is a non-random sequence converging to $x \in \mathbb{R}$; or ii) $x_{n}$ is a discrete $\sqrt{n}$-consistent estimator of $x$, then

$$
\sqrt{n}\left(\widehat{F}_{n}\left(x_{n}\right)-F\left(x_{n}\right)\right) \stackrel{\mathcal{L}}{\rightarrow} \mathcal{N}\left(0, F(x)\{1-F(x)\}+\boldsymbol{H}^{\prime}(x) \boldsymbol{\Sigma} \boldsymbol{H}(x)+2 \boldsymbol{H}^{\prime}(x) \boldsymbol{\Lambda} \varrho(x)\right)
$$

where $\boldsymbol{\varrho}(x)=E\left(\mathbb{1}_{\eta_{0}<x} \boldsymbol{V}\left(\eta_{0}\right)\right)$ and $\boldsymbol{H}(x)=f(x)\left(\boldsymbol{\Omega}_{m}+x \boldsymbol{\Omega}_{\sigma}\right), \boldsymbol{\Omega}_{m}=E\left(\frac{1}{\sigma_{t}} \frac{\partial m_{t}\left(\boldsymbol{\theta}_{0}\right)}{\partial \boldsymbol{\theta}}\right), \boldsymbol{\Omega}_{\sigma}=E \boldsymbol{D}_{t}\left(\boldsymbol{\theta}_{0}\right)$. Every sequence of $\sqrt{n}$-consistent estimators can be truncated to get a discrete sequence. The discretization can also be avoided, at the price of either reinforcing Assumption B8, or increasing the speed of convergence of the sequence $\left(x_{n}\right)$ (see Appendix).

More explicit forms of the asymptotic variance can be derived for the QMLE under the following extension of Assumption A12 which will be illustrated below.

A12*: For any $\boldsymbol{\theta} \in \Theta$, for any $c>0$, and any sequence $\left(x_{i}\right)$, there exits $\boldsymbol{\theta}_{c} \in \Theta$ such that $c \sigma\left(x_{1}, x_{2}, \ldots ; \boldsymbol{\theta}\right)=\sigma\left(x_{1}, x_{2}, \ldots ; \boldsymbol{\theta}_{c}\right)$ and $m\left(x_{1}, x_{2}, \ldots ; \boldsymbol{\theta}\right)=m\left(x_{1}, x_{2}, \ldots ; \boldsymbol{\theta}_{c}\right)$.

The next result provides an explicit form for the asymptotic variance when the parameters of the conditional mean and variance are independent and are estimated by QML. 
Corollary 1. Let $\boldsymbol{\theta}=\left(\boldsymbol{\varphi}^{\prime}, \boldsymbol{\vartheta}^{\prime}\right)^{\prime}$ and suppose that $m_{t}=m_{t}(\boldsymbol{\varphi}), \sigma_{t}=\sigma_{t}(\boldsymbol{\vartheta})$. Under the assumptions of Theorem 4, under $\mathbf{A} 12^{*}$ and using the QMLE of $\boldsymbol{\theta}_{0}$, we have

$$
\begin{aligned}
& \operatorname{Var}_{a s}\left\{\sqrt{n}\left(\widehat{F}_{n}\left(x_{n}\right)-F\left(x_{n}\right)\right)\right\} \\
= & F(x)\{1-F(x)\}+x f(x)\left\{x f(x) \frac{\kappa_{4}-1}{4}+\varrho(x)\right\}+f(x)\left\{f(x)+2 E\left(\mathbb{1}_{\eta_{0}<x} \eta_{0}\right)\right\} \\
& \times E\left(\frac{1}{\sigma_{t}} \frac{\partial m_{t}\left(\boldsymbol{\varphi}_{0}\right)}{\partial \varphi^{\prime}}\right)\left\{E\left(\frac{1}{\sigma_{t}^{2}} \frac{\partial m_{t}\left(\boldsymbol{\varphi}_{0}\right)}{\partial \boldsymbol{\varphi}} \frac{\partial m_{t}\left(\boldsymbol{\varphi}_{0}\right)}{\partial \varphi^{\prime}}\right)\right\}^{-1} E\left(\frac{1}{\sigma_{t}} \frac{\partial m_{t}\left(\boldsymbol{\varphi}_{0}\right)}{\partial \boldsymbol{\varphi}}\right) .
\end{aligned}
$$

REMARK 4. The latter variance is obviously AMF if $E\left(\frac{1}{\sigma_{t}} \frac{\partial m_{t}\left(\varphi_{0}\right)}{\partial \varphi}\right)=\mathbf{0}$. This occurs in particular for conditionally homoscedastic ARMA models without intercept. ${ }^{4}$ However, this property is not general, as the following example shows. Let

$$
X_{t}=\varphi_{0} \mathbb{1}_{X_{t-1}>0}+\sigma_{0} \eta_{t}, \quad \varphi_{0} \in \mathbb{R}, \quad \sigma_{0}>0,
$$

where, for simplicity, the iid process $\left(\eta_{t}\right)$ is endowed with a symmetric and continuous distribution $F$. Then $\left(X_{t}\right)$ is strictly stationary ${ }^{6}$ and straightforward calculation shows that $P\left(X_{t}>0\right)=$ $\left\{3-2 F\left(\varphi_{0} / \sigma_{0}\right)\right\}^{-1}$. Thus

$$
E\left(\frac{1}{\sigma_{0}} \frac{\partial m_{t}\left(\boldsymbol{\varphi}_{0}\right)}{\partial \boldsymbol{\varphi}^{\prime}}\right)\left\{E\left(\frac{1}{\sigma_{0}^{2}} \frac{\partial m_{t}\left(\boldsymbol{\varphi}_{0}\right)}{\partial \boldsymbol{\varphi}} \frac{\partial m_{t}\left(\boldsymbol{\varphi}_{0}\right)}{\partial \boldsymbol{\varphi}^{\prime}}\right)\right\}^{-1} E\left(\frac{1}{\sigma_{0}} \frac{\partial m_{t}\left(\boldsymbol{\varphi}_{0}\right)}{\partial \boldsymbol{\varphi}}\right)=\frac{1}{3-2 F\left(\varphi_{0} / \sigma_{0}\right)} .
$$

For this model, the distribution of the e.d.f.r. is not $A M F$.

REMARK 5. When $\mathbf{A 1 2 *}$ does not hold, even when the conditional mean and variance parameters are disentangled, the variance is AMD, as the following example shows. Consider the following DAR(1) model

$$
X_{t}=\phi_{0} X_{t-1}+\sqrt{1+\alpha_{0} X_{t-1}^{2}} \eta_{t}, \quad \alpha_{0}>0, \quad \phi_{0} \in \mathbb{R}, \quad \boldsymbol{\theta}=(\phi, \alpha)^{\prime},
$$

under the strict stationarity condition $E \log \left|\phi_{0}+\sqrt{\alpha_{0}} \eta_{t}\right|<0$ (see Ling, 2007). Then it can be shown that the asymptotic variance of $\sqrt{n}\left(\widehat{F}_{n}\left(x_{n}\right)-F\left(x_{n}\right)\right)$ depends on the value of $\boldsymbol{\theta}_{0}$ (see Appendix).

${ }^{4}$ Let $\Phi(L) y_{t}=\Theta(L) \epsilon_{t}$ under standard assumptions on the lag polynomials $\Phi(L)=1-\sum_{i=1}^{p} \phi_{0 i} L^{i}$ and $\Theta(L)=1-\sum_{j=1}^{q} \theta_{0 j} L^{j}$. Then $m_{t}=\sum_{i=1}^{p} \phi_{i} y_{t-i}-\sum_{j=1}^{q} \theta_{j} \epsilon_{t-j}$, from which we deduce $\frac{\partial m_{t}\left(\boldsymbol{\varphi}_{0}\right)}{\partial \phi_{i}}=y_{t-i}-$ $\sum_{j=1}^{q} \theta_{0 j} \frac{\partial \epsilon_{t-j}}{\partial \phi_{i}}$, and $\frac{\partial m_{t}\left(\boldsymbol{\varphi}_{0}\right)}{\partial \theta_{k}}=-\sum_{j=1}^{q} \theta_{0 j} \frac{\partial \epsilon_{t-j}}{\partial \theta_{k}}-\epsilon_{t-k}$. We also have $\frac{\partial \epsilon_{t}}{\partial \theta_{k}}=\Theta(L)^{-1} \epsilon_{t-k}$, hence $E\left(\frac{\partial \epsilon_{t}}{\partial \theta_{k}}\right)=0$ and similarly $E\left(\frac{\partial \epsilon_{t}}{\partial \phi_{i}}\right)=0$. We conclude that $E\left(\frac{1}{\sigma_{0}} \frac{\partial m_{t}\left(\boldsymbol{\varphi}_{0}\right)}{\partial \varphi}\right)$.

${ }^{5}$ This can be shown by noting that $\left(X_{t}\right)$ is a Markov chain for which the ergodicity criterion of Feigin and Tweedie (1985, Theorem 1) applies. Indeed, for $V(x)=|x|+1$ and $M>\left|\varphi_{0}\right|+\sigma_{0} E\left|\eta_{t}\right|$, we have $E\left\{V\left(X_{t}\right) \mid X_{t-1}\right\} \leq\left|\varphi_{0}\right|+\sigma_{0} E\left|\eta_{t}\right|+1 \leq(1-\delta) V(x)$ for $|x|>M$ and $\delta$ sufficiently small.

${ }^{6}$ This can also be shown by noting that $Z_{t}:=\mathbf{1}_{X_{t}>0}=a_{t} Z_{t-1}+b_{t}\left(1-Z_{t-1}\right)$ where $a_{t}=\mathbf{1}_{\varphi_{0}+\sigma_{0} \eta_{t}>0}, b_{t}=$ $\mathbf{1}_{\sigma_{0} \eta_{t}>0}$. Thus $Z_{t}=b_{t}+\sum_{k=0}^{\infty} c_{t} \ldots c_{t-k} b_{t-k-1}$ a.s. where $c_{t}=a_{t}-b_{t}$, where the existence of the infinite sum holds by absolute convergence of the partial sums. 
We now consider in details the case of the $\operatorname{ARMA}(P, Q)-\operatorname{GARCH}(p, q)$ model estimated by Gaussian QML, which constitutes the model and estimation method most widely used in empirical works. Let

$$
\Phi(L) X_{t}=\Theta(L) \epsilon_{t}, \quad \epsilon_{t}=\sigma_{t} \eta_{t}, \quad \sigma_{t}^{2}=\omega+\sum_{i=1}^{q} \alpha_{i} \epsilon_{t-i}^{2}+\sum_{j=1}^{p} \beta_{j} \sigma_{t-j}^{2},
$$

with $\omega>0, \alpha_{i}, \beta_{j} \geq 0$ and under standard assumptions on the lag polynomials $\Phi(L)=$ $1-\sum_{i=1}^{P} \phi_{i} L^{i}$ and $\Psi(L)=1-\sum_{j=1}^{Q} \psi_{j} L^{j}$. Note that this model does not satisfy the assumptions of Corollary 1 because the conditional variance function depends on the whole set of parameters. However, denoting by $\varphi$ the ARMA parameters, Assumption A12* is satisfied, with $\boldsymbol{\theta}_{c}=\left(\boldsymbol{\varphi}^{\prime}, c^{2} \omega, c^{2} \alpha_{1}, \ldots, c^{2} \alpha_{q}, \beta_{1}, \ldots, \beta_{p}\right)^{\prime}$, which entails a considerable simplification (see Appendix).

Corollary 2. For the ARMA-GARCH model (6) with symmetrically distributed innovations, under the assumptions ensuring the CAN of the QMLE, under $\mathbf{B} 8$ and $\mathbf{A 1 1}$, and for the sequence $\left(x_{n}\right)$ of Theorem 4, we have

$$
\begin{aligned}
& \sqrt{n}\left(\widehat{F}_{n}\left(x_{n}\right)-F\left(x_{n}\right)\right) \\
\stackrel{\mathcal{L}}{\rightarrow} & \mathcal{N}\left(0, F(x)\{1-F(x)\}+\frac{\{x f(x)\}^{2}}{4}\left(\kappa_{4}-1\right)+x f(x) E\left\{\mathbb{1}_{\eta_{0}<x}\left(\eta_{0}^{2}-1\right)\right\}\right) .
\end{aligned}
$$

In the symmetric ARMA-GARCH case, the asymptotic distribution of the empirical cdf of the residuals is thus adaptive to the parametric specification contrary to the examples of Remarks 4 and 5. In particular, in the pure ARMA case, estimating the (constant) innovations variance suffices to introduce estimation dependence in the asymptotic variance (compare to Bai (1994), Theorem 1).

We now exploit our stochastic equicontinuity results for estimating conditional quantiles.

\section{Estimation of the conditional VaR}

The conditional VaR of the process $\left(\epsilon_{t}\right)$ at risk level $\alpha \in(0,1)$, denoted by $\operatorname{VaR}_{t}(\alpha)$, is defined as the opposite of the $\alpha$-quantile of the conditional distribution of $\epsilon_{t}$. Assuming that this distribution is continuous, $\operatorname{VaR}_{t}(\alpha)$ solves

$$
P_{t-1}\left[\epsilon_{t}<-\operatorname{VaR}_{t}(\alpha)\right]=\alpha
$$

where $P_{t-1}$ denotes the historical distribution conditional on $\left\{\epsilon_{u}, u<t\right\}$. When $\left(\epsilon_{t}\right)$ is a nonanticipative solution of Model (1), the conditional VaR at level $\alpha$ is then given by

$$
\operatorname{VaR}_{t}(\alpha)=-\sigma\left(\epsilon_{t-1}, \epsilon_{t-2}, \ldots ; \boldsymbol{\theta}_{0}\right) \xi_{\alpha}
$$

where $\xi_{\alpha}=\inf \{x: F(x) \geq \alpha\}$ is the $\alpha$-quantile of the cdf $F$ of $\eta_{t}$. A two-step estimator of the conditional VaR at level $\alpha$ is thus $\widehat{\operatorname{VaR}}_{t}(\alpha)=-\tilde{\sigma}\left(\epsilon_{t-1}, \epsilon_{t-2}, \ldots ; \widehat{\boldsymbol{\theta}}_{n}\right) \widehat{\xi}_{n, \alpha}$, where $\widehat{\xi}_{n, \alpha}$ is the 
$\alpha$-quantile of $\widehat{\eta}_{1}, \ldots, \widehat{\eta}_{n}$, that is the $\lceil n \alpha\rceil$-th order statistics of the residuals, where $\lceil x\rceil$ denotes the smallest integer larger than $x$. For standard GARCH, Asymmetric Power GARCH and DAR models, alternative estimators based on the quantile equivariance property and quantile regression have been recently studied by Zheng, Zhu, Li and Xiao (2018), Wang, Zhu, Li and Li (2019), and Zhu and Li (2019). For ARMA-GARCH models, estimators of $\xi_{\alpha}$ based on extreme value theory were investigated by Hoga (2019).

We start by establishing the strong consistency of the empirical quantiles of the residuals. We make the following assumption.

A13: For $\alpha \in(0,1)$, the cdf $F$ of $\eta$ satisfies: $F(x)>\alpha \quad$ whenever $\quad x>\xi_{\alpha}$.

Assumption $\mathbf{A 1 3}$ means that the quantile function $F^{-}$of $\eta$ is right-continuous at $\alpha$.

Corollary 3. For Model (1) under A1-A5, A13, for $\alpha \in(0,1)$ we have the strong convergence

$$
\widehat{\xi}_{n, \alpha} \rightarrow \xi_{\alpha} \quad \text { a.s. }
$$

Without Assumption A13, we have

$$
\left[\liminf \widehat{\xi}_{n, \alpha}, \lim \sup \widehat{\xi}_{n, \alpha}\right] \subseteq\left[\xi_{\alpha}, \xi_{\alpha}^{+}\right] \quad \text { a.s. }
$$

where $\xi_{\alpha}^{+}=\inf \{x: F(x)>\alpha\}$.

When the residuals $\widehat{\eta}_{t}$ are replaced by innovations $\eta_{t}$ (and $\widehat{\xi}_{n, \alpha}$ replaced by $\xi_{n, \alpha}$ ) the inclusion in (7) is in fact an equality (see Appendix). In particular, the empirical quantile $\xi_{n, \alpha}$ of the innovations, and in general the empirical quantile $\widehat{\xi}_{n, \alpha}$ of the residuals, do not converge when Assumption A13 is not satisfied.

To establish the asymptotic distribution of $\widehat{\xi}_{n, \alpha}$, we need the following assumption.

A14: the density $f$ is strictly positive in a neighborhood of $\xi_{\alpha}$.

Corollary 4. For Model (1), under A1-A4, A6-A12 and A14, we have

$$
\sqrt{n}\left(\widehat{\xi}_{n, \alpha}-\xi_{\alpha}\right)=-\frac{1}{f\left(\xi_{\alpha}\right)} \frac{1}{\sqrt{n}} \sum_{t=1}^{n}\left(\mathbf{1}_{\left\{\eta_{t}<\xi_{\alpha}\right\}}-\alpha\right)+\xi_{\alpha} \boldsymbol{\Omega}^{\prime} \frac{\boldsymbol{J}^{-1}}{2 \sqrt{n}} \sum_{t=1}^{n}\left(1-\eta_{t}^{2}\right) \boldsymbol{D}_{t}+o_{P}(1) .
$$

Thus

$$
\sqrt{n}\left(\widehat{\xi}_{n, \alpha}-\xi_{\alpha}\right) \stackrel{\mathcal{L}}{\rightarrow} \mathcal{N}\left(0, \frac{\alpha(1-\alpha)}{f^{2}\left(\xi_{\alpha}\right)}+\frac{\xi_{\alpha} \varrho\left(\xi_{\alpha}\right)}{f\left(\xi_{\alpha}\right)}+\frac{\kappa_{4}-1}{4} \xi_{\alpha}^{2}\right) .
$$

Direct proof of this result would be quite complex, but with the help of Theorem 2 it is simplistic. 
Proof. Note that Assumptions A5 and A13 are satisfied under A11 and A14. Thus, by Corollary $3, \widehat{\xi}_{n, \alpha} \rightarrow \xi_{\alpha} \quad$ a.s. The empirical cdf being a step function with jumps of size $1 / n$, we have $\widehat{F}_{n}\left(\widehat{\xi}_{n, \alpha}\right)-$ $\alpha \leq 1 / n$. It follows that, by Theorem 2 ,

$$
\begin{aligned}
& \sqrt{n}\left(\alpha-F\left(\widehat{\xi}_{n, \alpha}\right)\right)=\sqrt{n}\left(\widehat{F}_{n}\left(\widehat{\xi}_{n, \alpha}\right)-F\left(\widehat{\xi}_{n, \alpha}\right)\right)+o_{P}(1) \\
= & \frac{1}{\sqrt{n}} \sum_{t=1}^{n}\left\{\mathbb{1}_{\eta_{t}<\xi_{\alpha}}-F\left(\xi_{\alpha}\right)\right\}-\frac{\xi_{\alpha} f\left(\xi_{\alpha}\right)}{2 \sqrt{n}} \boldsymbol{\Omega}^{\prime} \boldsymbol{J}^{-1} \sum_{t=1}^{n}\left(1-\eta_{t}^{2}\right) \boldsymbol{D}_{t}+o_{P}(1) .
\end{aligned}
$$

By the delta method applied with the function $F^{-1}$ we conclude.

Again, we emphasize the fact that the distribution of the empirical quantile of the residuals is AMF, though not AEF (since the second and third terms in the asymptotic variance vanish when the residuals are replaced by the innovations). Asymptotic confidence intervals for the conditional VaR could be derived from the joint asymptotic distribution of $\widehat{\boldsymbol{\theta}}_{n}$ and $\widehat{\xi}_{n, \alpha}$ as in Theorem 4 of Francq and Zakoian (2015).

\section{Concluding remarks}

The main contribution of this article is to show that the asymptotic distribution of the residuals of conditional location-scale models can be model free. In the absence of a conditional mean, the effect of estimation on the asymptotic variance is dependent of the error distribution but does not depend on the parametric model. When the conditional mean is present in the model, we give explicit characterizations of situations where this unexpected adaptiveness property holds, leading to simple-to-implement tests on the error distribution.

We now point out several topics for future research. Multivariate extensions are far from trivial because the quantile function does not have a natural extension (see Hallin, Paindaveine and Šiman (2010) for a new multivariate concept of quantile). However, when the innovations have a spherical distribution, estimating the quantile of a linear combination reduces to estimating the cdf of any component of the innovation vector. It would be interesting to extend the results of our paper in this setting (in particular to handle applications involving the VaR of portfolios). Another area of extension concerns nonstationary time series. Ling (1998) showed that the inclusion of a unit root in autoregressive processes renders AMD the distribution of the e.d.f.r., unlike in the stationary case. In the case of an explosive GARCH component, the intercept cannot be consistently estimated (see Francq and Zakoian (2013b)) and the consistency of the cdf of the residuals is an open question. 


\section{Proofs}

This section contains the proofs of Theorems 1,2, 4. More detailed proofs can be found in the Appendix.

\subsection{Proof of Theorem 1}

Let $\eta_{t}(\boldsymbol{\theta})=\epsilon_{t} / \sigma_{t}(\boldsymbol{\theta})$ and $\tilde{\eta}_{t}(\boldsymbol{\theta})=\epsilon_{t} / \tilde{\sigma}_{t}(\boldsymbol{\theta})$, so that $\eta_{t}=\eta_{t}\left(\boldsymbol{\theta}_{0}\right)$ and $\widehat{\eta}_{t}=\tilde{\eta}_{t}\left(\widehat{\boldsymbol{\theta}}_{n}\right)$. By A2-A3,

$$
\sup _{\boldsymbol{\theta} \in \boldsymbol{\Theta}}\left|\eta_{t}(\boldsymbol{\theta})-\tilde{\eta}_{t}(\boldsymbol{\theta})\right| \leq K \rho^{t}\left|\epsilon_{t}\right| .
$$

Moreover, a Taylor expansion shows that

$$
\eta_{t}\left(\widehat{\boldsymbol{\theta}}_{n}\right)=\eta_{t}-\eta_{t}\left(\boldsymbol{\theta}_{t}\right) \boldsymbol{D}_{t}^{\prime}\left(\boldsymbol{\theta}_{t}\right)\left(\widehat{\boldsymbol{\theta}}_{n}-\boldsymbol{\theta}_{0}\right)
$$

where $\boldsymbol{\theta}_{t}$ is between $\widehat{\boldsymbol{\theta}}_{n}$ and $\boldsymbol{\theta}_{0}$. Under A1-A3, $\widehat{\boldsymbol{\theta}}_{n}$ tends to $\boldsymbol{\theta}_{0}$ a.s. (see Francq and Zakoian (2013a), Theorem 1). Since $\widehat{\eta}_{t}=\tilde{\eta}_{t}\left(\widehat{\boldsymbol{\theta}}_{n}\right)$, it follows that

$$
\left|\widehat{\eta}_{t}-\eta_{t}\right| \leq\left|\eta_{t}\right| \sup _{\boldsymbol{\theta} \in V\left(\boldsymbol{\theta}_{0}\right)} \frac{\sigma_{t}\left(\boldsymbol{\theta}_{0}\right)}{\sigma_{t}(\boldsymbol{\theta})} \sup _{\boldsymbol{\theta} \in V\left(\boldsymbol{\theta}_{0}\right)}\left\|\boldsymbol{D}_{t}(\boldsymbol{\theta})\right\|\left\|\widehat{\boldsymbol{\theta}}_{n}-\boldsymbol{\theta}_{0}\right\|+K \rho^{t}\left|\epsilon_{t}\right|
$$

for $n$ large enough. Thus, we have

$$
\left|\widehat{\eta}_{t}-\eta_{t}\right| \leq K\left(\rho^{t} \sigma_{t}+\left\|\widehat{\boldsymbol{\theta}}_{n}-\boldsymbol{\theta}_{0}\right\|\right) u_{t}\left|\eta_{t}\right|
$$

for $n$ large enough, where $u_{t} \in \mathcal{F}_{t-1}$ and, by A1, A2, A4, $E\left(\left|u_{t}\right|^{r / 2}\right)<\infty$. Without loss of generality, assume that $K=1$ in (9). Let $a_{n}=\left\|\widehat{\boldsymbol{\theta}}_{n}-\boldsymbol{\theta}_{0}\right\|$. For all $x \in \mathbb{R}, \varepsilon>0, S>0$ and $M>0$, we then have

$$
\begin{aligned}
\left|1_{\left\{\hat{\eta}_{t} \leq x\right\}}-1_{\left\{\eta_{t} \leq x\right\}}\right| & \leq 1_{\left\{x-\left(\rho^{t} \sigma_{t}+a_{n}\right) u_{t}\left|\eta_{t}\right| \leq \eta_{t} \leq x+\left(\rho^{t} \sigma_{t}+a_{n}\right) u_{t}\left|\eta_{t}\right|\right\}} \\
& \leq 1_{A_{t, \varepsilon, M, S}}+1_{a_{n}>\varepsilon}+1_{u_{t}\left|\eta_{t}\right|>M}+1_{\sigma_{t}>S},
\end{aligned}
$$

with the event $A_{t, \varepsilon, M, S}=\left\{x-\left(\rho^{t} S+\varepsilon\right) M \leq \eta_{t} \leq x+\left(\rho^{t} S+\varepsilon\right) M\right\}$. For $t$ large enough such that $\rho^{t} S \leq \varepsilon$, we have $A_{t, \varepsilon, M, S} \subset A_{t, 2 \varepsilon, M}$ with $A_{t, \varepsilon, M}=\left\{x-\varepsilon M \leq \eta_{t} \leq x+\varepsilon M\right\}$. Assumption A5(x) implies, for $\varepsilon M$ small enough, $E 1_{A_{t, \varepsilon, M}}=\int_{x-\varepsilon M}^{x+\varepsilon M} d F(y) \leq 2 K_{0} \varepsilon M$ where $K_{0}$ is the Lipschitz constant. For all $\kappa>0$, we thus have a small $\varepsilon>0$ and large $M>0$ and $S>0$ such that $E\left\{1_{A_{t, 2 \varepsilon, M}}+1_{u_{t}\left|\eta_{t}\right|>M}+1_{\sigma_{t}>S}\right\} \leq \kappa$. Recall that, under A1-A3, $a_{n}$ tends to zero almost surely, and thus $1_{a_{n}>\varepsilon}=0$ for $n$ large enough. Since $\kappa$ can be chosen arbitrarily small, it follows that, almost surely, by the ergodic theorem we have

$$
\lim _{n \rightarrow \infty} \frac{1}{n} \sum_{t=1}^{n} 1_{\left\{\hat{\eta}_{t} \leq x\right\}}=\lim _{n \rightarrow \infty} \frac{1}{n} \sum_{t=1}^{n} 1_{\left\{\eta_{t} \leq x\right\}}=P\left(\eta_{t} \leq x\right), \quad \forall x \in \mathbb{R} .
$$

We have shown that $\left|\widehat{F}_{n}(x)-F(x)\right| \rightarrow 0$ a.s. and the uniform convergence follows from the fact that, for any $\varepsilon>0$, any cdf has a finite number of jumps of size larger than $\varepsilon$. 


\subsection{A Marcinkiewicz type law of large numbers}

The following result, which will be used in the proof of Theorem 2 is of independent interest. Since we have not been able to find this result in the literature, we provide a proof, which is adapted from arguments kindly given to us by L. Horváth.

Lemma 1. Let $\left(X_{t}, \mathcal{F}_{t}^{X}\right)$ a strictly stationary martingale difference sequence, where $\mathcal{F}_{t}^{X}$ is a filtration, such that $E X_{t}^{2}<\infty$. Then, for any $s>0, \quad \frac{1}{n^{1 / 2+s}} \sum_{t=1}^{n} X_{t} \rightarrow 0$ a.s.

Proof. Let $S_{n}=\sum_{t=1}^{n} X_{t}$. We have, using the martingale difference property,

$$
E S_{n}^{2}=n E X_{1}^{2}
$$

In view of Theorem 15.1 in Burkholder (1973), we have the Rosenthal's type inequality

$$
E \max _{1 \leq k \leq n} S_{k}^{2} \leq K n
$$

Letting $\varsigma>0$, using (10) and the Markov inequality, we have

$$
P\left(\left|S_{\left\lfloor k^{\varsigma}\right\rfloor}\right| \geq \varepsilon\left\lfloor k^{\varsigma}\right\rfloor^{s+1 / 2}\right) \leq \frac{\left.E S_{\lfloor k \varsigma}^{2}\right\rfloor}{\varepsilon^{2}\left\lfloor k^{\varsigma}\right\rfloor^{2(s+1 / 2)}} \leq \frac{K\left\lfloor k^{\varsigma}\right\rfloor}{\varepsilon^{2}\left\lfloor k^{\varsigma}\right\rfloor^{2(s+1 / 2)}}=\frac{K}{\varepsilon^{2}\left\lfloor k^{\varsigma}\right\rfloor^{2 s}},
$$

which is summable provided $\varsigma>1 / 2 s$. It follows, by the Borel-Cantelli lemma, that

$$
\frac{S_{\left\lfloor k^{\varsigma}\right\rfloor}}{\left(\left\lfloor k^{\varsigma}\right\rfloor\right)^{s+1 / 2}} \rightarrow 0, \quad \text { a.s } \quad \text { as } k \rightarrow \infty \text { when } \varsigma>1 / 2 s .
$$

When $n \in\left[k^{\varsigma},(k+1)^{\varsigma}\right]$, we write $S_{n}=S_{\left\lfloor k^{\varsigma}\right\rfloor}+\sum_{i=\left\lfloor k^{\varsigma}\right\rfloor+1}^{n} X_{i}$. Using (11) and noting that the length of the interval $\left[k^{\varsigma},(k+1)^{\varsigma}\right]$ is less than $\varsigma(k+1)^{\varsigma-1}$ when $\varsigma>1$, we have

$$
P\left(\max _{\left\lfloor k^{\varsigma}\right\rfloor \leq j \leq\left\lfloor(k+1)^{\varsigma}\right\rfloor}\left|\sum_{i=\left\lfloor k^{\varsigma}\right\rfloor+1}^{j} X_{i}\right| \geq \varepsilon k^{\varsigma(s+1 / 2)}\right) \leq \frac{K}{\varepsilon^{2} k^{2 s \varsigma+1}},
$$

which is always summable. The result follows by

$$
\frac{\left|S_{n}\right|}{n^{s+1 / 2}} \leq \frac{\left|S_{\left\lfloor k^{\varsigma}\right\rfloor}\right|}{\left(\left\lfloor k^{\varsigma}\right\rfloor\right)^{s+1 / 2}}+\frac{\max _{\left\lfloor k^{\varsigma}\right\rfloor \leq j \leq\left\lfloor(k+1)^{\varsigma}\right\rfloor}\left|\sum_{i=\left\lfloor k^{\varsigma}\right\rfloor+1}^{j} X_{i}\right|}{k^{\varsigma(s+1 / 2)}} .
$$

\subsection{Proof of Theorem 2}

Recall that $F_{n}(x)=\frac{1}{n} \sum_{t=1}^{n} \mathbb{1}_{\left\{\eta_{t} \leq x\right\}}$ and let

$$
\begin{aligned}
\widehat{e}_{n}(x) & =\sqrt{n}\left\{\widehat{F}_{n}(x)-F(x)\right\}, \quad e_{n}(x)=\sqrt{n}\left\{F_{n}(x)-F(x)\right\}, \quad \tilde{\chi}_{t, n}=\tilde{\sigma}_{t}\left(\widehat{\boldsymbol{\theta}}_{n}\right) / \sigma_{t}\left(\boldsymbol{\theta}_{0}\right), \\
\chi_{t, n} & =\sigma_{t}\left(\widehat{\boldsymbol{\theta}}_{n}\right) / \sigma_{t}\left(\boldsymbol{\theta}_{0}\right), \quad h_{n}(x)=x f(x)\left\{\frac{1}{n} \sum_{t=1}^{n} \boldsymbol{D}_{t}^{\prime}\left(\boldsymbol{\theta}_{0}\right)\right\} \sqrt{n}\left(\widehat{\boldsymbol{\theta}}_{n}-\boldsymbol{\theta}_{0}\right) .
\end{aligned}
$$


We have $\widehat{e}_{n}(x)$

$$
=\underbrace{\frac{1}{\sqrt{n}} \sum_{t=1}^{n} \mathbb{1}_{\eta_{t} \leq x \chi_{t, n}}-F\left(x \chi_{t, n}\right)}_{\widehat{e}_{n, 1}(x)}+\underbrace{\frac{1}{\sqrt{n}} \sum_{t=1}^{n} F\left(x \chi_{t, n}\right)-F(x)}_{\widehat{e}_{n, 2}(x)}+\underbrace{\frac{1}{\sqrt{n}} \sum_{t=1}^{n} \mathbb{1}_{\eta_{t} \leq x \tilde{\chi}_{t, n}}-\mathbb{1}_{\eta_{t} \leq x \chi_{t, n}}}_{\widehat{e}_{n, 3}(x)} .
$$

Let, for $\boldsymbol{a}$ a vector of the same size as $\boldsymbol{\theta}$ (sufficiently small so that $\boldsymbol{\theta}_{0}+\boldsymbol{a} / \sqrt{n} \in \mathbf{\Theta}$ ),

$$
e_{n, 1}(x, \boldsymbol{a})=\frac{1}{\sqrt{n}} \sum_{t=1}^{n}\left\{\mathbb{1}_{\eta_{t} \leq x \gamma_{t, n}(\boldsymbol{a})}-F\left(x \gamma_{t, n}(\boldsymbol{a})\right)\right\}, \quad \gamma_{t, n}(\boldsymbol{a})=\frac{\sigma_{t}\left(\boldsymbol{\theta}_{0}+\frac{\boldsymbol{a}}{\sqrt{n}}\right)}{\sigma_{t}\left(\boldsymbol{\theta}_{0}\right)} .
$$

Write

$$
e_{n, 1}(x, \boldsymbol{a})-e_{n}(x)=\frac{1}{\sqrt{n}} \sum_{t=1}^{n} z_{t, n}(x, \boldsymbol{a})
$$

where

$$
z_{t, n}(x, \boldsymbol{a})=\mathbb{1}_{\eta_{t} \leq x \gamma_{t, n}(\boldsymbol{a})}-F\left(x \gamma_{t, n}(\boldsymbol{a})\right)-\left\{\mathbb{1}_{\eta_{t} \leq x}-F(x)\right\}
$$

We will establish a number of auxiliary lemmas.

Lemma 2. For any $u>0$ and sufficiently large $n$,

$$
P\left(\left|\frac{1}{\sqrt{n}} \sum_{t=1}^{n} z_{t, n}(x, \boldsymbol{a})\right|>u\right) \leq \frac{K}{n u^{4}}\left(x^{2}\|\boldsymbol{a}\|^{2}+1\right) .
$$

Proof. By the Markov inequality

$$
P\left(\left|\frac{1}{\sqrt{n}} \sum_{t=1}^{n} z_{t, n}(x, \boldsymbol{a})\right|>u\right) \leq \frac{1}{n^{2}} \frac{1}{u^{4}} E\left(\sum_{t=1}^{n} z_{t, n}(x, \boldsymbol{a})\right)^{4} .
$$

We note that, for fixed $n, x$ and $\boldsymbol{a},\left(z_{t, n}(x, \boldsymbol{a}), \mathcal{F}_{t}\right)_{1 \leq t \leq n}$ is a martingale difference sequence. Hence, by Rosenthal's inequality (see for instance Hall and Heyde (1980), Theorem 2.11)

$$
E\left(\sum_{t=1}^{n} z_{t, n}(x, \boldsymbol{a})\right)^{4} \leq K\left\{E\left(\sum_{t=1}^{n} E\left(z_{t, n}^{2}(x, \boldsymbol{a}) \mid \mathcal{F}_{t-1}\right)\right)^{2}+\sum_{t=1}^{n} E z_{t, n}^{4}(x, \boldsymbol{a})\right\}
$$

Because $\left|z_{t, n}(x, \boldsymbol{a})\right| \leq 2$, it suffices to show that

$$
E\left(\sum_{t=1}^{n} E\left(z_{t, n}^{2}(x, \boldsymbol{a}) \mid \mathcal{F}_{t-1}\right)\right)^{2} \leq n K x^{2}\|\boldsymbol{a}\|^{2} .
$$

Noting that the second-order conditional moment of $z_{t, n}(x, \boldsymbol{a})$ is the variance of a Bernoulli distribution, we have, using A11,

$$
\sum_{t=1}^{n} E\left[z_{t, n}^{2}(x, \boldsymbol{a}) \mid \mathcal{F}_{t-1}\right] \leq \sum_{t=1}^{n}\left|F\left(x \gamma_{t, n}(\boldsymbol{a})\right)-F(x)\right| \leq \frac{K|x|}{\sqrt{n}} \sum_{t=1}^{n}\left\|\frac{1}{\sigma_{t}\left(\boldsymbol{\theta}_{0}\right)} \frac{\partial \sigma_{t}\left(\boldsymbol{\theta}_{t}^{*}\right)}{\partial \boldsymbol{\theta}}\right\|\|\boldsymbol{a}\|,
$$


where $\boldsymbol{\theta}_{t}^{*}$ is between $\boldsymbol{\theta}_{0}$ and $\boldsymbol{\theta}_{0}+\boldsymbol{a} / \sqrt{n}$. It follows that

$$
E\left\{\sum_{t=1}^{n} E\left[z_{t, n}^{2}(x, \boldsymbol{a}) \mid \mathcal{F}_{t-1}\right]\right\}^{2} \leq \frac{K x^{2}}{n} \sum_{s, t=1}^{n} E\left\|\frac{1}{\sigma_{s}\left(\boldsymbol{\theta}_{0}\right)} \frac{\partial \sigma_{s}\left(\boldsymbol{\theta}_{s}^{*}\right)}{\partial \boldsymbol{\theta}}\right\|\left\|\frac{1}{\sigma_{t}\left(\boldsymbol{\theta}_{0}\right)} \frac{\partial \sigma_{t}\left(\boldsymbol{\theta}_{t}^{*}\right)}{\partial \boldsymbol{\theta}}\right\|\|\boldsymbol{a}\|^{2},
$$

and thus (13) holds.

Lemma 3. Let $\mathcal{K}$ a compact subset of $\mathbb{R}$. We have $\sup _{x \in \mathcal{K}}\left|n^{-1 / 2} \sum_{t=1}^{n} z_{t, n}(x, \boldsymbol{a})\right|=o_{P}(1)$.

Proof. Fix $\varepsilon>0$ and let $\mathcal{K} \subset\left[-\frac{N \varepsilon}{\sqrt{n}}, \frac{N \varepsilon}{\sqrt{n}}\right]$ with $N=O(\sqrt{n})$. Define $x_{j}=\frac{j \varepsilon}{\sqrt{n}}$ for $j=-N,-N+$ $1, \ldots, N-1, N$. It follows that, by Lemma 2 , for any $u>0$, there exists $K=K(u, \boldsymbol{a}, \varepsilon)$ such that

$$
P\left(\max _{-N \leq j \leq N}\left|\frac{1}{\sqrt{n}} \sum_{t=1}^{n} z_{t, n}\left(x_{j}, \boldsymbol{a}\right)\right|>u\right) \leq \frac{K}{\sqrt{n}} .
$$

Noting that

$$
\begin{aligned}
\sup _{x \in \mathcal{K}}\left|\frac{1}{\sqrt{n}} \sum_{t=1}^{n} z_{t, n}(x, \boldsymbol{a})\right| \leq & \max _{-N \leq j \leq N-1} \sup _{x \in\left[x_{j}, x_{j+1}\right]}\left|\frac{1}{\sqrt{n}} \sum_{t=1}^{n} z_{t, n}(x, \boldsymbol{a})\right| \\
\leq & \max _{-N \leq j \leq N-1}\left\{\sup _{x \in\left[x_{j}, x_{j+1}\right]}\left|\frac{1}{\sqrt{n}} \sum_{t=1}^{n} z_{t, n}(x, \boldsymbol{a})-\frac{1}{\sqrt{n}} \sum_{t=1}^{n} z_{t, n}\left(x_{j}, \boldsymbol{a}\right)\right|\right. \\
& \left.+\left|\frac{1}{\sqrt{n}} \sum_{t=1}^{n} z_{t, n}\left(x_{j}, \boldsymbol{a}\right)\right|\right\},
\end{aligned}
$$

it remains to show that

$$
\limsup _{n \rightarrow \infty} P\left\{\max _{-N \leq j \leq N-1} \sup _{x \in\left[x_{j}, x_{j+1}\right]}\left|\frac{1}{\sqrt{n}} \sum_{t=1}^{n} z_{t, n}(x, \boldsymbol{a})-\frac{1}{\sqrt{n}} \sum_{t=1}^{n} z_{t, n}\left(x_{j}, \boldsymbol{a}\right)\right|>u\right\}=0 .
$$

We have, for $j=0, \ldots, N-1$,

$$
\begin{aligned}
& \sup _{x \in\left[x_{j}, x_{j+1}\right]} \frac{1}{\sqrt{n}}\left|\sum_{t=1}^{n} \mathbb{1}_{\eta_{t} \leq x \gamma_{t, n}(\boldsymbol{a})}-F\left(x \gamma_{t, n}(\boldsymbol{a})\right)-\left\{\mathbb{1}_{\eta_{t} \leq x_{j} \gamma_{t, n}(\boldsymbol{a})}-F\left(x_{j} \gamma_{t, n}(\boldsymbol{a})\right)\right\}\right| \\
\leq & \sup _{x \in\left[x_{j}, x_{j+1}\right]} \frac{1}{\sqrt{n}} \sum_{t=1}^{n} \mathbb{1}_{\eta_{t} \leq x \gamma_{t, n}(\boldsymbol{a})}-\mathbb{1}_{\eta_{t} \leq x_{j} \gamma_{t, n}(\boldsymbol{a})} \\
& +\sup _{x \in\left[x_{j}, x_{j+1}\right]} \frac{1}{\sqrt{n}} \sum_{t=1}^{n} F\left(x \gamma_{t, n}(\boldsymbol{a})\right)-F\left(x_{j} \gamma_{t, n}(\boldsymbol{a})\right) \\
\leq & \frac{1}{\sqrt{n}} \sum_{t=1}^{n} \mathbb{1}_{\eta_{t} \leq x_{j+1} \gamma_{t, n}(\boldsymbol{a})}-\mathbb{1}_{\eta_{t} \leq x_{j} \gamma_{t, n}(\boldsymbol{a})}+\frac{1}{\sqrt{n}} \sum_{t=1}^{n} F\left(x_{j+1} \gamma_{t, n}(\boldsymbol{a})\right)-F\left(x_{j} \gamma_{t, n}(\boldsymbol{a})\right) \\
\leq & \left|\frac{1}{\sqrt{n}} \sum_{t=1}^{n}\left\{\mathbb{1}_{\eta_{t} \leq x_{j+1} \gamma_{t, n}(\boldsymbol{a})}-F\left(x_{j+1} \gamma_{t, n}(\boldsymbol{a})\right)\right\}-\left\{\mathbb{1}_{\eta_{t} \leq x_{j} \gamma_{t, n}(\boldsymbol{a})}-F\left(x_{j} \gamma_{t, n}(\boldsymbol{a})\right)\right\}\right| \\
& +2 W_{n}(j, \boldsymbol{a}),
\end{aligned}
$$


where $W_{n}(j, \boldsymbol{a})=n^{-1 / 2} \sum_{t=1}^{n}\left\{F\left(x_{j+1} \gamma_{t, n}(\boldsymbol{a})\right)-F\left(x_{j} \gamma_{t, n}(\boldsymbol{a})\right)\right\}$. Therefore,

$$
\begin{aligned}
& \sup _{x \in\left[x_{j}, x_{j+1}\right]} \frac{1}{\sqrt{n}}\left|\sum_{t=1}^{n} \mathbb{1}_{\eta_{t} \leq x \gamma_{t, n}(\boldsymbol{a})}-F\left(x \gamma_{t, n}(\boldsymbol{a})\right)-\left\{\mathbb{1}_{\eta_{t} \leq x_{j} \gamma_{t, n}(\boldsymbol{a})}-F\left(x_{j} \gamma_{t, n}(\boldsymbol{a})\right)\right\}\right| \\
\leq & \frac{1}{\sqrt{n}}\left|\sum_{t=1}^{n} z_{t, n}\left(x_{j+1}, \boldsymbol{a}\right)\right|+\frac{1}{\sqrt{n}}\left|\sum_{t=1}^{n} z_{t, n}\left(x_{j}, \boldsymbol{a}\right)\right|+V_{n}(j)+2 W_{n}(j, \boldsymbol{a}),
\end{aligned}
$$

where $V_{n}(j)=n^{-1 / 2}\left|\sum_{t=1}^{n}\left\{\mathbb{1}_{\eta_{t} \leq x_{j+1}}-F\left(x_{j+1}\right)\right\}-\left\{\mathbb{1}_{\eta_{t} \leq x_{j}}-F\left(x_{j}\right)\right\}\right|$. By Assumption A11 and the mean-value theorem, $F\left(x_{j+1}\right)-F\left(x_{j}\right) \leq M \varepsilon / \sqrt{n}$ where $M=\sup _{x \in \mathbb{R}} f(x)$. From (16) with $\boldsymbol{a}=\mathbf{0}$, it follows that

$$
\begin{aligned}
& \sup _{x \in\left[x_{j}, x_{j+1}\right]} \frac{1}{\sqrt{n}}\left|\sum_{t=1}^{n} \mathbb{1}_{\eta_{t} \leq x}-F(x)-\left\{\mathbb{1}_{\eta_{t} \leq x_{j}}-F\left(x_{j}\right)\right\}\right| \\
& \leq V_{n}(j)+2 \sqrt{n}\left\{F\left(x_{j+1}\right)-F\left(x_{j}\right)\right\} \leq V_{n}(j)+2 M \varepsilon .
\end{aligned}
$$

Therefore, for $j=0, \ldots, N-1$, from (16)-(17),

$$
\begin{aligned}
& \sup _{x \in\left[x_{j}, x_{j+1}\right]}\left|\frac{1}{\sqrt{n}} \sum_{t=1}^{n} z_{t, n}(x, \boldsymbol{a})-\frac{1}{\sqrt{n}} \sum_{t=1}^{n} z_{t, n}\left(x_{j}, \boldsymbol{a}\right)\right| \\
\leq & \frac{1}{\sqrt{n}}\left|\sum_{t=1}^{n} z_{t, n}\left(x_{j+1}, \boldsymbol{a}\right)\right|+\frac{1}{\sqrt{n}}\left|\sum_{t=1}^{n} z_{t, n}\left(x_{j}, \boldsymbol{a}\right)\right|+2 W_{n}(j, \boldsymbol{a})+2 V_{n}(j)+2 M \varepsilon,
\end{aligned}
$$

so

$$
\begin{aligned}
& \max _{0 \leq j \leq N-1} \sup _{x \in\left[x_{j}, x_{j+1}\right]}\left|\frac{1}{\sqrt{n}} \sum_{t=1}^{n} z_{t, n}(x, \boldsymbol{a})-\frac{1}{\sqrt{n}} \sum_{t=1}^{n} z_{t, n}\left(x_{j}, \boldsymbol{a}\right)\right| \\
\leq & \max _{0 \leq j \leq N} \frac{2}{\sqrt{n}}\left|\sum_{t=1}^{n} z_{t, n}\left(x_{j}, \boldsymbol{a}\right)\right|+2 \max _{0 \leq j \leq N-1} W_{n}(j, \boldsymbol{a})+2 \max _{0 \leq j \leq N-1} V_{n}(j) \\
& +2 M \varepsilon .
\end{aligned}
$$

By the properties of the modulus of continuity of the empirical process (see Shorack and Wellner (1986), p. 542), under Assumption A11 we have

$$
\max _{0 \leq j \leq N-1} V_{n}(j)=o_{P}(1) .
$$

Now, using again the mean-value theorem,

$$
\begin{aligned}
\max _{0 \leq j \leq N-1} W_{n}(j, \boldsymbol{a}) & \leq \max _{0 \leq j \leq N-1} \frac{1}{\sqrt{n}} \sum_{t=1}^{n}\left(x_{j+1}-x_{j}\right) \gamma_{t, n}(\boldsymbol{a}) M \\
& \leq \frac{M \varepsilon}{n} \sum_{t=1}^{n} \gamma_{t, n}(\boldsymbol{a}) \leq \frac{M \varepsilon}{n} \sum_{t=1}^{n}\left(1+\left\|\frac{1}{\sigma_{t}\left(\boldsymbol{\theta}_{0}\right)} \frac{\partial \sigma_{t}\left(\boldsymbol{\theta}_{t}^{*}\right)}{\partial \boldsymbol{\theta}}\right\| \frac{\|\boldsymbol{a}\|}{\sqrt{n}}\right),
\end{aligned}
$$

where $\boldsymbol{\theta}_{t}^{*}$ is between $\boldsymbol{\theta}_{0}$ and $\boldsymbol{\theta}_{0}+\boldsymbol{a} / \sqrt{n}$. Thus

$$
\max _{0 \leq j \leq N-1} W_{n}(j, \boldsymbol{a}) \leq \frac{M \varepsilon}{n} \sum_{t=1}^{n}\left(1+\sup _{\boldsymbol{\theta} \in V\left(\boldsymbol{\theta}_{0}\right)}\left(\frac{\sigma_{t}(\boldsymbol{\theta})}{\sigma_{t}\left(\boldsymbol{\theta}_{0}\right)}\right) \sup _{\boldsymbol{\theta} \in V\left(\boldsymbol{\theta}_{0}\right)}\left\|\frac{1}{\sigma_{t}(\boldsymbol{\theta})} \frac{\partial \sigma_{t}(\boldsymbol{\theta})}{\partial \boldsymbol{\theta}}\right\| \frac{\|\boldsymbol{a}\|}{\sqrt{n}}\right)
$$




$$
=\varepsilon \times O_{P}(1) \text {, }
$$

by Assumption A9 and the ergodic theorem. Thus (14), (19) and (20) show that the right-hand side of $(18)$ is an $o_{P}(1)$. It is clear that the same bound can be obtained when $\max _{0 \leq j \leq N-1}$ is replaced by $\max _{-N \leq j \leq-1}$. Thus (15) is established.

Lemma 4. Let $\mathcal{K}$ be a compact subset of $\mathbb{R}$. For any $A>0$ and $\boldsymbol{A}=[-A, A]^{d}$,

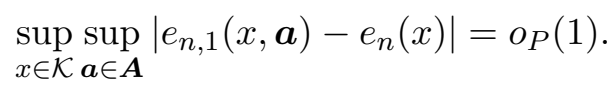

Proof. In view of (12) will show that

$$
\sup _{\boldsymbol{a} \in \boldsymbol{A}} X_{n}(\boldsymbol{a})=o_{P}(1), \quad \text { where } \quad X_{n}(\boldsymbol{a})=\sup _{x \in \mathcal{K}}\left|\frac{1}{\sqrt{n}} \sum_{t=1}^{n} z_{t, n}(x, \boldsymbol{a})\right| .
$$

Let $\varepsilon>0$ such that $N:=2 A / \varepsilon$ is an integer and define $a(k)=-A+k \varepsilon$, for $1 \leq k \leq N$. For any $1 \leq$ $k_{1}, k_{2}, \ldots k_{d} \leq N$ let $\boldsymbol{k}=\left(k_{1}, \ldots, k_{d}\right)$ and consider the grid of $N^{d}$ points $\boldsymbol{a}(\boldsymbol{k})=\left(a\left(k_{1}\right), \ldots, a\left(k_{d}\right)\right)$. Let also $\boldsymbol{A}(\boldsymbol{k})=\left\{\left(a_{1}, \ldots, a_{d}\right) \in \boldsymbol{A} \mid a\left(k_{i}\right)-\varepsilon \leq a_{i} \leq a\left(k_{i}\right)\right\}$ and $\boldsymbol{a}^{*}(\boldsymbol{k})=\left(a\left(k_{1}\right)-\varepsilon, \ldots, a\left(k_{d}\right)-\varepsilon\right)$. We have, for $j=1, \ldots, d$ and $a_{j} \leq a\left(k_{j}\right)$

$$
\begin{aligned}
& F\left(x \gamma_{t, n}\left(a_{1}, \ldots, a_{j-1}, a\left(k_{j}\right), a_{j+1}, \ldots, a_{d}\right)\right)-F\left(x \gamma_{t, n}\left(a_{1}, \ldots, a_{j-1}, a_{j}, a_{j+1}, \ldots, a_{d}\right)\right) \\
= & f\left\{x \gamma_{t, n}\left(\boldsymbol{a}_{t, j}^{*}\right)\right\} \frac{x}{\sqrt{n}}\left(a\left(k_{j}\right)-a_{j}\right) \frac{1}{\sigma_{t}\left(\boldsymbol{\theta}_{0}\right)} \frac{\partial \sigma_{t}\left(\boldsymbol{\theta}_{0}+\frac{\boldsymbol{a}_{t, j}^{*}}{\sqrt{n}}\right)}{\partial \boldsymbol{\theta}^{\prime}} \boldsymbol{e}_{j},
\end{aligned}
$$

where $\boldsymbol{e}_{j}$ is the $j$-th element of the canonical basis of $\mathbb{R}^{d}$, and $\boldsymbol{a}_{t, j}^{*}$ is a point between the arguments of $\gamma_{t, n}$ above. By A11 and $E\left|\eta_{t}\right|<\infty$, we have $\sup _{x}|x| f(x)<\infty$. The latter difference is thus bounded, uniformly in $x \in \mathbb{R}$ and $a_{j} \in\left[a\left(k_{j}\right)-\varepsilon, a\left(k_{j}\right)\right]$, by

$$
K \frac{\varepsilon}{\sqrt{n}} \frac{1}{\sigma_{t}\left(\boldsymbol{\theta}_{0}+\frac{\boldsymbol{a}_{t, j}^{*}}{\sqrt{n}}\right)} \frac{\partial \sigma_{t}\left(\boldsymbol{\theta}_{0}+\frac{\boldsymbol{a}_{t, j}^{*}}{\sqrt{n}}\right)}{\partial \boldsymbol{\theta}^{\prime}} \boldsymbol{e}_{j} .
$$

Therefore, for $n$ large enough,

$$
\sup _{\boldsymbol{a} \in \boldsymbol{A}(\boldsymbol{k})} \sup _{x \in \mathbb{R}} \sum_{t=1}^{n}\left|F\left(x \gamma_{t, n}(\boldsymbol{a})\right)-F\left(x \gamma_{t, n}(\boldsymbol{a}(\boldsymbol{k}))\right)\right| \leq K \frac{\varepsilon}{\sqrt{n}} \sum_{t=1}^{n} \sup _{\boldsymbol{\theta} \in V\left(\boldsymbol{\theta}_{0}\right)}\left\|\frac{1}{\sigma_{t}(\boldsymbol{\theta})} \frac{\partial \sigma_{t}(\boldsymbol{\theta})}{\partial \boldsymbol{\theta}}\right\|,
$$

and thus, because $\gamma_{t, n}(\cdot)$ is an increasing function of its arguments by $\mathbf{A 1 0}$,

$$
\begin{aligned}
& \sup _{\boldsymbol{a} \in \boldsymbol{A}(\boldsymbol{k})} \sup _{x \in \mathcal{K}}\left|\sum_{t=1}^{n} z_{t, n}(x, \boldsymbol{a})-z_{t, n}(x, \boldsymbol{a}(\boldsymbol{k}))\right| \\
\leq & K \frac{\varepsilon}{\sqrt{n}} \sum_{t=1}^{n} \sup _{\boldsymbol{\theta} \in V\left(\boldsymbol{\theta}_{0}\right)}\left\|\frac{1}{\sigma_{t}} \frac{\partial \sigma_{t}(\boldsymbol{\theta})}{\partial \boldsymbol{\theta}}\right\|+\sup _{x \in \mathcal{K}}\left|\sum_{t=1}^{n} \mathbb{1}_{\eta_{t} \leq x \gamma_{t, n}(\boldsymbol{a}(\boldsymbol{k}))}-\mathbb{1}_{\eta_{t} \leq x \gamma_{t, n}\left(\boldsymbol{a}^{*}(\boldsymbol{k})\right)}\right| \\
\leq & 2 K \frac{\varepsilon}{\sqrt{n}} \sum_{t=1}^{n} \sup _{\boldsymbol{\theta} \in V\left(\boldsymbol{\theta}_{0}\right)}\left\|\frac{1}{\sigma_{t}} \frac{\partial \sigma_{t}(\boldsymbol{\theta})}{\partial \boldsymbol{\theta}}\right\|+\sup _{x \in \mathcal{K}}\left|\sum_{t=1}^{n} z_{t, n}(x, \boldsymbol{a}(\boldsymbol{k}))\right|+\sup _{x \in \mathcal{K}}\left|\sum_{t=1}^{n} z_{t, n}\left(x, \boldsymbol{a}^{*}(\boldsymbol{k})\right)\right| .
\end{aligned}
$$


Note that

$$
\begin{aligned}
& \sup _{\boldsymbol{a} \in \boldsymbol{A}} X_{n}(\boldsymbol{a}) \\
\leq & \max _{\boldsymbol{k} \in\{1, \ldots, N\}^{d}} \sup _{\boldsymbol{a} \in \boldsymbol{A}(\boldsymbol{k})} \sup _{x \in \mathcal{K}}\left|\frac{1}{\sqrt{n}} \sum_{t=1}^{n}\left[z_{t, n}(x, \boldsymbol{a})-z_{t, n}(x, \boldsymbol{a}(\boldsymbol{k}))\right]\right|+\max _{\boldsymbol{k} \in\{1, \ldots, N\}^{d}} X_{n}(\boldsymbol{a}(\boldsymbol{k})) \\
\leq & \frac{2 K \varepsilon}{n} \sum_{t=1}^{n} \sup _{\boldsymbol{\theta} \in V\left(\boldsymbol{\theta}_{0}\right)}\left\|\frac{1}{\sigma_{t}} \frac{\partial \sigma_{t}(\boldsymbol{\theta})}{\partial \boldsymbol{\theta}}\right\|+2 \max _{\boldsymbol{k} \in\{1, \ldots, N\}^{d}} X_{n}(\boldsymbol{a}(\boldsymbol{k}))+\max _{\boldsymbol{k} \in\{1, \ldots, N\}^{d}} X_{n}\left(\boldsymbol{a}^{*}(\boldsymbol{k})\right) .
\end{aligned}
$$

By the ergodic theorem and $\mathbf{A 9}$, the first term in the r.h.s. is almost surely less than a constant times $\varepsilon$ when $n$ is large. The two other terms tend to zero in probability because $X_{n}(\boldsymbol{a})=o_{P}(1)$ by Lemma 3 and the maximas are over a finite number of points. Therefore (21) is established.

Lemma 5. Let $\mathcal{K}$ be a compact subset of $\mathbb{R}$. Then $\sup _{x \in \mathcal{K}}\left|\widehat{e}_{n, 2}(x)-h_{n}(x)\right| \rightarrow 0$ a.s.

Proof. A Taylor expansion yields, for $x_{t}^{*}=x \sigma_{t}\left(\boldsymbol{\theta}_{t}^{*}\right) / \sigma_{t}\left(\boldsymbol{\theta}_{0}\right)$ with $\boldsymbol{\theta}_{t}^{*}$ between $\widehat{\boldsymbol{\theta}}_{n}$ and $\boldsymbol{\theta}_{0}$,

$$
\begin{aligned}
& \left|\widehat{e}_{n, 2}(x)-h_{n}(x)\right| \\
= & \left|\frac{1}{\sqrt{n}} \sum_{t=1}^{n}\left\{F\left(x \chi_{t, n}\right)-F(x)-x f(x) \frac{1}{\sigma_{t}} \frac{\partial \sigma_{t}\left(\boldsymbol{\theta}_{0}\right)}{\partial \boldsymbol{\theta}^{\prime}}\left(\widehat{\boldsymbol{\theta}}_{n}-\boldsymbol{\theta}_{0}\right)\right\}\right| \\
\leq & |x| \frac{1}{n} \sum_{t=1}^{n}\left|f\left(x_{t}^{*}\right) \frac{1}{\sigma_{t}} \frac{\partial \sigma_{t}\left(\boldsymbol{\theta}_{t}^{*}\right)}{\partial \boldsymbol{\theta}}-f(x) \frac{1}{\sigma_{t}} \frac{\partial \sigma_{t}\left(\boldsymbol{\theta}_{0}\right)}{\partial \boldsymbol{\theta}}\right|\left\|\sqrt{n}\left(\widehat{\boldsymbol{\theta}}_{n}-\boldsymbol{\theta}_{0}\right)\right\| \\
\leq & |x|\left(\frac{1}{n} \sum_{t=1}^{n}\left|f\left(x_{t}^{*}\right)-f(x)\right|^{2}\right)^{1 / 2}\left(\frac{1}{n} \sum_{t=1}^{n}\left|\frac{1}{\sigma_{t}} \frac{\partial \sigma_{t}\left(\boldsymbol{\theta}_{t}^{*}\right)}{\partial \boldsymbol{\theta}}\right|\right)^{1 / 2}\left\|\sqrt{n}\left(\widehat{\boldsymbol{\theta}}_{n}-\boldsymbol{\theta}_{0}\right)\right\| \\
& +|x| f(x) \frac{1}{n} \sum_{t=1}^{n}\left|\frac{1}{\sigma_{t}} \frac{\partial \sigma_{t}\left(\boldsymbol{\theta}_{t}^{*}\right)}{\partial \boldsymbol{\theta}}-\frac{1}{\sigma_{t}} \frac{\partial \sigma_{t}\left(\boldsymbol{\theta}_{0}\right)}{\partial \boldsymbol{\theta}}\right|\left\|\sqrt{n}\left(\widehat{\boldsymbol{\theta}}_{n}-\boldsymbol{\theta}_{0}\right)\right\|
\end{aligned}
$$

by Cauchy-Schwarz inequality. We have, for any $\Delta>0$ and $n$ large enough

$$
\frac{1}{n} \sum_{t=1}^{n} \sup _{x \in \mathcal{K}}\left|f\left(x_{t}^{*}\right)-f(x)\right|^{2} \leq \frac{1}{n} \sum_{t=1}^{n} \sup _{\boldsymbol{\delta}:\|\boldsymbol{\delta}\| \leq \Delta} \sup _{x \in \mathcal{K}}\left|f\left(x \frac{\sigma_{t}\left(\boldsymbol{\theta}_{0}+\boldsymbol{\delta}\right)}{\sigma_{t}\left(\boldsymbol{\theta}_{0}\right)}\right)-f(x)\right|^{2}+\mathbb{1}_{\left\|\widehat{\boldsymbol{\theta}}_{n}-\boldsymbol{\theta}_{0}\right\|>\Delta} .
$$

The first term in the r.h.s. converges a.s. to

$$
E \sup _{\boldsymbol{\delta}:\|\boldsymbol{\delta}\| \leq \Delta} \sup _{x \in \mathcal{K}}\left|f\left(x \frac{\sigma_{t}\left(\boldsymbol{\theta}_{0}+\boldsymbol{\delta}\right)}{\sigma_{t}\left(\boldsymbol{\theta}_{0}\right)}\right)-f(x)\right|^{2}
$$

which can be made arbitrarily small for $\Delta$ small enough by the dominated convergence theorem. The last term in the r.h.s. is a.s. equal to zero for $n$ large enough. It follows that the first term is the r.h.s. of the inequality (22) tends to zero almost surely uniformly in $x \in \mathcal{K}$. The second term is handled similarly.

Lemma 6. For any $s>0$, we have $n^{1 / 2-s}\left\|\widehat{\boldsymbol{\theta}}_{n}-\boldsymbol{\theta}_{0}\right\|=o(1)$ a.s. 
Proof. This is a direct consequence of the Bahadur expansion

$$
\sqrt{n}\left(\widehat{\boldsymbol{\theta}}_{n}-\boldsymbol{\theta}_{0}\right)=\frac{-\boldsymbol{J}^{-1}}{2 \sqrt{n}} \sum_{t=1}^{n}\left(1-\eta_{t}^{2}\right) \boldsymbol{D}_{t}+o_{P}(1)
$$

and Lemma 1 with $X_{t}$ being any component of $\left(1-\eta_{t}^{2}\right) \boldsymbol{D}_{t}$ (noting that $E X_{t}^{2}<\infty$ under $\mathbf{A 9}$ ).

Lemma 7. We have $\sup _{x \in \mathbb{R}}\left|\widehat{e}_{n, 3}(x)\right|=o_{P}(1)$.

Proof. In view of A2-A3, we have

$$
\left|\eta_{t}\left(\widehat{\boldsymbol{\theta}}_{n}\right)-\tilde{\eta}_{t}\left(\widehat{\boldsymbol{\theta}}_{n}\right)\right|=\frac{\left|\tilde{\sigma}_{t}\left(\widehat{\boldsymbol{\theta}}_{n}\right)-\sigma_{t}\left(\widehat{\boldsymbol{\theta}}_{n}\right)\right|}{\tilde{\sigma}_{t}\left(\widehat{\boldsymbol{\theta}}_{n}\right) \sigma_{t}\left(\widehat{\boldsymbol{\theta}}_{n}\right)} \sigma_{t}\left|\eta_{t}\right| \leq K \rho^{t} \sigma_{t}\left|\eta_{t}\right|
$$

It follows that

$$
\begin{aligned}
\left|\mathbb{1}_{\eta_{t} \leq x \tilde{\chi}_{t, n}}-\mathbb{1}_{\eta_{t} \leq x \chi_{t, n}}\right| & =\left|\mathbb{1}_{\tilde{\eta}_{t}\left(\widehat{\boldsymbol{\theta}}_{n}\right) \leq x}-\mathbb{1}_{\eta_{t}\left(\widehat{\boldsymbol{\theta}}_{n}\right) \leq x}\right| \leq \mathbb{1}_{x-K \rho^{t} \sigma_{t}\left|\eta_{t}\right| \leq \eta_{t}\left(\widehat{\boldsymbol{\theta}}_{n}\right) \leq x+K \rho^{t} \sigma_{t}\left|\eta_{t}\right|} \mid \\
& \leq \mathbb{1}_{x-K \rho^{t / 3} \leq \eta_{t}\left(\widehat{\boldsymbol{\theta}}_{n}\right) \leq x+K \rho^{t / 3}}+\mathbb{1}_{\left|\eta_{t}\right| \geq 1 / \rho^{t / 3}}+\mathbb{1}_{\sigma_{t} \geq 1 / \rho^{t / 3}} .
\end{aligned}
$$

Now, by Markov's inequality and A1

$$
E \frac{1}{\sqrt{n}} \sum_{t=1}^{n} \mathbb{1}_{\sigma_{t} \geq 1 / \rho^{t / 3}}=\frac{1}{\sqrt{n}} \sum_{t=1}^{n} P\left(\rho^{t / 3} \sigma_{t}>1\right)=O\left(n^{-1 / 2}\right) .
$$

Similarly it can be shown that $n^{-1 / 2} \sum_{t=1}^{n} \mathbb{1}_{\left|\eta_{t}\right| \geq 1 / \rho^{t / 3}}=O_{P}\left(n^{-1 / 2}\right)$. It remains to show that

$$
\sup _{x \in \mathbb{R}} \frac{1}{\sqrt{n}} \sum_{t=1}^{n} d_{t, n}(x)=o_{P}(1), \quad d_{t, n}(x)=\mathbb{1}_{\chi_{t, n} x-K \chi_{t, n} \rho^{t / 3} \leq \eta_{t} \leq \chi_{t, n} x+K \chi_{t, n} \rho^{t / 3} .} .
$$

Let $\left(a_{n}\right)$ and $\left(b_{n}\right)$ be two sequences of real numbers, and let $s>0$. Consider the events $A_{n}=$ $\left\{\min _{1 \leq t<t^{\prime} \leq n}\left|\eta_{t}-\eta_{t^{\prime}}\right| \geq a_{n}\right\}$. We have $\sup _{x \in \mathbb{R}} n^{-1 / 2} \sum_{t=1}^{n} d_{t, n}(x) \leq \sum_{i=1}^{5} c_{i, n}$, with

$$
\begin{aligned}
c_{1, n} & =\sup _{x \in \mathbb{R}} \frac{1}{\sqrt{n}} \sum_{t=1}^{\left\lceil n^{1 / 4}\right\rceil-1} d_{t, n}(x) \leq \frac{1}{n^{1 / 4}}, \\
c_{2, n} & =\sup _{x \in \mathbb{R}} \frac{1}{\sqrt{n}} \sum_{t=\left\lceil n^{1 / 4}\right\rceil}^{n} \mathbb{1}_{\chi_{t, n} x-K b_{n} \rho_{n} \leq \eta_{t} \leq \chi_{t, n} x+K b_{n} \rho_{n}} \mathbb{1}_{A_{n}}, \quad \rho_{n}=\rho^{\frac{n^{1 / 4}}{3}}, \\
c_{3, n} & =\frac{1}{\sqrt{n}} \sum_{t=1}^{n} \mathbb{1}_{\chi_{t, n} \geq b_{n}} \mathbb{1}_{\sqrt{n}\left\|\widehat{\boldsymbol{\theta}}_{n}-\boldsymbol{\theta}_{0}\right\| \leq n^{s}}, \quad c_{4, n}=\sqrt{n} \mathbb{1}_{\sqrt{n}\left\|\widehat{\boldsymbol{\theta}}_{n}-\boldsymbol{\theta}_{0}\right\| \geq n^{s}}, \quad c_{5, n}=\sqrt{n} \mathbb{1}_{A_{n}^{c}} .
\end{aligned}
$$

Note that $c_{2, n}=1 / \sqrt{n}$ when $a_{n} \geq 2 K b_{n} \rho_{n}$. Now, for some $\boldsymbol{\theta}_{t}$ between $\widehat{\boldsymbol{\theta}}_{n}$ and $\boldsymbol{\theta}_{0}$, we have

$$
\chi_{t, n}=1+\frac{1}{\sigma_{t}\left(\boldsymbol{\theta}_{0}\right)} \frac{\partial \sigma_{t}\left(\boldsymbol{\theta}_{t}\right)}{\partial \boldsymbol{\theta}}\left(\widehat{\boldsymbol{\theta}}_{n}-\boldsymbol{\theta}_{0}\right) .
$$

Using $\mathbf{A 9}$ and assuming $b_{n}>1$, it follows that

$$
E c_{3, n}=\frac{1}{\sqrt{n}} \sum_{t=1}^{n} P\left(\chi_{t, n} \geq b_{n}, \sqrt{n}\left\|\widehat{\boldsymbol{\theta}}_{n}-\boldsymbol{\theta}_{0}\right\| \leq n^{s}\right)
$$




$$
\leq \frac{1}{\sqrt{n}} \sum_{t=1}^{n} P\left(\sup _{\boldsymbol{\theta} \in V\left(\boldsymbol{\theta}_{0}\right)}\left\|\frac{1}{\sigma_{t}\left(\boldsymbol{\theta}_{0}\right)} \frac{\partial \sigma_{t}(\boldsymbol{\theta})}{\partial \boldsymbol{\theta}}\right\| n^{s-1 / 2} \geq b_{n}-1\right) \leq K \frac{n^{2 s}}{\sqrt{n}\left(b_{n}-1\right)^{2}}
$$

By Lemma 6, we have $c_{4, n}=0$ a.s. for $n$ large enough. Since $f$ is bounded under $\mathbf{A 1 1}$, we have

$$
P\left(\left|\eta_{1}-\eta_{2}\right|<a_{n}\right)=\int\left(\int_{x-a_{n}}^{x+a_{n}} f(y) d y\right) f(x) d x \leq K a_{n} .
$$

Therefore $E c_{5, n}=\sqrt{n} P\left(A_{n}^{c}\right) \leq \sqrt{n} K\left(\begin{array}{c}n \\ 2\end{array}\right) a_{n} \leq K n^{2+1 / 2} a_{n}$. By taking, for instance, $a_{n}=n^{-3}$, $b_{n}=\sqrt{n}$ and $s<1 / 2$, it can be checked that $c_{i, n}=o_{P}(1)$ for $i=1, \ldots, 5$. We conclude that

$$
\left|\widehat{e}_{n, 3}(x)\right| \leq \frac{1}{\sqrt{n}} \sum_{t=1}^{n}\left|\mathbb{1}_{\eta_{t} \leq x \tilde{\chi}_{t, n}}-\mathbb{1}_{\eta_{t} \leq x \chi_{t, n}}\right|=o_{P}(1) .
$$

Lemma 8. Let $\mathcal{K}$ be a compact subset of $\mathbb{R}$. We have $\sup _{x \in \mathcal{K}}\left|\widehat{e}_{n}(x)-e_{n}(x)-h_{n}(x)\right|=o_{P}(1)$.

Proof. We have

$$
\left|\widehat{e}_{n}(x)-e_{n}(x)-h_{n}(x)\right| \leq\left|\widehat{e}_{n, 1}(x)-e_{n}(x)\right|+\left|\widehat{e}_{n, 2}(x)-h_{n}(x)\right|+\left|\widehat{e}_{n, 3}(x)\right|
$$

Note that $\widehat{e}_{n, 1}(x)=e_{n, 1}\left(x, \sqrt{n}\left(\widehat{\boldsymbol{\theta}}_{n}-\boldsymbol{\theta}_{0}\right)\right)$. Thus, any $u, A>0$ and $\boldsymbol{A}=[-A, A]^{d}$,

$$
\begin{aligned}
P\left(\sup _{x \in \mathcal{K}}\left|\widehat{e}_{n, 1}(x)-e_{n}(x)\right|>u\right) \leq & P\left(\sup _{x \in \mathcal{K}} \sup _{\boldsymbol{a} \in \boldsymbol{A}}\left|e_{n, 1}(x, \boldsymbol{a})-e_{n}(x)\right|>u\right) \\
& +P\left(\sqrt{n}\left(\widehat{\boldsymbol{\theta}}_{n}-\boldsymbol{\theta}_{0}\right) \notin \boldsymbol{A}\right) .
\end{aligned}
$$

By Lemma 4, the first term in the r.h.s. tends to zero, while the second term can be made arbitrarily small by letting $A \rightarrow \infty$. Thus $\sup _{x \in \mathcal{K}}\left|\widehat{e}_{n, 1}(x)-e_{n}(x)\right|=o_{P}(1)$. The conclusion follows using Lemmas 5 and 7 .

The stochastic equicontinuity property stated in the following lemma follows from Billingsley (1968) (see Appendix).

Lemma 9. If $x$ is a real number and $\left(x_{n}\right)$ is a sequence of real random variables such that $x_{n} \rightarrow x$ in probability, and if $F$ is continuous, then $e_{n}\left(x_{n}\right)-e_{n}(x)=o_{P}(1)$.

Now we complete the proof of Theorem 2 establishing the asymptotic distribution of $\widehat{e}_{n}\left(x_{n}\right)$. By A11, $h_{n}\left(x_{n}\right)-h_{n}(x)=o_{P}(1)$. By Lemmas 8-9 and (23), it then follows that

$$
\begin{aligned}
\widehat{e}_{n}\left(x_{n}\right) & =e_{n}\left(x_{n}\right)+h_{n}\left(x_{n}\right)+o_{P}(1) \\
& =\frac{1}{\sqrt{n}} \sum_{t=1}^{n}\left\{\mathbb{1}_{\eta_{t}<x}-F(x)\right\}-\frac{x f(x)}{2 \sqrt{n}} \boldsymbol{\Omega}^{\prime} \boldsymbol{J}^{-1} \sum_{t=1}^{n}\left(1-\eta_{t}^{2}\right) \boldsymbol{D}_{t}+o_{P}(1) .
\end{aligned}
$$

We conclude by using $\boldsymbol{\Omega}^{\prime} \boldsymbol{J}^{-1} \boldsymbol{\Omega}=1$ under A12 (see Remark 3 in Francq and Zakoian, 2013a). 


\subsection{Proof of Theorem 4}

By comparison with the proof of Theorem 2, the major difficulty comes from the non-monotonicity of the conditional mean and variance with respect to the parameters (A10 can no longer be assumed). To solve this difficulty we will introduce a partition of the past, so that the mean and variance are monotonous conditional on this partition.

We recall some notations and introduce new ones. Let

$$
\begin{aligned}
\widehat{e}_{n}(x) & =\sqrt{n}\left\{\widehat{F}_{n}(x)-F(x)\right\}, \quad e_{n}(x)=\sqrt{n}\left\{F_{n}(x)-F(x)\right\}, \quad \tilde{\chi}_{t, n}=\frac{\tilde{\sigma}_{t}\left(\widehat{\boldsymbol{\theta}}_{n}\right)}{\sigma_{t}\left(\boldsymbol{\theta}_{0}\right)}, \\
\chi_{t, n} & =\frac{\sigma_{t}\left(\widehat{\boldsymbol{\theta}}_{n}\right)}{\sigma_{t}\left(\boldsymbol{\theta}_{0}\right)}, \quad \tilde{\lambda}_{t, n}=\frac{\tilde{m}_{t}\left(\widehat{\boldsymbol{\theta}}_{n}\right)-m_{t}\left(\boldsymbol{\theta}_{0}\right)}{\sigma_{t}\left(\boldsymbol{\theta}_{0}\right)}, \quad \lambda_{t, n}=\frac{m_{t}\left(\widehat{\boldsymbol{\theta}}_{n}\right)-m_{t}\left(\boldsymbol{\theta}_{0}\right)}{\sigma_{t}\left(\boldsymbol{\theta}_{0}\right)}, \\
\chi_{t, n}^{*} & =1+\frac{1}{\sigma_{t}\left(\boldsymbol{\theta}_{0}\right)} \frac{\partial \sigma_{t}\left(\boldsymbol{\theta}_{0}\right)}{\partial \boldsymbol{\theta}^{\prime}}\left(\widehat{\boldsymbol{\theta}}_{n}-\boldsymbol{\theta}_{0}\right), \quad \lambda_{t, n}^{*}=\frac{1}{\sigma_{t}\left(\boldsymbol{\theta}_{0}\right)} \frac{\partial m_{t}\left(\boldsymbol{\theta}_{0}\right)}{\partial \boldsymbol{\theta}^{\prime}}\left(\widehat{\boldsymbol{\theta}}_{n}-\boldsymbol{\theta}_{0}\right), \\
H_{n}(x) & =f(x)\left(x\left\{\frac{1}{n} \sum_{t=1}^{n} \frac{1}{\sigma_{t}} \frac{\partial \sigma_{t}\left(\boldsymbol{\theta}_{0}\right)}{\partial \boldsymbol{\theta}^{\prime}}\right\}+\left\{\frac{1}{n} \sum_{t=1}^{n} \frac{1}{\sigma_{t}} \frac{\partial m_{t}\left(\boldsymbol{\theta}_{0}\right)}{\partial \boldsymbol{\theta}^{\prime}}\right\}\right) \sqrt{n}\left(\widehat{\boldsymbol{\theta}}_{n}-\boldsymbol{\theta}_{0}\right) .
\end{aligned}
$$

We have

$$
\begin{aligned}
\widehat{e}_{n}(x)= & \underbrace{\frac{1}{\sqrt{n}} \sum_{t=1}^{n} \mathbb{1}_{\eta_{t} \leq x \chi_{t, n}^{*}+\lambda_{t, n}^{*}}-F\left(x \chi_{t, n}^{*}+\lambda_{t, n}^{*}\right)}_{\widehat{e}_{n, 4}(x)}+\underbrace{\frac{1}{\sqrt{n}} \sum_{t=1}^{n} F\left(x \chi_{t, n}^{*}+\lambda_{t, n}^{*}\right)-F(x)}_{\widehat{e}_{n, 5}(x)} \\
& +\underbrace{\frac{1}{\sqrt{n} \sum_{t=1}^{n} \mathbb{1}_{\eta_{t} \leq x \tilde{\chi}_{t, n}+\tilde{\lambda}_{t, n}}-\mathbb{1}_{\eta_{t} \leq x \chi_{t, n}^{*}+\lambda_{t, n}^{*}}}}_{\widehat{e}_{n, 6}(x)} .
\end{aligned}
$$

Let, for $\boldsymbol{a}$ a vector of the same size as $\boldsymbol{\theta}$, sufficiently small so that $\boldsymbol{\theta}_{0}+\boldsymbol{a} / \sqrt{n} \in \boldsymbol{\Theta}$,

$$
\begin{array}{r}
\lambda_{t, n}(\boldsymbol{a})=\frac{m_{t}\left(\boldsymbol{\theta}_{0}+\frac{\boldsymbol{a}}{\sqrt{n}}\right)-m_{t}\left(\boldsymbol{\theta}_{0}\right)}{\sigma_{t}\left(\boldsymbol{\theta}_{0}\right)}, \quad \gamma_{t, n}(\boldsymbol{a})=\frac{\sigma_{t}\left(\boldsymbol{\theta}_{0}+\frac{\boldsymbol{a}}{\sqrt{n}}\right)}{\sigma_{t}\left(\boldsymbol{\theta}_{0}\right)}, \\
e_{n, 4}(x, \boldsymbol{a})=\frac{1}{\sqrt{n}} \sum_{t=1}^{n}\left\{\mathbb{1}_{\eta_{t} \leq x \gamma_{t, n}^{*}(\boldsymbol{a})+\lambda_{t, n}^{*}(\boldsymbol{a})}-F\left(x \gamma_{t, n}^{*}(\boldsymbol{a})+\lambda_{t, n}^{*}(\boldsymbol{a})\right)\right\}, \\
\lambda_{t, n}^{*}(\boldsymbol{a})=\frac{1}{\sigma_{t}\left(\boldsymbol{\theta}_{0}\right)} \frac{\partial m_{t}\left(\boldsymbol{\theta}_{0}\right)}{\partial \boldsymbol{\theta}^{\prime}} \frac{\boldsymbol{a}}{\sqrt{n}}, \quad \gamma_{t, n}^{*}(\boldsymbol{a})=1+\frac{1}{\sigma_{t}\left(\boldsymbol{\theta}_{0}\right)} \frac{\partial \sigma_{t}\left(\boldsymbol{\theta}_{0}\right)}{\partial \boldsymbol{\theta}^{\prime}} \frac{\boldsymbol{a}}{\sqrt{n}} .
\end{array}
$$

For $\boldsymbol{a}, \boldsymbol{b} \in \mathbb{R}^{d}$ let

$$
z_{t, n}^{*}(x, \boldsymbol{a}, \boldsymbol{b})=\mathbb{1}_{\eta_{t} \leq x \gamma_{t, n}^{*}(\boldsymbol{a})+\lambda_{t, n}^{*}(\boldsymbol{b})}-F\left(x \gamma_{t, n}^{*}(\boldsymbol{a})+\lambda_{t, n}^{*}(\boldsymbol{b})\right)-\left\{\mathbb{1}_{\eta_{t} \leq x}-F(x)\right\} .
$$

Write $y_{t-1}=\left(y_{t-1}, y_{t-2}, \ldots\right)$. Let $\mathcal{S}=\left\{s_{1}, \ldots, s_{2^{2 d}}\right\}$ the elements of $\{-1,1\}^{2 d}$ taken in the lexicographical order (thus $\boldsymbol{s}_{1}=(-1, \ldots,-1)$ and $\boldsymbol{s}_{2^{2 d}}=(1, \ldots, 1)$ ). For any vector $\boldsymbol{Z} \in \mathbb{R}^{2 d}$, let $\boldsymbol{s}[\boldsymbol{Z}]=\left(s_{1}, \ldots, s_{2 d}\right)$ denote the element of $\mathcal{S}$ such that $s_{i}=\operatorname{sgn}\left(Z_{i}\right), i=1, \ldots, 2 d$. Define $\left\{\mathcal{R}_{i}\right\}_{i \in I}$ a partition of $\mathbb{R}^{\infty}$, with $I=\left\{1, \ldots, 2^{2 d}\right\}$ such that, for $i \in I$,

$$
y_{\underline{t-1}} \in \mathcal{R}_{i} \quad \Leftrightarrow \quad \boldsymbol{s}\left[\left(\frac{\partial m_{t}}{\partial \boldsymbol{\theta}^{\prime}}\left(\boldsymbol{\theta}_{0}\right), \frac{\partial \sigma_{t}}{\partial \boldsymbol{\theta}^{\prime}}\left(\boldsymbol{\theta}_{0}\right)\right)^{\prime}\right]=\boldsymbol{s}_{i}:=\left(\boldsymbol{s}_{i}^{(1)}, \boldsymbol{s}_{i}^{(2)}\right)
$$


where $\boldsymbol{s}_{i}^{(j)} \in\{-1,1\}^{d}, j=1,2$.

Lemma 10. We have, for any $u>0$ and sufficiently large $n$, for any sequences $\left(\boldsymbol{a}_{i}\right)_{i \in I}$ and $\left(\boldsymbol{b}_{i}\right)_{i \in I}$

$$
P\left(\left|\sum_{i \in I} \frac{1}{\sqrt{n}} \sum_{t=1}^{n} z_{t, n}^{*}\left(x, \boldsymbol{a}_{i}, \boldsymbol{b}_{i}\right) \mathbb{1}_{\underline{y_{t-1}} \in \mathcal{R}_{i}}\right|>u\right) \leq \frac{K}{n u^{4}}\left\{\left(x \sup _{i \in I}\left\|\boldsymbol{a}_{i}\right\|\right)^{2}+\sup _{i \in I}\left\|\boldsymbol{b}_{i}\right\|^{2}+1\right\} .
$$

Proof. We note that, for fixed $n$ and $x,\left(\tilde{z}_{t}, \mathcal{F}_{t}\right)_{1 \leq t \leq n}$ is a martingale difference sequence, where $\tilde{z}_{t}=\sum_{i \in I} z_{t, n}^{*}\left(x, \boldsymbol{a}_{i}, \boldsymbol{b}_{i}\right) \mathbb{1}_{y_{t-1} \in \mathcal{R}_{i}}$. Hence, by the Markov and Rosenthal inequalities

$$
P\left(\left|\frac{1}{\sqrt{n}} \sum_{t=1}^{n} \tilde{z}_{t}\right|>u\right) \leq \frac{1}{n^{2}} \frac{1}{u^{4}} E\left(\sum_{t=1}^{n} \tilde{z}_{t}\right)^{4} \leq \frac{K}{n^{2} u^{4}}\left\{E\left(\sum_{t=1}^{n} E\left(\tilde{z}_{t}^{2} \mid \mathcal{F}_{t-1}\right)\right)^{2}+\sum_{t=1}^{n} E \tilde{z}_{t}^{4}\right\} .
$$

We have

$$
\begin{aligned}
E\left(\tilde{z}_{t}^{2} \mid \mathcal{F}_{t-1}\right) & =\sum_{i \in I} E\left[z_{t, n}^{* 2}\left(x, \boldsymbol{a}_{i}, \boldsymbol{b}_{i}\right) \mid \mathcal{F}_{t-1}\right] \mathbb{1}_{\underline{\underline{t-1}} \in \mathcal{R}_{i}} \\
& \leq \sum_{i \in I}\left|F\left(x \gamma_{t, n}^{*}\left(\boldsymbol{a}_{i}\right)+\lambda_{t, n}^{*}\left(\boldsymbol{b}_{i}\right)\right)-F(x)\right| \mathbb{1}_{\underline{\underline{t-1}} \in \mathcal{R}_{i}} .
\end{aligned}
$$

By A11, letting $K=\sup _{y} f(y)<\infty$,

$$
\begin{aligned}
& \left|F\left(x \gamma_{t, n}^{*}\left(\boldsymbol{a}_{i}\right)+\lambda_{t, n}^{*}\left(\boldsymbol{b}_{i}\right)\right)-F(x)\right| \\
\leq & |x| K\left|\gamma_{t, n}^{*}\left(\boldsymbol{a}_{i}\right)-1\right|+K\left|\lambda_{t, n}^{*}\left(\boldsymbol{b}_{i}\right)\right| \\
\leq & \frac{K}{\sqrt{n}}\left\{|x|\left\|\frac{1}{\sigma_{t}\left(\boldsymbol{\theta}_{0}\right)} \frac{\partial \sigma_{t}\left(\boldsymbol{\theta}_{0}\right)}{\partial \boldsymbol{\theta}}\right\|\left\|\boldsymbol{a}_{i}\right\|+\left\|\frac{1}{\sigma_{t}\left(\boldsymbol{\theta}_{0}\right)} \frac{\partial m_{t}\left(\boldsymbol{\theta}_{0}\right)}{\partial \boldsymbol{\theta}}\right\|\left\|\boldsymbol{b}_{i}\right\|\right\} .
\end{aligned}
$$

The rest of the proof is similar to that of Lemma 2 .

Lemma 11. Let $\mathcal{K}$ a compact subset of $\mathbb{R}$. We have, for any sequence $\left(\boldsymbol{a}_{i}\right)_{i \in I}$ and $\left(\boldsymbol{b}_{i}\right)_{i \in I}$ such that $\sup _{i \in I}\left\|\boldsymbol{a}_{i}\right\|+\left\|\boldsymbol{b}_{i}\right\|<\infty$,

$$
\boldsymbol{\mathcal { X }}_{n}\left(\left(\boldsymbol{a}_{i}\right)_{i \in I},\left(\boldsymbol{b}_{i}\right)_{i \in I}\right):=\sup _{x \in \mathcal{K}}\left|\sum_{i \in I} \frac{1}{\sqrt{n}} \sum_{t=1}^{n} z_{t, n}^{*}\left(x, \boldsymbol{a}_{i}, \boldsymbol{b}_{i}\right) \mathbb{1}_{\underline{\underline{t-1}} \in \mathcal{R}_{i}}\right|=o_{P}(1) .
$$

Proof. Using Lemma 10, the proof is similar to that of Lemma 3. See Appendix.

Lemma 12. Let $\mathcal{K}$ be a compact subset of $\mathbb{R}$. We have, for any $A>0$ and $\boldsymbol{A}=[-A, A]^{d}$

$$
\sup _{x \in \mathcal{K}} \sup _{\boldsymbol{a} \in \boldsymbol{A}}\left|e_{n, 4}(x, \boldsymbol{a})-e_{n}(x)\right|=o_{P}(1) \text {. }
$$

Proof. Note that $e_{n, 4}(x, \boldsymbol{a})-e_{n}(x)=n^{-1 / 2} \sum_{t=1}^{n} z_{t, n}^{*}(x, \boldsymbol{a}, \boldsymbol{a})$. We will thus show that

$$
\sup _{\boldsymbol{a}, \boldsymbol{b} \in \boldsymbol{A}} X_{n}^{*}(\boldsymbol{a}, \boldsymbol{b})=o_{P}(1), \quad \text { where } \quad X_{n}^{*}(\boldsymbol{a}, \boldsymbol{b})=\sup _{x \in \mathcal{K}}\left|\frac{1}{\sqrt{n}} \sum_{t=1}^{n} z_{t, n}^{*}(x, \boldsymbol{a}, \boldsymbol{b})\right| .
$$


Let $\varepsilon>0$ such that $N:=2 A / \varepsilon$ is an integer and define $a(k)=-A+k \varepsilon$ for $1 \leq k \leq N$. For $1 \leq$ $k_{1}, k_{2}, \ldots k_{d} \leq N$, let $\boldsymbol{k}=\left(k_{1}, \ldots, k_{d}\right)$ and consider the grid of $N^{d}$ points $\boldsymbol{a}(\boldsymbol{k})=\left(a\left(k_{1}\right), \ldots, a\left(k_{d}\right)\right)$. Let also $\boldsymbol{A}(\boldsymbol{k})=\left\{\left(a_{1}, \ldots, a_{d}\right) \in \boldsymbol{A} \mid a\left(k_{j}\right)-\varepsilon \leq a_{j} \leq a\left(k_{j}\right), j \in\{1, \ldots, d\}\right\}$. Define, for $i \geq 1$, and $\boldsymbol{s}_{i}=\left(\boldsymbol{s}_{i}^{(1)}, \boldsymbol{s}_{i}^{(2)}\right)=\left(s_{i 1}^{(1)}, \ldots, s_{i d}^{(1)}, s_{i 1}^{(2)}, \ldots, s_{i d}^{(2)}\right)$

$$
\begin{aligned}
& \overline{\boldsymbol{a}}_{s_{i}^{(\ell)}}(\boldsymbol{k})=\left(\bar{a}_{s_{i 1}^{(\ell)}}\left(k_{1}\right), \ldots, \bar{a}_{s_{i d}^{(\ell)}}\left(k_{d}\right)\right), \quad \bar{a}_{s_{i j}^{(\ell)}}\left(k_{j}\right)=a\left(k_{j}\right) \mathbb{1}_{s_{i j}^{(\ell)}=1}+\left\{a\left(k_{j}\right)-\varepsilon\right\} \mathbb{1}_{s_{i j}^{(\ell)}=-1}, \\
& \underline{\boldsymbol{a}}_{s_{i}^{(\ell)}}(\boldsymbol{k})=\left(\underline{a}_{s_{i 1}^{(\ell)}}\left(k_{1}\right), \ldots, \underline{a}_{s_{i d}^{(\ell)}}\left(k_{d}\right)\right), \quad \underline{a}_{s_{i j}^{(\ell)}}\left(k_{j}\right)=a\left(k_{j}\right) \mathbb{1}_{s_{i j}^{(\ell)}=-1}+\left\{a\left(k_{j}\right)-\varepsilon\right\} \mathbb{1}_{s_{i j}^{(\ell)}=1} .
\end{aligned}
$$

We have, for $j=1, \ldots, d$,

$$
\begin{aligned}
& F\left(x \gamma_{t, n}^{*}(\boldsymbol{a})+\lambda_{t, n}^{*}\left(b_{1}, \ldots, b_{j-1}, a\left(k_{j}\right), b_{j+1}, \ldots, b_{d}\right)\right)-F\left(x \gamma_{t, n}^{*}(\boldsymbol{a})+\lambda_{t, n}^{*}(\boldsymbol{b})\right) \\
= & f\left\{x \gamma_{t, n}^{*}(\boldsymbol{a})+\lambda_{t, n}^{*}\left(\boldsymbol{b}_{t, j}\right)\right\} \frac{a\left(k_{j}\right)-b_{j}}{\sqrt{n}} \frac{1}{\sigma_{t}\left(\boldsymbol{\theta}_{0}\right)} \frac{\partial m_{t}\left(\boldsymbol{\theta}_{0}\right)}{\partial \boldsymbol{\theta}^{\prime}} \boldsymbol{e}_{j},
\end{aligned}
$$

where $\boldsymbol{e}_{j}$ is the $j$-th element of the canonical basis of $\mathbb{R}^{d}$, and $\boldsymbol{b}_{t, j}$ is a point between the arguments of $\lambda_{t, n}$ above. By A11, we have $\sup _{x} f(x)<\infty$. The absolute value of the latter difference is thus bounded, uniformly in $x \in \mathbb{R}, \boldsymbol{a} \in \boldsymbol{A}$ and $\boldsymbol{b} \in \boldsymbol{A}(\boldsymbol{k})$, by $\quad K \frac{\varepsilon}{\sqrt{n}} \frac{1}{\sigma_{t}\left(\boldsymbol{\theta}_{0}\right)}\left|\frac{\partial m_{t}\left(\boldsymbol{\theta}_{0}\right)}{\partial \boldsymbol{\theta}^{\prime}} \boldsymbol{e}_{j}\right|$.

Similarly, for $j=1, \ldots, d$,

$$
\begin{aligned}
& \left|F\left(x \gamma_{t, n}^{*}\left(a_{1}, \ldots, a_{j-1}, a\left(k_{j}\right), a_{j+1}, \ldots, a_{d}\right)+\lambda_{t, n}^{*}(\boldsymbol{b})\right)-F\left(x \gamma_{t, n}^{*}(\boldsymbol{a})+\lambda_{t, n}^{*}(\boldsymbol{b})\right)\right| \\
= & \left|x f\left\{x \gamma_{t, n}^{*}\left(\boldsymbol{a}_{t, j}\right)+\lambda_{t, n}^{*}(\boldsymbol{b})\right\} \frac{a\left(k_{j}\right)-a_{j}}{\sqrt{n}} \frac{1}{\sigma_{t}\left(\boldsymbol{\theta}_{0}\right)} \frac{\partial \sigma_{t}\left(\boldsymbol{\theta}_{0}\right)}{\partial \boldsymbol{\theta}^{\prime}} \boldsymbol{e}_{j}\right| \leq K \frac{\varepsilon}{\sqrt{n}} \frac{1}{\sigma_{t}\left(\boldsymbol{\theta}_{0}\right)}\left|\frac{\partial \sigma_{t}\left(\boldsymbol{\theta}_{0}\right)}{\partial \boldsymbol{\theta}^{\prime}} \boldsymbol{e}_{j}\right|,
\end{aligned}
$$

uniformly in $x \in \mathcal{K}, \boldsymbol{b} \in \boldsymbol{A}$ and $\boldsymbol{a} \in \boldsymbol{A}(\boldsymbol{k})$. Therefore, for $n$ large enough,

$$
\begin{aligned}
& \sup _{\boldsymbol{a} \in \boldsymbol{A}(\boldsymbol{k}), \boldsymbol{b} \in \boldsymbol{A}(\ell)} \sup _{x \in \mathcal{K}} \sum_{t=1}^{n}\left|F\left(x \gamma_{t, n}^{*}(\boldsymbol{a})+\lambda_{t, n}^{*}(\boldsymbol{b})\right)-F\left(x \gamma_{t, n}^{*}(\boldsymbol{a}(\boldsymbol{k}))+\lambda_{t, n}^{*}(\boldsymbol{b}(\ell))\right)\right| \\
\leq & K \frac{\varepsilon}{\sqrt{n}} \sum_{t=1}^{n}\left\|\frac{1}{\sigma_{t}\left(\boldsymbol{\theta}_{0}\right)} \frac{\partial \sigma_{t}\left(\boldsymbol{\theta}_{0}\right)}{\partial \boldsymbol{\theta}}\right\|+\left\|\frac{1}{\sigma_{t}\left(\boldsymbol{\theta}_{0}\right)} \frac{\partial m_{t}\left(\boldsymbol{\theta}_{0}\right)}{\partial \boldsymbol{\theta}}\right\|:=K \frac{\varepsilon}{\sqrt{n}} \sum_{t=1}^{n} Z_{t} .
\end{aligned}
$$

It can be shown (see Appendix) that

$$
\begin{aligned}
& \sup _{\boldsymbol{a} \in \boldsymbol{A}(\boldsymbol{k}), \boldsymbol{b} \in \boldsymbol{A}(\boldsymbol{\ell})} \sup _{x \in \mathcal{K}}\left|\frac{1}{\sqrt{n}} \sum_{t=1}^{n} z_{t, n}^{*}(x, \boldsymbol{a}, \boldsymbol{b})-z_{t, n}^{*}(x, \boldsymbol{a}(\boldsymbol{k}), \boldsymbol{a}(\boldsymbol{\ell}))\right| \\
\leq & \boldsymbol{\mathcal { X }}_{n}\left(\left(\overline{\boldsymbol{a}}_{s_{i}^{(2)}}(\boldsymbol{k})\right)_{i \in I},\left(\overline{\boldsymbol{a}}_{s_{i}^{(1)}}(\boldsymbol{\ell})\right)_{i \in I}\right)+\boldsymbol{\mathcal { X }}_{n}\left(\left(\underline{\boldsymbol{a}}_{s_{i}^{(2)}}(\boldsymbol{k})\right)_{i \in I},\left(\underline{\boldsymbol{a}}_{s_{i}^{(1)}}(\boldsymbol{\ell})\right)_{i \in I}\right) \\
+ & \boldsymbol{\mathcal { X }}_{n}\left(\left(\underline{\boldsymbol{a}}_{s_{i}^{(2)}}(\boldsymbol{k})\right)_{i \in I},\left(\overline{\boldsymbol{a}}_{s_{i}^{(1)}}(\boldsymbol{\ell})\right)_{i \in I}\right)+\boldsymbol{\mathcal { X }}_{n}\left(\left(\overline{\boldsymbol{a}}_{s_{i}^{(2)}}(\boldsymbol{k})\right)_{i \in I},\left(\underline{\boldsymbol{a}}_{s_{i}^{(1)}}(\boldsymbol{\ell})\right)_{i \in I}\right)+3 K \frac{\varepsilon}{n} \sum_{t=1}^{n} Z_{t} .
\end{aligned}
$$

Note that

$$
\sup _{\boldsymbol{a}, \boldsymbol{b} \in \boldsymbol{A}} X_{n}^{*}(\boldsymbol{a}, \boldsymbol{b})
$$




$$
\begin{aligned}
\leq & \max _{\boldsymbol{k}, \ell \in\{1, \ldots, N\}^{d}} \sup _{\boldsymbol{a} \in \boldsymbol{A}(\boldsymbol{k}), \boldsymbol{b} \in \boldsymbol{B}(\ell)} \sup _{x \in \mathcal{K}}\left|\frac{1}{\sqrt{n}} \sum_{t=1}^{n}\left[z_{t, n}^{*}(x, \boldsymbol{a}, \boldsymbol{b})-z_{t, n}^{*}(x, \boldsymbol{a}(\boldsymbol{k}), \boldsymbol{b}(\ell))\right]\right| \\
& +\max _{\boldsymbol{k}, \ell \in\{1, \ldots, N\}^{d}} X_{n}^{*}(\boldsymbol{a}(\boldsymbol{k}), \boldsymbol{a}(\boldsymbol{\ell})) \\
\leq & \frac{3 K \varepsilon}{n} \sum_{t=1}^{n} Z_{t}+5 \max _{\left(\boldsymbol{a}_{i}\right),\left(\boldsymbol{b}_{i}\right)} \boldsymbol{\mathcal { X }}_{n}\left(\left(\boldsymbol{a}_{i}\right)_{i \in I},\left(\boldsymbol{b}_{i}\right)_{i \in I}\right),
\end{aligned}
$$

where the last max is taken over all sequences of the form $\overline{\boldsymbol{a}}_{s_{i}^{(\ell)}}(\boldsymbol{k})$ or $\underline{\boldsymbol{a}}_{s_{i}^{(\ell)}}(\boldsymbol{k}), \boldsymbol{\ell} \in\{1,2\}$ and $\boldsymbol{k}, \boldsymbol{\ell} \in\{1, \ldots, N\}^{d}$. The end of the proof of (25) is similar to that of (21) using Lemma 11.

Lemma 13. Let $\mathcal{K}$ be a compact subset of $\mathbb{R}$. Then $\sup _{x \in \mathcal{K}}\left|\widehat{e}_{n, 5}(x)-H_{n}(x)\right| \rightarrow 0$ a.s.

Proof. A Taylor expansion yields, for $x_{t}^{*}$ between $x$ and $x \chi_{t, n}^{*}+\lambda_{t, n}^{*}$,

$$
F\left(x \chi_{t, n}^{*}+\lambda_{t, n}^{*}\right)-F(x)=f\left(x_{t}^{*}\right) \frac{1}{\sigma_{t}}\left(x \frac{\partial \sigma_{t}\left(\boldsymbol{\theta}_{0}\right)}{\partial \boldsymbol{\theta}^{\prime}}+\frac{\partial m_{t}\left(\boldsymbol{\theta}_{0}\right)}{\partial \boldsymbol{\theta}^{\prime}}\right)\left(\widehat{\boldsymbol{\theta}}_{n}-\boldsymbol{\theta}_{0}\right) .
$$

It follows that

$$
\begin{aligned}
\left|\widehat{e}_{n, 5}(x)-H_{n}(x)\right|= & \left|\frac{1}{\sqrt{n}} \sum_{t=1}^{n}\left\{f\left(x_{t}^{*}\right)-f(x)\right\} \frac{1}{\sigma_{t}}\left(x \frac{\partial \sigma_{t}\left(\boldsymbol{\theta}_{0}\right)}{\partial \boldsymbol{\theta}^{\prime}}+\frac{\partial m_{t}\left(\boldsymbol{\theta}_{0}\right)}{\partial \boldsymbol{\theta}^{\prime}}\right)\left(\widehat{\boldsymbol{\theta}}_{n}-\boldsymbol{\theta}_{0}\right)\right| \\
\leq & \left(\frac{1}{n} \sum_{t=1}^{n}\left|f\left(x_{t}^{*}\right)-f(x)\right|^{2}\right)^{1 / 2} \\
& \times\left(\frac{1}{n} \sum_{t=1}^{n}|x|^{2}\left\{\left\|\frac{1}{\sigma_{t}} \frac{\partial \sigma_{t}\left(\boldsymbol{\theta}_{0}\right)}{\partial \boldsymbol{\theta}}\right\|+\left\|\frac{1}{\sigma_{t}} \frac{\partial m_{t}\left(\boldsymbol{\theta}_{0}\right)}{\partial \boldsymbol{\theta}}\right\|\right\}^{2}\right)^{1 / 2}\left\|\sqrt{n}\left(\widehat{\boldsymbol{\theta}}_{n}-\boldsymbol{\theta}_{0}\right)\right\|
\end{aligned}
$$

by Cauchy-Schwarz inequality. We have, for any $\Delta>0$ and $n$ large enough

$$
\begin{aligned}
& \frac{1}{n} \sum_{t=1}^{n} \sup _{x \in \mathcal{K}}\left|f\left(x_{t}^{*}\right)-f(x)\right|^{2} \\
\leq & \frac{1}{n} \sum_{t=1}^{n} \sup _{\boldsymbol{\delta}_{\boldsymbol{\theta}}:\left\|\boldsymbol{\delta}_{\boldsymbol{\theta}}\right\| \leq \Delta} \sup _{x \in \mathcal{K}}\left|f\left(x+\frac{1}{\sigma_{t}}\left(x \frac{\partial \sigma_{t}\left(\boldsymbol{\theta}_{0}\right)}{\partial \boldsymbol{\theta}^{\prime}}+\frac{\partial m_{t}\left(\boldsymbol{\theta}_{0}\right)}{\partial \boldsymbol{\theta}^{\prime}}\right) \boldsymbol{\delta}_{\boldsymbol{\theta}}\right)-f(x)\right|^{2} \\
& +\mathbb{1}_{\left\|\widehat{\boldsymbol{\theta}}_{n}-\boldsymbol{\theta}_{0}\right\|>\Delta}
\end{aligned}
$$

The first term in the r.h.s. converges a.s. to

$$
E \sup _{\boldsymbol{\delta}_{\boldsymbol{\theta}}:\left\|\boldsymbol{\delta}_{\boldsymbol{\theta}}\right\| \leq \Delta} \sup _{x \in \mathcal{K}}\left|f\left(x+\frac{1}{\sigma_{t}}\left(x \frac{\partial \sigma_{t}\left(\boldsymbol{\theta}_{0}\right)}{\partial \boldsymbol{\theta}^{\prime}}+\frac{\partial m_{t}\left(\boldsymbol{\theta}_{0}\right)}{\partial \boldsymbol{\theta}^{\prime}}\right) \boldsymbol{\delta}_{\boldsymbol{\theta}}\right)-f(x)\right|^{2}
$$

which can be made arbitrarily small for $\Delta$ small enough by the dominated convergence theorem. The last term in the r.h.s. is a.s. equal to zero for $n$ large enough. The conclusion follows.

Write

$$
\widehat{e}_{n, 6}(x)=\underbrace{\frac{1}{\sqrt{n}} \sum_{t=1}^{n} \mathbb{1}_{\eta_{t} \leq x \tilde{\chi}_{t, n}+\tilde{\lambda}_{t, n}}-\mathbb{1}_{\eta_{t} \leq x \chi_{t, n}+\lambda_{t, n}}}_{\widehat{e}_{n, 7}(x)}+\underbrace{\frac{1}{\sqrt{n}} \sum_{t=1}^{n} \mathbb{1}_{\eta_{t} \leq x \chi_{t, n}+\lambda_{t, n}}-\mathbb{1}_{\eta_{t} \leq x \chi_{t, n}^{*}+\lambda_{t, n}^{*}}}_{\widehat{e}_{n, 8}(x)} .
$$


Lemma 14. We have $\left|\widehat{e}_{n, 6}\left(x_{n}\right)\right|=o_{P}(1)$.

Proof. We prove in the Appendix that $\sup _{x \in \mathbb{R}}\left|\widehat{e}_{n, 7}(x)\right|=o_{P}(1)$. From Taylor expansions we get

$$
\begin{aligned}
& \left|x \chi_{t, n}+\lambda_{t, n}-\left(x \chi_{t, n}^{*}+\lambda_{t, n}^{*}\right)\right| \\
\leq & \frac{2}{\sigma_{t}\left(\boldsymbol{\theta}_{0}\right)} \sup _{\boldsymbol{\theta} \in V\left(\boldsymbol{\theta}_{0}\right)}\left\{|x|\left\|\frac{\partial^{2} \sigma_{t}(\boldsymbol{\theta})}{\partial \boldsymbol{\theta} \partial \boldsymbol{\theta}^{\prime}}\right\|+\left\|\frac{\partial^{2} m_{t}(\boldsymbol{\theta})}{\partial \boldsymbol{\theta} \partial \boldsymbol{\theta}^{\prime}}\right\|\right\}\left\|\widehat{\boldsymbol{\theta}}_{n}-\boldsymbol{\theta}_{0}\right\|^{2}:=\delta_{t}(x)\left\|\widehat{\boldsymbol{\theta}}_{n}-\boldsymbol{\theta}_{0}\right\|^{2},
\end{aligned}
$$

where $\boldsymbol{\theta}_{t}, \underline{\boldsymbol{\theta}}_{t}$ and $\overline{\boldsymbol{\theta}}_{t}$ are between $\boldsymbol{\theta}_{0}$ and $\widehat{\boldsymbol{\theta}}_{n}$. It follows that, for any $\Delta_{n}>0$,

$$
\left|\widehat{e}_{n, 8}\left(x_{n}\right)\right| \leq \frac{1}{\sqrt{n}} \sum_{t=1}^{n} \mathbb{1}_{x_{n} \chi_{t, n}^{*}+\lambda_{t, n}^{*}-\delta_{t}\left(x_{n}\right) \Delta_{n}^{2} \leq \eta_{t} \leq x_{n} \chi_{t, n}^{*}+\lambda_{t, n}^{*}+\delta_{t}\left(x_{n}\right) \Delta_{n}^{2}}+\sqrt{n} \mathbb{1}_{\left\|\widehat{\boldsymbol{\theta}}_{n}-\boldsymbol{\theta}_{0}\right\|>\Delta_{n}} .
$$

Let $\Delta_{n}=n^{s-1 / 2}$, for some $s>0$. The last term in (26) is equal to $c_{4, n}=0$ for sufficiently large $n$. Now, first suppose that the sequence $\left(x_{n}\right)$ is non random, $x_{n} \rightarrow x$. Note that the sequence $\left(\delta_{t}\left(x_{n}\right)\right)_{t}$ is stationary, and integrable by Assumptions A9 and B7. Let $f_{t, n}$ the conditional density of $\eta_{t}$ introduced in Assumption B8, and let $K$ its uniform upper bound. Then, by the law of iterated expectations, the expectation of the first term in (26) is bounded, for $n>n_{0}$, by

$$
2 \sqrt{n} \Delta_{n}^{2} K E\left\{\delta_{1}\left(x_{n}\right)\right\}+\frac{t(n)}{\sqrt{n}}=o(1) .
$$

The conclusion follows in case i). Turning to case ii), using Lemma 4.4 in Kreiss (1987), we can deduce that the conclusion also holds.

Lemma 15. Let $\mathcal{K}$ be a compact subset of $\mathbb{R}$. We have $\sup _{x \in \mathcal{K}}\left|\widehat{e}_{n}(x)-e_{n}(x)-H_{n}(x)\right|=o_{P}(1)$.

Proof. In view of $(24),\left|\widehat{e}_{n}(x)-e_{n}(x)-H_{n}(x)\right| \leq\left|\widehat{e}_{n, 4}(x)-e_{n}(x)\right|+\left|\widehat{e}_{n, 5}(x)-H_{n}(x)\right|+\left|\widehat{e}_{n, 6}(x)\right|$. Note that $\widehat{e}_{n, 4}(x)=e_{n, 4}\left(x, \sqrt{n}\left(\widehat{\boldsymbol{\theta}}_{n}-\boldsymbol{\theta}_{0}\right)\right)$. Thus, any $u, A>0, \boldsymbol{A}=[-A, A]^{d}$,

$$
\begin{aligned}
P\left(\sup _{x \in \mathcal{K}}\left|\widehat{e}_{n, 4}(x)-e_{n}(x)\right|>u\right) \leq & P\left(\sup _{x \in \mathcal{K}} \sup _{\boldsymbol{a} \in \boldsymbol{A}}\left|e_{n, 4}(x, \boldsymbol{a})-e_{n}(x)\right|>u\right) \\
& +P\left(\sqrt{n}\left(\widehat{\boldsymbol{\theta}}_{n}-\boldsymbol{\theta}_{0}\right) \notin \boldsymbol{A}\right) .
\end{aligned}
$$

The conclusion follows as in Lemma 8, using lemmas 12, 13 and 14.

To complete the proof of Theorem 4, note that, by arguments used to prove Theorem 2, we have $\widehat{e}_{n}\left(x_{n}\right)=\frac{1}{\sqrt{n}} \sum_{t=1}^{n}\left\{\mathbb{1}_{\eta_{t}<x}-F(x)\right\}+f(x)\left(\boldsymbol{\Omega}_{m}^{\prime}+x \boldsymbol{\Omega}_{\sigma}^{\prime}\right) \frac{1}{\sqrt{n}} \sum_{t=1}^{n} \boldsymbol{\Delta}_{t-1} \boldsymbol{V}\left(\eta_{t}\right)+o_{P}(1)$. 


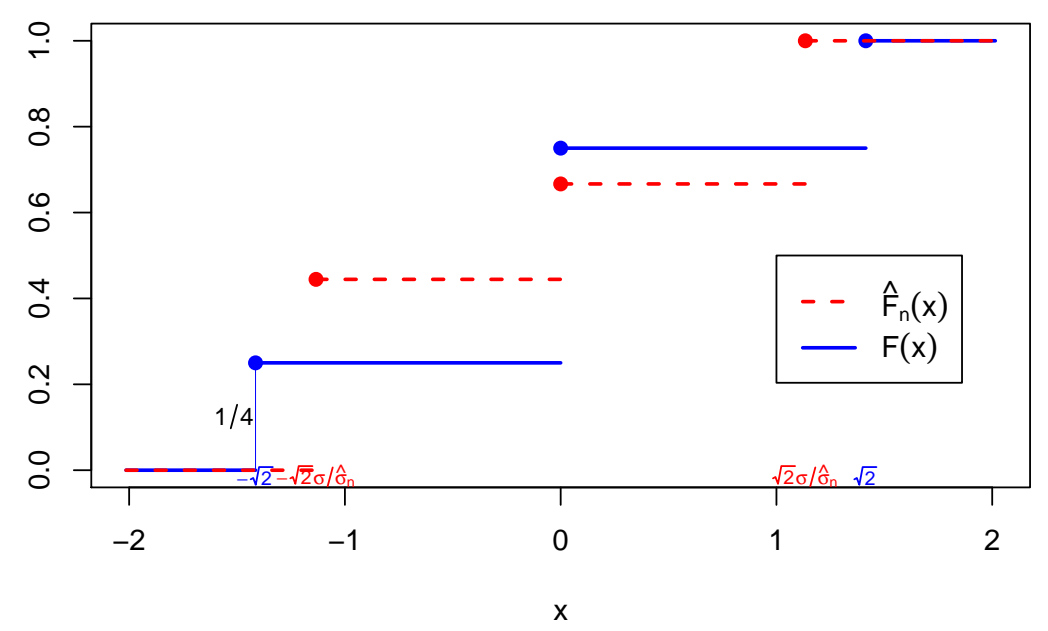

Fig. 1. Failure of the Glivenko-Cantelli property for residuals when A5 does not hold (Example 1).

\section{Appendix}

\section{A. Illustration of Example 1 and Remark 1}

A graph illustrating the discrepancy between $\widehat{F}_{n}(x)$ and $F(x)$ is displayed in Figure 1.

A graph illustrating the effect of the estimation on the asymptotic distribution of the e.d.f.r in displayed in Figure 2.

\section{B. Proof of Theorem 3}

The proof requires the following changes in the proof of Theorem 1. Let $\eta_{t}(\boldsymbol{\theta})=\left(y_{t}-m_{t}(\boldsymbol{\theta})\right) / \sigma_{t}(\boldsymbol{\theta})$ and $\tilde{\eta}_{t}(\boldsymbol{\theta})=\left(y_{t}-\tilde{m}_{t}(\boldsymbol{\theta})\right) / \tilde{\sigma}_{t}(\boldsymbol{\theta})$. We have $\sup _{\boldsymbol{\theta} \in \boldsymbol{\Theta}}\left|\eta_{t}(\boldsymbol{\theta})-\tilde{\eta}_{t}(\boldsymbol{\theta})\right| \leq K_{t} \rho^{t}\left(1+\left|y_{t}\right|\right)$. Thus, by a Taylor expansion,

$$
\left|\widehat{\eta}_{t}-\eta_{t}\right| \leq\left\|\widehat{\boldsymbol{\theta}}_{n}-\boldsymbol{\theta}_{0}\right\| x_{t}+K_{t} \rho^{t}\left(1+\left|y_{t}\right|\right)
$$

for $n$ large enough, where

$$
x_{t}=\left\{\left|\eta_{t}\right| \sup _{\boldsymbol{\theta} \in V\left(\boldsymbol{\theta}_{0}\right)} \frac{\sigma_{t}\left(\boldsymbol{\theta}_{0}\right)}{\sigma_{t}(\boldsymbol{\theta})}+\sup _{\boldsymbol{\theta} \in V\left(\boldsymbol{\theta}_{0}\right)} \frac{\left|m_{t}\left(\boldsymbol{\theta}_{0}\right)-m_{t}(\boldsymbol{\theta})\right|}{\sigma_{t}(\boldsymbol{\theta})}\right\} \sup _{\boldsymbol{\theta} \in V\left(\boldsymbol{\theta}_{0}\right)}\left\|\boldsymbol{D}_{t}(\boldsymbol{\theta})\right\|+\sup _{\boldsymbol{\theta} \in V\left(\boldsymbol{\theta}_{0}\right)} \frac{1}{\sigma_{t}(\boldsymbol{\theta})}\left\|\frac{\partial m_{t}(\boldsymbol{\theta})}{\partial \boldsymbol{\theta}}\right\| .
$$

In view of (27)-(28), we have

$$
\left|\widehat{\eta}_{t}-\eta_{t}\right| \leq K_{t} \rho^{t}\left(1+\left|y_{t}\right|\right)+\left(u_{t}\left|\eta_{t}\right|+v_{t}\right)\left\|\widehat{\boldsymbol{\theta}}_{n}-\boldsymbol{\theta}_{0}\right\|
$$



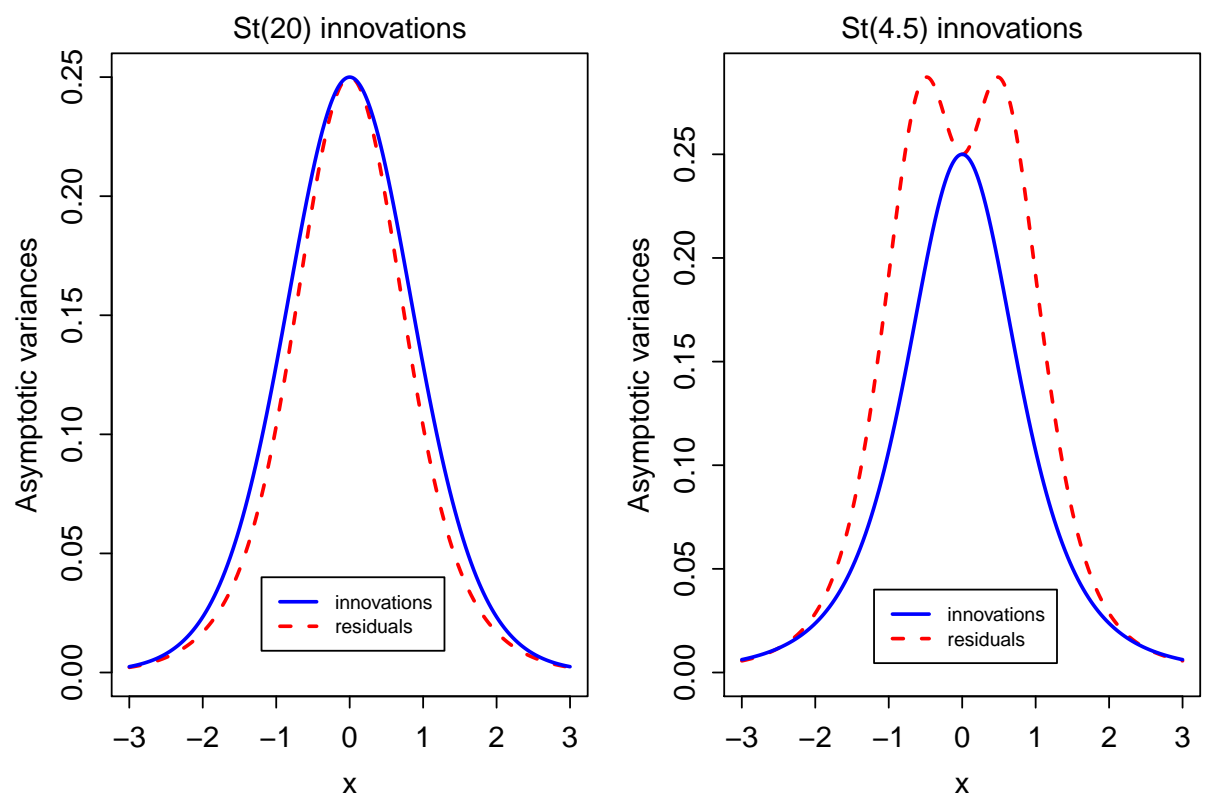

Fig. 2. Asymptotic variances of the empirical distribution for residuals (dotted red lines) and innovations (full blue line), for standardized Gaussian, GED (Generalized Error Distribution) and Student distributions.

for $n$ large enough, where $u_{t}, v_{t} \in \mathcal{F}_{t-1}$ and, by $\mathbf{B 4}, E\left(\left|u_{t}\right|^{r / 2}\right)<\infty$ and $E\left(\left|v_{t}\right|^{r / 2}\right)<\infty$. The rest of the proof of the Glivenko-Cantelli property is similar to that of Theorem 1, using the consistency of $\widehat{\boldsymbol{\theta}}_{n}$.

\section{Complements to Theorem 4}

The next two results avoid the discreteness of the estimator in Theorem 4. Instead, we first assume faster convergence of the sequence $\left(x_{n}\right)$.

Proposition 1. Let $x \in \mathbb{R}$ and let $\left(x_{n}\right)$ a sequence of random variables such that $\sqrt{n}\left(x_{n}-x\right)=$ o(1) a.s. Then, under B1-B8 and A9, A11, the conclusions of Theorem 4 hold.

Proof. The proof is the same as that of Theorem 4, except for Lemma 14. Let $\varepsilon>0, \underline{x}_{n}=$ $x-n^{-1 / 2} \varepsilon$, and $\bar{x}_{n}=x+n^{-1 / 2} \varepsilon$. Then, by the law of iterated expectations, the expectation of the first term in (26) is bounded, for $n$ large enough, by

$$
\sqrt{n}\left(\bar{x}_{n}-\underline{x}_{n}\right) E\left(\chi_{1, n}^{*}\right)+2 \sqrt{n} \Delta_{n}^{2} K E\left\{\delta_{1}\left(x_{n}\right)\right\}=2 \varepsilon+o(1)
$$

Because $\varepsilon$ can be chosen arbitrarily small, the conclusion follows.

In our second result, discreteness is avoided at the price of reinforcing Assumption B8. For any sequence $\left(x_{n}\right)$ of random variables, let the assumption 
B8 $\left(x_{n}\right)$ : For $n$ large enough, $n>n_{0}$ say, the conditional distribution of $\eta_{t}$ given $\left(\widehat{\boldsymbol{\theta}}_{n}, x_{n}, \mathcal{F}_{t-1}\right)$ admits a density which is a.s. bounded, uniformly in $n>n_{0}$ and $t<n-t(n)$ where $t(n)=$ $o(\sqrt{n})$.

Proposition 2. Let $x \in \mathbb{R}$ and let $\left(x_{n}\right)$ a sequence of random variables such that $x_{n} \rightarrow x$ in probability. Then, under $\mathbf{B 1 - B 7 ,} \mathbf{B 8}\left(x_{n}\right)$ and $\mathbf{A 9}$, A11, the conclusions of Theorem 4 hold.

Proof. Again, we just change the proof of Lemma 14. Noting that, for $n>n_{0}$ and $t<n-t(n)$

$$
E\left(\mathbb{1}_{x_{n} \chi_{t, n}^{*}+\lambda_{t, n}^{*}-\delta_{t}\left(x_{n}\right) \Delta_{n}^{2} \leq \eta_{t} \leq x_{n} \chi_{t, n}^{*}+\lambda_{t, n}^{*}+\delta_{t}\left(x_{n}\right) \Delta_{n}^{2}} \mid \widehat{\boldsymbol{\theta}}_{n}, x_{n}, \mathcal{F}_{t-1}\right)=2 K \delta_{t}\left(x_{n}\right) \Delta_{n}^{2},
$$

the conclusion follows.

Noting that there is no guarantee that $\gamma_{t, n}^{*}(\boldsymbol{a})$ be almost surely positive, we define

$$
\gamma_{t, n}^{* *}(\boldsymbol{a})=\left|\gamma_{t, n}^{*}(\boldsymbol{a})\right|
$$

Before showing Lemma 11, we need to slightly modify Lemma 10. Let

$$
z_{t, n}^{* *}(x, \boldsymbol{a}, \boldsymbol{b})=\mathbb{1}_{\eta_{t} \leq x \gamma_{t, n}^{* *}(\boldsymbol{a})+\lambda_{t, n}^{*}(\boldsymbol{b})}-F\left(x \gamma_{t, n}^{* *}(\boldsymbol{a})+\lambda_{t, n}^{*}(\boldsymbol{b})\right)-\left\{\mathbb{1}_{\eta_{t} \leq x}-F(x)\right\} .
$$

Lemma 16. We have, for any $u>0$ and sufficiently large $n$, for any sequences $\left(\boldsymbol{a}_{i}\right)_{i \in I}$ and $\left(\boldsymbol{b}_{i}\right)_{i \in I}$

$$
P\left(\left|\sum_{i \in I} \frac{1}{\sqrt{n}} \sum_{t=1}^{n} z_{t, n}^{* *}\left(x, \boldsymbol{a}_{i}, \boldsymbol{b}_{i}\right) \mathbb{1}_{\underline{\underline{t-1}} \in \mathcal{R}_{i}}\right|>u\right) \leq \frac{K}{n u^{4}}\left\{\left(x \sup _{i \in I}\left\|\boldsymbol{a}_{i}\right\|\right)^{2}+\sup _{i \in I}\left\|\boldsymbol{b}_{i}\right\|^{2}+1\right\} .
$$

Proof. Let $\check{z}_{t}=\sum_{i \in I} z_{t, n}^{* *}\left(x, \boldsymbol{a}_{i}, \boldsymbol{b}_{i}\right) \mathbb{1}_{y_{\underline{t-1}} \in \mathcal{R}_{i}}$. Note that, for fixed $n$ and $x,\left(\check{z}_{t}, \mathcal{F}_{t}\right)_{1 \leq t \leq n}$ is also a martingale difference sequence. Hence, we conclude by the arguments of the proof of Lemma 10 and

$$
\begin{aligned}
\left|F\left(x \gamma_{t, n}^{* *}\left(\boldsymbol{a}_{i}\right)+\lambda_{t, n}^{*}\left(\boldsymbol{b}_{i}\right)\right)-F(x)\right| & \leq|x| K\left|\gamma_{t, n}^{* *}\left(\boldsymbol{a}_{i}\right)-1\right|+K\left|\lambda_{t, n}^{*}\left(\boldsymbol{b}_{i}\right)\right| \\
& \leq|x| K\left|\gamma_{t, n}^{*}\left(\boldsymbol{a}_{i}\right)-1\right|+K\left|\lambda_{t, n}^{*}\left(\boldsymbol{b}_{i}\right)\right|
\end{aligned}
$$

using the elementary inequality ||$a|-| b|| \leq|a-| b||$.

Proof of Lemma 11. With the notations of Lemma 3, Lemma 10 and Lemma 16 entail that for any $u>0$,

$$
\begin{aligned}
& P\left(\max _{-N \leq j \leq N}\left|\sum_{i \in I} \frac{1}{\sqrt{n}} \sum_{t=1}^{n} z_{t, n}^{*}\left(x_{j}, \boldsymbol{a}_{i}, \boldsymbol{b}_{i}\right) \mathbb{1}_{\underline{\underline{t-1}} \in \mathcal{R}_{i}}\right|>u\right) \leq \frac{K}{\sqrt{n}}, \\
& P\left(\max _{-N \leq j \leq N}\left|\sum_{i \in I} \frac{1}{\sqrt{n}} \sum_{t=1}^{n} z_{t, n}^{* *}\left(x_{j}, \boldsymbol{a}_{i}, \boldsymbol{b}_{i}\right) \mathbb{1}_{\underline{y_{t-1}} \in \mathcal{R}_{i}}\right|>u\right) \leq \frac{K}{\sqrt{n}} .
\end{aligned}
$$


As argued in the proof of Lemma 3, using (30) it remains to show that

$$
\max _{-N \leq j \leq N-1} \sup _{x \in\left[x_{j}, x_{j+1}\right]}\left|\sum_{i \in I} \frac{1}{\sqrt{n}} \sum_{t=1}^{n}\left\{z_{t, n}^{*}\left(x, \boldsymbol{a}_{i}, \boldsymbol{b}_{i}\right)-z_{t, n}^{*}\left(x_{j}, \boldsymbol{a}_{i}, \boldsymbol{b}_{i}\right)\right\} \mathbb{1}_{\underline{y_{t-1}} \in \mathcal{R}_{i}}\right|=o_{P}(1) .
$$

For all $j$, we have

$$
\sum_{i \in I} \frac{1}{\sqrt{n}} \sum_{t=1}^{n}\left\{z_{t, n}^{*}\left(x, \boldsymbol{a}_{i}, \boldsymbol{b}_{i}\right)-z_{t, n}^{*}\left(x_{j}, \boldsymbol{a}_{i}, \boldsymbol{b}_{i}\right)\right\} \mathbb{1}_{\underline{\underline{t-1}} \in \mathcal{R}_{i}}=a_{n}(x)+b_{n}(x),
$$

with

$$
\begin{aligned}
a_{n}(x)=\sum_{i \in I} & \frac{1}{\sqrt{n}} \sum_{t=1}^{n}\left\{\mathbb{1}_{\eta_{t} \leq x \gamma_{t, n}^{*}\left(\boldsymbol{a}_{i}\right)+\lambda_{t, n}^{*}\left(\boldsymbol{b}_{i}\right)}-F\left(x \gamma_{t, n}^{*}\left(\boldsymbol{a}_{i}\right)+\lambda_{t, n}^{*}\left(\boldsymbol{b}_{i}\right)\right)\right. \\
-\mathbb{1}_{\eta_{t} \leq x_{j}} \gamma_{t, n}^{*}\left(\boldsymbol{a}_{i}\right)+\lambda_{t, n}^{*}\left(\boldsymbol{b}_{i}\right) & \left.+F\left(x_{j} \gamma_{t, n}^{*}\left(\boldsymbol{a}_{i}\right)+\lambda_{t, n}^{*}\left(\boldsymbol{b}_{i}\right)\right)\right\} \mathbb{1}_{\underline{\underline{t-1}} \in \mathcal{R}_{i}}
\end{aligned}
$$

and

$$
b_{n}(x)=\frac{1}{\sqrt{n}} \sum_{t=1}^{n}\left\{\mathbb{1}_{\eta_{t} \leq x}-F(x)-\mathbb{1}_{\eta_{t} \leq x_{j}}+F\left(x_{j}\right)\right\} .
$$

By (17) and (19), $\max _{-N \leq j \leq N-1} \sup _{x \in\left[x_{j}, x_{j+1}\right]}\left|b_{n}(x)\right|=o_{P}(1)$. For $j=0, \ldots, N-1$,

$$
\begin{aligned}
& \sup _{x \in\left[x_{j}, x_{j+1}\right]}\left|a_{n}(x)\right| \\
& \leq \frac{1}{\sqrt{n}} \sum_{i \in I} \sum_{t=1}^{n}\left\{\mathbb{1}_{\eta_{t} \leq x_{j+1} \gamma_{t, n}^{*}\left(\boldsymbol{a}_{i}\right)+\lambda_{t, n}^{*}\left(\boldsymbol{b}_{i}\right)}-\mathbb{1}_{\eta_{t} \leq x_{j} \gamma_{t, n}^{*}\left(\boldsymbol{a}_{i}\right)+\lambda_{t, n}^{*}\left(\boldsymbol{b}_{i}\right)}\right\} \mathbb{1}_{\gamma_{t, n}^{*}\left(\boldsymbol{a}_{i}\right) \geq 0} \mathbb{1}_{y_{\underline{t-1}} \in \mathcal{R}_{i}} \\
& +\frac{1}{\sqrt{n}} \sum_{i \in I} \sum_{t=1}^{n}\left\{\mathbb{1}_{\eta_{t} \leq x_{j} \gamma_{t, n}^{*}\left(\boldsymbol{a}_{i}\right)+\lambda_{t, n}^{*}\left(\boldsymbol{b}_{i}\right)}-\mathbb{1}_{\eta_{t} \leq x_{j+1} \gamma_{t, n}^{*}\left(\boldsymbol{a}_{i}\right)+\lambda_{t, n}^{*}\left(\boldsymbol{b}_{i}\right)}\right\} \mathbb{1}_{\gamma_{t, n}^{*}\left(\boldsymbol{a}_{i}\right)<0} \mathbb{1}_{\underline{\underline{t-1}} \in \mathcal{R}_{i}} \\
& +\frac{1}{\sqrt{n}} \sum_{i \in I} \sum_{t=1}^{n}\left\{F\left(x_{j+1} \gamma_{t, n}^{*}\left(\boldsymbol{a}_{i}\right)+\lambda_{t, n}^{*}\left(\boldsymbol{b}_{i}\right)\right)-F\left(x_{j} \gamma_{t, n}^{*}\left(\boldsymbol{a}_{i}\right)+\lambda_{t, n}^{*}\left(\boldsymbol{b}_{i}\right)\right)\right\} \mathbb{1}_{\gamma_{t, n}^{*}\left(\boldsymbol{a}_{i}\right) \geq 0} \mathbb{1}_{\underline{y_{t-1}} \in \mathcal{R}_{i}} \\
& +\frac{1}{\sqrt{n}} \sum_{i \in I} \sum_{t=1}^{n}\left\{F\left(x_{j} \gamma_{t, n}^{*}\left(\boldsymbol{a}_{i}\right)+\lambda_{t, n}^{*}\left(\boldsymbol{b}_{i}\right)\right)-F\left(x_{j+1} \gamma_{t, n}^{*}\left(\boldsymbol{a}_{i}\right)+\lambda_{t, n}^{*}\left(\boldsymbol{b}_{i}\right)\right)\right\} \mathbb{1}_{\gamma_{t, n}^{*}\left(\boldsymbol{a}_{i}\right)<0} \mathbb{1}_{\underline{y_{t-1}} \in \mathcal{R}_{i}} \\
& \leq \frac{1}{\sqrt{n}} \sum_{i \in I} \sum_{t=1}^{n}\left\{\mathbb{1}_{\eta_{t} \leq x_{j+1} \gamma_{t, n}^{* *}\left(\boldsymbol{a}_{i}\right)+\lambda_{t, n}^{*}\left(\boldsymbol{b}_{i}\right)}-\mathbb{1}_{\eta_{t} \leq x_{j} \gamma_{t, n}^{* *}\left(\boldsymbol{a}_{i}\right)+\lambda_{t, n}^{*}\left(\boldsymbol{b}_{i}\right)}\right\} \mathbb{1}_{\underline{y_{t-1}} \in \mathcal{R}_{i}} \\
& +\frac{1}{\sqrt{n}} \sum_{i \in I} \sum_{t=1}^{n}\left\{\mathbb{1}_{\eta_{t} \leq-x_{j} \gamma_{t, n}^{*}\left(\boldsymbol{a}_{i}\right)+\lambda_{t, n}^{*}\left(\boldsymbol{b}_{i}\right)}-\mathbb{1}_{\eta_{t} \leq-x_{j+1} \gamma_{t, n}^{*}\left(\boldsymbol{a}_{i}\right)+\lambda_{t, n}^{*}\left(\boldsymbol{b}_{i}\right)}\right\} \mathbb{1}_{\underline{\underline{t-1}} \in \mathcal{R}_{i}} \\
& +\frac{1}{\sqrt{n}} \sum_{i \in I} \sum_{t=1}^{n}\left\{F\left(x_{j+1} \gamma_{t, n}^{* *}\left(\boldsymbol{a}_{i}\right)+\lambda_{t, n}^{*}\left(\boldsymbol{b}_{i}\right)\right)-F\left(x_{j} \gamma_{t, n}^{* *}\left(\boldsymbol{a}_{i}\right)+\lambda_{t, n}^{*}\left(\boldsymbol{b}_{i}\right)\right)\right\} \mathbb{1}_{\underline{\underline{t-1}}} \in \mathcal{R}_{i} \\
& +\frac{1}{\sqrt{n}} \sum_{i \in I} \sum_{t=1}^{n}\left\{F\left(-x_{j} \gamma_{t, n}^{* *}\left(\boldsymbol{a}_{i}\right)+\lambda_{t, n}^{*}\left(\boldsymbol{b}_{i}\right)\right)-F\left(-x_{j+1} \gamma_{t, n}^{* *}\left(\boldsymbol{a}_{i}\right)+\lambda_{t, n}^{*}\left(\boldsymbol{b}_{i}\right)\right)\right\} \mathbb{1}_{\underline{\underline{t-1}} \in \mathcal{R}_{i}} \\
& \leq\left|\sum_{i \in I} \frac{1}{\sqrt{n}} \sum_{t=1}^{n} z_{t, n}^{* *}\left(x_{j+1}, \boldsymbol{a}_{i}, \boldsymbol{b}_{i}\right) \mathbb{1}_{\underline{\underline{t-1}} \in \mathcal{R}_{i}}\right|+\left|\sum_{i \in I} \frac{1}{\sqrt{n}} \sum_{t=1}^{n} z_{t, n}^{* *}\left(x_{j}, \boldsymbol{a}_{i}, \boldsymbol{b}_{i}\right) \mathbb{1}_{\underline{\underline{t-1}} \in \mathcal{R}_{i}}\right|
\end{aligned}
$$




$$
\begin{aligned}
& +\left|\sum_{i \in I} \frac{1}{\sqrt{n}} \sum_{t=1}^{n} z_{t, n}^{* *}\left(-x_{j+1}, \boldsymbol{a}_{i}, \boldsymbol{b}_{i}\right) \mathbb{1}_{\underline{\underline{t-1}} \in \mathcal{R}_{i}}\right|+\left|\sum_{i \in I} \frac{1}{\sqrt{n}} \sum_{t=1}^{n} z_{t, n}^{* *}\left(-x_{j}, \boldsymbol{a}_{i}, \boldsymbol{b}_{i}\right) \mathbb{1}_{\underline{\underline{t-1}} \in \mathcal{R}_{i}}\right| \\
& +V_{n}^{*}(j)+2 W_{n}^{*}\left(j, \boldsymbol{a}_{i}, \boldsymbol{b}_{i}\right),
\end{aligned}
$$

where

$$
\begin{aligned}
W_{n}^{*}\left(j, \boldsymbol{a}_{i}, \boldsymbol{b}_{i}\right)= & \frac{1}{\sqrt{n}} \sum_{i \in I} \sum_{t=1}^{n}\left\{F\left(x_{j+1} \gamma_{t, n}^{* *}\left(\boldsymbol{a}_{i}\right)+\lambda_{t, n}^{*}\left(\boldsymbol{b}_{i}\right)\right)-F\left(x_{j} \gamma_{t, n}^{* *}\left(\boldsymbol{a}_{i}\right)+\lambda_{t, n}^{*}\left(\boldsymbol{b}_{i}\right)\right)\right. \\
& \left.+F\left(-x_{j} \gamma_{t, n}^{* *}\left(\boldsymbol{a}_{i}\right)+\lambda_{t, n}^{*}\left(\boldsymbol{b}_{i}\right)\right)-F\left(-x_{j+1} \gamma_{t, n}^{* *}\left(\boldsymbol{a}_{i}\right)+\lambda_{t, n}^{*}\left(\boldsymbol{b}_{i}\right)\right)\right\} \mathbb{1}_{y_{\underline{t-1}} \in \mathcal{R}_{i}}, \\
V_{n}^{*}(j)= & n^{-1 / 2} \mid \sum_{t=1}^{n}\left\{\mathbb{1}_{\eta_{t} \leq x_{j+1}}-F\left(x_{j+1}\right)\right\}-\left\{\mathbb{1}_{\eta_{t} \leq x_{j}}-F\left(x_{j}\right)\right\} \\
& +\left\{\mathbb{1}_{\eta_{t} \leq-x_{j}}-F\left(-x_{j}\right)\right\}-\left\{\mathbb{1}_{\eta_{t} \leq-x_{j+1}}-F\left(-x_{j+1}\right)\right\} \mid .
\end{aligned}
$$

Thus (32) is established using (31) and the arguments of the proof of Lemma 3.

Detailed proof of Lemma 12. Note that $e_{n, 4}(x, \boldsymbol{a})-e_{n}(x)=n^{-1 / 2} \sum_{t=1}^{n} z_{t, n}^{*}(x, \boldsymbol{a}, \boldsymbol{a})$. We will thus show that

$$
\sup _{\boldsymbol{a}, \boldsymbol{b} \in \boldsymbol{A}} X_{n}^{*}(\boldsymbol{a}, \boldsymbol{b})=o_{P}(1), \quad \text { where } \quad X_{n}^{*}(\boldsymbol{a}, \boldsymbol{b})=\sup _{x \in \mathcal{K}}\left|\frac{1}{\sqrt{n}} \sum_{t=1}^{n} z_{t, n}^{*}(x, \boldsymbol{a}, \boldsymbol{b})\right| .
$$

Let $\varepsilon>0$ such that $N:=2 A / \varepsilon$ is an integer and define $a(k)=-A+k \varepsilon$ for $1 \leq k \leq N$. For $1 \leq$ $k_{1}, k_{2}, \ldots k_{d} \leq N$, let $\boldsymbol{k}=\left(k_{1}, \ldots, k_{d}\right)$ and consider the grid of $N^{d}$ points $\boldsymbol{a}(\boldsymbol{k})=\left(a\left(k_{1}\right), \ldots, a\left(k_{d}\right)\right)$. Let also $\boldsymbol{A}(\boldsymbol{k})=\left\{\left(a_{1}, \ldots, a_{d}\right) \in \boldsymbol{A} \mid a\left(k_{j}\right)-\varepsilon \leq a_{j} \leq a\left(k_{j}\right), j \in\{1, \ldots, d\}\right\}$. Define, for $i \geq 1$, and $\boldsymbol{s}_{i}=\left(\boldsymbol{s}_{i}^{(1)}, \boldsymbol{s}_{i}^{(2)}\right)=\left(s_{i 1}^{(1)}, \ldots, s_{i d}^{(1)}, s_{i 1}^{(2)}, \ldots, s_{i d}^{(2)}\right)$

$$
\begin{aligned}
& \overline{\boldsymbol{a}}_{s_{i}^{(\ell)}}(\boldsymbol{k})=\left(\bar{a}_{s_{i 1}^{(\ell)}}\left(k_{1}\right), \ldots, \bar{a}_{s_{i d}^{(\ell)}}\left(k_{d}\right)\right), \quad \bar{a}_{s_{i j}^{(\ell)}}\left(k_{j}\right)=a\left(k_{j}\right) \mathbb{1}_{s_{i j}^{(\ell)}=1}+\left\{a\left(k_{j}\right)-\varepsilon\right\} \mathbb{1}_{s_{i j}^{(\ell)}=-1}, \\
& \underline{\boldsymbol{a}}_{s_{i}^{(\ell)}}(\boldsymbol{k})=\left(\underline{a}_{s_{i 1}^{(\ell)}}\left(k_{1}\right), \ldots, \underline{a}_{s_{i d}^{(\ell)}}\left(k_{d}\right)\right), \quad \underline{a}_{s_{i j}^{(\ell)}}\left(k_{j}\right)=a\left(k_{j}\right) \mathbb{1}_{s_{i j}^{(\ell)}=-1}+\left\{a\left(k_{j}\right)-\varepsilon\right\} \mathbb{1}_{s_{i j}^{(\ell)}=1} .
\end{aligned}
$$

We have, for $j=1, \ldots, d$,

$$
\begin{aligned}
& F\left(x \gamma_{t, n}^{*}(\boldsymbol{a})+\lambda_{t, n}^{*}\left(b_{1}, \ldots, b_{j-1}, a\left(k_{j}\right), b_{j+1}, \ldots, b_{d}\right)\right)-F\left(x \gamma_{t, n}^{*}(\boldsymbol{a})+\lambda_{t, n}^{*}(\boldsymbol{b})\right) \\
= & f\left\{x \gamma_{t, n}^{*}(\boldsymbol{a})+\lambda_{t, n}^{*}\left(\boldsymbol{b}_{t, j}\right)\right\} \frac{a\left(k_{j}\right)-b_{j}}{\sqrt{n}} \frac{1}{\sigma_{t}\left(\boldsymbol{\theta}_{0}\right)} \frac{\partial m_{t}\left(\boldsymbol{\theta}_{0}\right)}{\partial \boldsymbol{\theta}^{\prime}} \boldsymbol{e}_{j},
\end{aligned}
$$

where $\boldsymbol{e}_{j}$ is the $j$-th element of the canonical basis of $\mathbb{R}^{d}$, and $\boldsymbol{b}_{t, j}$ is a point between the arguments of $\lambda_{t, n}$ above. By A11, we have $\sup _{x} f(x)<\infty$. The absolute value of the latter difference is thus bounded, uniformly in $x \in \mathbb{R}, \boldsymbol{a} \in \boldsymbol{A}$ and $\boldsymbol{b} \in \boldsymbol{A}(\boldsymbol{k})$, by $\quad K \frac{\varepsilon}{\sqrt{n}} \frac{1}{\sigma_{t}\left(\boldsymbol{\theta}_{0}\right)}\left|\frac{\partial m_{t}\left(\boldsymbol{\theta}_{0}\right)}{\partial \boldsymbol{\theta}^{\prime}} \boldsymbol{e}_{j}\right|$.

Similarly, for $j=1, \ldots, d$,

$$
\left|F\left(x \gamma_{t, n}^{*}\left(a_{1}, \ldots, a_{j-1}, a\left(k_{j}\right), a_{j+1}, \ldots, a_{d}\right)+\lambda_{t, n}^{*}(\boldsymbol{b})\right)-F\left(x \gamma_{t, n}^{*}(\boldsymbol{a})+\lambda_{t, n}^{*}(\boldsymbol{b})\right)\right|
$$




$$
=\left|x f\left\{x \gamma_{t, n}^{*}\left(\boldsymbol{a}_{t, j}\right)+\lambda_{t, n}^{*}(\boldsymbol{b})\right\} \frac{a\left(k_{j}\right)-a_{j}}{\sqrt{n}} \frac{1}{\sigma_{t}\left(\boldsymbol{\theta}_{0}\right)} \frac{\partial \sigma_{t}\left(\boldsymbol{\theta}_{0}\right)}{\partial \boldsymbol{\theta}^{\prime}} \boldsymbol{e}_{j}\right| \leq K \frac{\varepsilon}{\sqrt{n}} \frac{1}{\sigma_{t}\left(\boldsymbol{\theta}_{0}\right)}\left|\frac{\partial \sigma_{t}\left(\boldsymbol{\theta}_{0}\right)}{\partial \boldsymbol{\theta}^{\prime}} \boldsymbol{e}_{j}\right|,
$$

uniformly in $x \in \mathcal{K}, \boldsymbol{b} \in \boldsymbol{A}$ and $\boldsymbol{a} \in \boldsymbol{A}(\boldsymbol{k})$. Therefore, for $n$ large enough,

$$
\begin{aligned}
& \sup _{\boldsymbol{a} \in \boldsymbol{A}(\boldsymbol{k}), \boldsymbol{b} \in \boldsymbol{A}(\ell)} \sup _{x \in \mathcal{K}} \sum_{t=1}^{n}\left|F\left(x \gamma_{t, n}^{*}(\boldsymbol{a})+\lambda_{t, n}^{*}(\boldsymbol{b})\right)-F\left(x \gamma_{t, n}^{*}(\boldsymbol{a}(\boldsymbol{k}))+\lambda_{t, n}^{*}(\boldsymbol{b}(\boldsymbol{\ell}))\right)\right| \\
\leq & K \frac{\varepsilon}{\sqrt{n}} \sum_{t=1}^{n}\left\|\frac{1}{\sigma_{t}\left(\boldsymbol{\theta}_{0}\right)} \frac{\partial \sigma_{t}\left(\boldsymbol{\theta}_{0}\right)}{\partial \boldsymbol{\theta}}\right\|+\left\|\frac{1}{\sigma_{t}\left(\boldsymbol{\theta}_{0}\right)} \frac{\partial m_{t}\left(\boldsymbol{\theta}_{0}\right)}{\partial \boldsymbol{\theta}}\right\|:=K \frac{\varepsilon}{\sqrt{n}} \sum_{t=1}^{n} Z_{t} .
\end{aligned}
$$

For $\boldsymbol{a} \in \boldsymbol{A}(\boldsymbol{k})$ and $\boldsymbol{b} \in \boldsymbol{A}(\boldsymbol{\ell})$ we have, for $y_{t-1} \in \mathcal{R}_{i}$ and $x \geq 0$,

$$
x \gamma_{t, n}^{*}\left(\underline{\boldsymbol{a}}_{s_{i}^{(2)}}(\boldsymbol{k})\right)+\lambda_{t, n}^{*}\left(\underline{\boldsymbol{a}}_{s_{i}^{(1)}}(\boldsymbol{\ell})\right) \leq x \gamma_{t, n}^{*}(\boldsymbol{a})+\lambda_{t, n}^{*}(\boldsymbol{b}) \leq x \gamma_{t, n}^{*}\left(\overline{\boldsymbol{a}}_{s_{i}^{(2)}}(\boldsymbol{k})\right)+\lambda_{t, n}^{*}\left(\overline{\boldsymbol{a}}_{s_{i}^{(1)}}(\boldsymbol{\ell})\right),
$$

while, for $x \leq 0$,

$$
x \gamma_{t, n}^{*}\left(\overline{\boldsymbol{a}}_{s_{i}^{(2)}}(\boldsymbol{k})\right)+\lambda_{t, n}^{*}\left(\underline{\boldsymbol{a}}_{s_{i}^{(1)}}(\boldsymbol{\ell})\right) \leq x \gamma_{t, n}^{*}(\boldsymbol{a})+\lambda_{t, n}^{*}(\boldsymbol{b}) \leq x \gamma_{t, n}^{*}\left(\underline{\boldsymbol{a}}_{s_{i}^{(2)}}(\boldsymbol{k})\right)+\lambda_{t, n}^{*}\left(\overline{\boldsymbol{a}}_{s_{i}^{(1)}}(\boldsymbol{\ell})\right) .
$$

It follows that,

$$
\begin{aligned}
& \sup _{\boldsymbol{a} \in \boldsymbol{A}(\boldsymbol{k}), \boldsymbol{b} \in \boldsymbol{A}(\boldsymbol{\ell})} \sup _{x \in \mathcal{K}}\left|\frac{1}{\sqrt{n}} \sum_{t=1}^{n} z_{t, n}^{*}(x, \boldsymbol{a}, \boldsymbol{b})-z_{t, n}^{*}(x, \boldsymbol{a}(\boldsymbol{k}), \boldsymbol{a}(\boldsymbol{\ell}))\right| \\
& \leq \sup _{\boldsymbol{a} \in \boldsymbol{A}(\boldsymbol{k}), \boldsymbol{b} \in \boldsymbol{A}(\boldsymbol{\ell})} \sup _{x \in \mathcal{K}} \frac{1}{\sqrt{n}} \sum_{t=1}^{n}\left|\mathbb{1}_{\eta_{t} \leq x \gamma_{t, n}^{*}(\boldsymbol{a}(\boldsymbol{k}))+\lambda_{t, n}^{*}(\boldsymbol{a}(\boldsymbol{\ell}))}-\mathbb{1}_{\eta_{t} \leq x \gamma_{t, n}^{*}(\boldsymbol{a})+\lambda_{t, n}^{*}(\boldsymbol{b})}\right| \\
& +\sup _{\boldsymbol{a} \in \boldsymbol{A}(\boldsymbol{k}), \boldsymbol{b} \in \boldsymbol{A}(\ell)} \sup _{x \in \mathcal{K}} \frac{1}{\sqrt{n}} \sum_{t=1}^{n}\left|F\left(x \gamma_{t, n}^{*}(\boldsymbol{a}(\boldsymbol{k}))+\lambda_{t, n}^{*}(\boldsymbol{a}(\boldsymbol{\ell}))\right)-F\left(x \gamma_{t, n}^{*}(\boldsymbol{a})+\lambda_{t, n}^{*}(\boldsymbol{b})\right)\right| \\
& \leq \sup _{x \in \mathcal{K}} \sum_{i \in I} \frac{1}{\sqrt{n}} \sum_{t=1}^{n}\left\{\mathbb{1}_{\eta_{t} \leq x \gamma_{t, n}^{*}\left(\overline{\boldsymbol{a}}_{s_{i}^{(2)}}(\boldsymbol{k})\right)+\lambda_{t, n}^{*}\left(\overline{\boldsymbol{a}}_{s_{i}^{(1)}}(\ell)\right)}-\mathbb{1}_{\eta_{t} \leq x \gamma_{t, n}^{*}\left(\underline{\boldsymbol{a}}_{s_{i}^{(2)}}(\boldsymbol{k})\right)+\lambda_{t, n}^{*}\left(\underline{\boldsymbol{a}}_{s_{i}^{(1)}}(\ell)\right)}\right\} \mathbb{1}_{y_{t-1} \in \mathcal{R}_{i}} \mathbb{1}_{x \geq 0} \\
& +\sup _{x \in \mathcal{K}} \sum_{i \in I} \frac{1}{\sqrt{n}} \sum_{t=1}^{n}\left\{\mathbb{1}_{\eta_{t} \leq x \gamma_{t, n}^{*}\left(\underline{\boldsymbol{a}}_{s_{i}^{(2)}}(\boldsymbol{k})\right)+\lambda_{t, n}^{*}\left(\overline{\boldsymbol{a}}_{s_{i}^{(1)}}(\boldsymbol{\ell})\right)}-\mathbb{1}_{\eta_{t} \leq x \gamma_{t, n}^{*}\left(\overline{\boldsymbol{a}}_{s_{i}^{(2)}}(\boldsymbol{k})\right)+\lambda_{t, n}^{*}\left(\underline{\boldsymbol{a}}_{s_{i}^{(1)}}(\boldsymbol{\ell})\right)}\right\} \mathbb{1}_{y_{\underline{t-1}} \in \mathcal{R}_{i}} \mathbb{1}_{x<0} \\
& +K \frac{\varepsilon}{n} \sum_{t=1}^{n} Z_{t} \\
& =\sup _{x \in \mathcal{K}} \sum_{i \in I} \frac{1}{\sqrt{n}} \sum_{t=1}^{n}\left\{z^{*}\left(x, \overline{\boldsymbol{a}}_{s_{i}^{(2)}}(\boldsymbol{k}), \overline{\boldsymbol{a}}_{s_{i}^{(1)}}(\boldsymbol{\ell})\right)-z^{*}\left(x, \underline{\boldsymbol{a}}_{s_{i}^{(2)}}(\boldsymbol{k}), \underline{\boldsymbol{a}}_{s_{i}^{(1)}}(\boldsymbol{\ell})\right)\right\} \mathbf{1}_{\underline{y_{--1}} \in \mathcal{R}_{i}} \mathbb{1}_{x \geq 0} \\
& +\sup _{x \in \mathcal{K}} \sum_{i \in I} \frac{1}{\sqrt{n}} \sum_{t=1}^{n}\left\{F\left(x \gamma_{t, n}^{*}\left(\overline{\boldsymbol{a}}_{s_{i}^{(2)}}(\boldsymbol{k})\right)+\lambda_{t, n}^{*}\left(\overline{\boldsymbol{a}}_{s_{i}^{(1)}}(\boldsymbol{\ell})\right)\right)-F\left(x \gamma_{t, n}^{*}\left(\underline{\boldsymbol{a}}_{s_{i}^{(2)}}(\boldsymbol{k})\right)+\lambda_{t, n}^{*}\left(\underline{\boldsymbol{a}}_{s_{i}^{(1)}}(\boldsymbol{\ell})\right)\right)\right\} \mathbb{1}_{y_{\underline{t-1}} \in \mathcal{R}_{i}} \mathbb{1}_{x \geq 0} \\
& +\sup _{x \in \mathcal{K}} \sum_{i \in I} \frac{1}{\sqrt{n}} \sum_{t=1}^{n}\left\{z^{*}\left(x, \underline{\boldsymbol{a}}_{s_{i}^{(2)}}(\boldsymbol{k}), \overline{\boldsymbol{a}}_{s_{i}^{(1)}}(\boldsymbol{\ell})\right)-z^{*}\left(x, \overline{\boldsymbol{a}}_{s_{i}^{(2)}}(\boldsymbol{k}), \underline{\boldsymbol{a}}_{s_{i}^{(1)}}(\boldsymbol{\ell})\right)\right\} \mathbb{1}_{y_{\underline{t-1}} \in \mathcal{R}_{i}} \mathbb{1}_{x<0} \\
& +\sup _{x \in \mathcal{K}} \sum_{i \in I} \frac{1}{\sqrt{n}} \sum_{t=1}^{n}\left\{F\left(x \gamma_{t, n}^{*}\left(\underline{\boldsymbol{a}}_{s_{i}^{(2)}}(\boldsymbol{k})\right)+\lambda_{t, n}^{*}\left(\overline{\boldsymbol{a}}_{s_{i}^{(1)}}(\boldsymbol{\ell})\right)\right)-F\left(x \gamma_{t, n}^{*}\left(\overline{\boldsymbol{a}}_{s_{i}^{(2)}}(\boldsymbol{k})\right)+\lambda_{t, n}^{*}\left(\underline{\boldsymbol{a}}_{s_{i}^{(1)}}(\boldsymbol{\ell})\right)\right)\right\} \mathbb{1}_{y_{\underline{t-1}} \in \mathcal{R}_{i}} \mathbb{1}_{x<0} \\
& +K \frac{\varepsilon}{n} \sum_{t=1}^{n} Z_{t}
\end{aligned}
$$




$$
\begin{aligned}
\leq & \boldsymbol{\mathcal { X }}_{n}\left(\left(\overline{\boldsymbol{a}}_{s_{i}^{(2)}}(\boldsymbol{k})\right),\left(\overline{\boldsymbol{a}}_{s_{i}^{(1)}}(\boldsymbol{\ell})\right)\right)+\boldsymbol{\mathcal { X }}_{n}\left(\left(\underline{\boldsymbol{a}}_{s_{i}^{(2)}}(\boldsymbol{k})\right),\left(\underline{\boldsymbol{a}}_{s_{i}^{(1)}}(\boldsymbol{\ell})\right)\right) \\
& +\boldsymbol{\mathcal { X }}_{n}\left(\left(\underline{\boldsymbol{a}}_{s_{i}^{(2)}}(\boldsymbol{k})\right),\left(\overline{\boldsymbol{a}}_{s_{i}^{(1)}}(\boldsymbol{\ell})\right)\right)+\boldsymbol{\mathcal { X }}_{n}\left(\left(\overline{\boldsymbol{a}}_{s_{i}^{(2)}}(\boldsymbol{k})\right),\left(\underline{\boldsymbol{a}}_{s_{i}^{(1)}}(\boldsymbol{\ell})\right)\right)+3 K \frac{\varepsilon}{n} \sum_{t=1}^{n} Z_{t} .
\end{aligned}
$$

Note that

$$
\begin{aligned}
\sup _{\boldsymbol{a}, \boldsymbol{b} \in \boldsymbol{A}} X_{n}^{*}(\boldsymbol{a}, \boldsymbol{b}) \leq & \max _{\boldsymbol{k}, \ell \in\{1, \ldots, N\}^{d}} \sup _{\boldsymbol{a} \in \boldsymbol{A}(\boldsymbol{k}), \boldsymbol{b} \in \boldsymbol{B}(\ell)} \sup _{x \in \mathcal{K}}\left|\frac{1}{\sqrt{n}} \sum_{t=1}^{n}\left[z_{t, n}^{*}(x, \boldsymbol{a}, \boldsymbol{b})-z_{t, n}^{*}(x, \boldsymbol{a}(\boldsymbol{k}), \boldsymbol{b}(\boldsymbol{\ell}))\right]\right| \\
& +\max _{\boldsymbol{k}, \boldsymbol{\ell} \in\{1, \ldots, N\}^{d}} X_{n}^{*}(\boldsymbol{a}(\boldsymbol{k}), \boldsymbol{a}(\boldsymbol{\ell})) \\
\leq & \frac{3 K \varepsilon}{n} \sum_{t=1}^{n} Z_{t}+5 \max _{\left(\boldsymbol{a}_{i}\right),\left(\boldsymbol{b}_{i}\right)} \boldsymbol{\mathcal { X }}_{n}\left(\left(\boldsymbol{a}_{i}\right),\left(\boldsymbol{b}_{i}\right)\right),
\end{aligned}
$$

where the last max is taken over all sequences of the form $\overline{\boldsymbol{a}}_{s_{i}^{(\ell)}}(\boldsymbol{k})$ or $\underline{\boldsymbol{a}}_{s_{i}^{(\ell)}}(\boldsymbol{k}), \ell \in\{1,2\}$ and $\boldsymbol{k}, \boldsymbol{\ell} \in\{1, \ldots, N\}^{d}$. The end of the proof of (33) is similar to that of (21) using Lemma 11.

Proof that $\sup _{x \in \mathbb{R}}\left|\widehat{e}_{n, 7}(x)\right|=o_{P}(1)$ in Lemma 14 .

By B2-B3, $\left|\eta_{t}\left(\widehat{\boldsymbol{\theta}}_{n}\right)-\tilde{\eta}_{t}\left(\widehat{\boldsymbol{\theta}}_{n}\right)\right| \leq K_{t} \rho^{t}$. It follows that

$$
\begin{aligned}
\left|\mathbb{1}_{\eta_{t} \leq x \tilde{\chi}_{t, n}+\tilde{\lambda}_{t, n}}-\mathbb{1}_{\eta_{t} \leq x \chi_{t, n}+\lambda_{t, n}}\right| & =\left|\mathbb{1}_{\tilde{\eta}_{t}\left(\widehat{\boldsymbol{\theta}}_{n}\right) \leq x}-\mathbb{1}_{\eta_{t}\left(\widehat{\boldsymbol{\theta}}_{n}\right) \leq x}\right| \leq \mathbb{1}_{x-K_{t} \rho^{t} \leq \eta_{t}\left(\widehat{\boldsymbol{\theta}}_{n}\right) \leq x+K_{t} \rho^{t}} \\
& \leq \mathbb{1}_{x-\rho^{t / 2} \leq \eta_{t}\left(\widehat{\boldsymbol{\theta}}_{n}\right) \leq x+\rho^{t / 2}}+\mathbb{1}_{K_{t} \rho^{t / 2} \geq 1} .
\end{aligned}
$$

We have, by B3 and Markov inequality

$$
E \frac{1}{\sqrt{n}} \sum_{t=1}^{n} \mathbb{1}_{K_{t} \rho^{t / 2} \geq 1}=\frac{1}{\sqrt{n}} \sum_{t=1}^{n} P\left(K_{t} \rho^{t / 2} \geq 1\right) \leq \frac{1}{\sqrt{n}} \sum_{t=1}^{n} E\left(K_{t}^{r} \rho^{t r / 2}\right)=O\left(n^{-1 / 2}\right) .
$$

Letting

$$
d_{t, n}(x)=\mathbb{1}_{x-\rho^{t / 2} \leq \eta_{t}\left(\widehat{\boldsymbol{\theta}}_{n}\right) \leq x+\rho^{t / 2}}=\mathbb{1}_{\chi_{t, n} x-\chi_{t, n} \rho^{t / 2}+\lambda_{t, n} \leq \eta_{t} \leq \chi_{t, n} x+\chi_{t, n} \rho^{t / 2}+\lambda_{t, n}}
$$

we have

$$
\sup _{x \in \mathbb{R}} \frac{1}{\sqrt{n}} \sum_{t=1}^{n} d_{t, n}(x) \leq \sum_{i=1}^{5} c_{i, n}
$$

with

$$
\begin{aligned}
c_{1, n} & =\sup _{x \in \mathbb{R}} \frac{1}{\sqrt{n}} \sum_{t=1}^{\left\lceil n^{1 / 4}\right\rceil-1} d_{t, n}(x) \leq \frac{1}{n^{1 / 4}}, \\
c_{2, n} & =\sup _{x \in \mathbb{R}} \frac{1}{\sqrt{n}} \sum_{t=\left\lceil n^{1 / 4}\right\rceil}^{n} \mathbb{1}_{\chi_{t, n} x-b_{n} \rho_{n}+\lambda_{t, n} \leq \eta_{t} \leq \chi_{t, n} x+b_{n} \rho_{n}+\lambda_{t, n}} \mathbb{1}_{A_{n}}, \quad \rho_{n}=\rho^{\frac{n^{1 / 4}}{2}}, \\
c_{3, n} & =\frac{1}{\sqrt{n}} \sum_{t=1}^{n} \mathbb{1}_{\chi_{t, n} \geq b_{n}} \mathbb{1}_{\sqrt{n}\left\|\widehat{\boldsymbol{\theta}}_{n}-\boldsymbol{\theta}_{0}\right\| \leq n^{s}}, \quad c_{4, n}=\sqrt{n} \mathbb{1}_{\sqrt{n}\left\|\widehat{\boldsymbol{\theta}}_{n}-\boldsymbol{\theta}_{0}\right\| \geq n^{s}}, \quad c_{5, n}=\sqrt{n} \mathbb{1}_{A_{n}^{c}},
\end{aligned}
$$

where $\left(a_{n}\right)$ and $\left(b_{n}\right)$ are sequences of real numbers, $A_{n}=\left\{\min _{1 \leq t<t^{\prime} \leq n}\left|\eta_{t}-\eta_{t^{\prime}}\right| \geq a_{n}\right\}$ and $s>0$. Note that $c_{2, n}=1 / \sqrt{n}$ when $a_{n} \geq 2 b_{n} \rho_{n}$. Now, for some $\boldsymbol{\theta}_{t}$ between $\widehat{\boldsymbol{\theta}}_{n}$ and $\boldsymbol{\theta}_{0}$, we have

$$
\chi_{t, n}=1+\frac{1}{\sigma_{t}\left(\boldsymbol{\theta}_{0}\right)} \frac{\partial \sigma_{t}\left(\boldsymbol{\theta}_{t}\right)}{\partial \boldsymbol{\theta}^{\prime}}\left(\widehat{\boldsymbol{\theta}}_{n}-\boldsymbol{\theta}_{0}\right) .
$$


Using B7 and choosing for instance $b_{n}=\sqrt{n}+1$, we obtain

$$
\begin{aligned}
E c_{3, n} & =\frac{1}{\sqrt{n}} \sum_{t=1}^{n} P\left(\chi_{t, n} \geq b_{n}, \sqrt{n}\left\|\widehat{\boldsymbol{\theta}}_{n}-\boldsymbol{\theta}_{0}\right\| \leq n^{s}\right) \\
& \leq \frac{1}{\sqrt{n}} \sum_{t=1}^{n} P\left(\sup _{\boldsymbol{\theta} \in V\left(\boldsymbol{\theta}_{0}\right)}\left\|\frac{1}{\sigma_{t}\left(\boldsymbol{\theta}_{0}\right)} \frac{\partial \sigma_{t}(\boldsymbol{\theta})}{\partial \boldsymbol{\theta}}\right\| n^{s-1 / 2} \geq \sqrt{n}\right)=o(1)
\end{aligned}
$$

By Lemma 1 and B5, we have almost surely $c_{4, n}=0$ for $n$ large enough. In the proof of Lemma 7 , we have seen that $E c_{5, n}=o(1)$ when, for instance, $a_{n}=n^{-3}$. Hence the lemma is established.

Detailed proof of Lemma 14. From (34) and a similar expansion for $\lambda_{t, n}$

$$
\begin{aligned}
& \left|x \chi_{t, n}+\lambda_{t, n}-\left(x \chi_{t, n}^{*}+\lambda_{t, n}^{*}\right)\right| \\
= & \left|\frac{1}{\sigma_{t}\left(\boldsymbol{\theta}_{0}\right)}\left\{x\left(\frac{\partial \sigma_{t}\left(\boldsymbol{\theta}_{t}\right)}{\partial \boldsymbol{\theta}^{\prime}}-\frac{\partial \sigma_{t}\left(\boldsymbol{\theta}_{0}\right)}{\partial \boldsymbol{\theta}^{\prime}}\right)+\left(\frac{\partial m_{t}\left(\boldsymbol{\theta}_{t}\right)}{\partial \boldsymbol{\theta}^{\prime}}-\frac{\partial m_{t}\left(\boldsymbol{\theta}_{0}\right)}{\partial \boldsymbol{\theta}^{\prime}}\right)\right\}\left(\widehat{\boldsymbol{\theta}}_{n}-\boldsymbol{\theta}_{0}\right)\right| \\
\leq & \frac{1}{\sigma_{t}\left(\boldsymbol{\theta}_{0}\right)}\left\{|x|\left\|\frac{\partial \sigma_{t}\left(\boldsymbol{\theta}_{t}\right)}{\partial \boldsymbol{\theta}}-\frac{\partial \sigma_{t}\left(\boldsymbol{\theta}_{0}\right)}{\partial \boldsymbol{\theta}}\right\|+\left\|\frac{\partial m_{t}\left(\boldsymbol{\theta}_{t}\right)}{\partial \boldsymbol{\theta}}-\frac{\partial m_{t}\left(\boldsymbol{\theta}_{0}\right)}{\partial \boldsymbol{\theta}}\right\|\right\}\left\|\widehat{\boldsymbol{\theta}}_{n}-\boldsymbol{\theta}_{0}\right\| \\
\leq & \frac{1}{\sigma_{t}\left(\boldsymbol{\theta}_{0}\right)}\left\{|x|\left\|\frac{\partial^{2} \sigma_{t}\left(\overline{\boldsymbol{\theta}}_{t}\right)}{\partial \boldsymbol{\theta} \partial \boldsymbol{\theta}^{\prime}}\left(\boldsymbol{\theta}_{t}-\boldsymbol{\theta}_{0}\right)\right\|+\left\|\frac{\partial^{2} m_{t}\left(\underline{\boldsymbol{\theta}}_{t}\right)}{\partial \boldsymbol{\theta} \partial \boldsymbol{\theta}^{\prime}}\left(\boldsymbol{\theta}_{t}-\boldsymbol{\theta}_{0}\right)\right\|\right\}\left\|\widehat{\boldsymbol{\theta}}_{n}-\boldsymbol{\theta}_{0}\right\| \\
\leq & \frac{2}{\sigma_{t}\left(\boldsymbol{\theta}_{0}\right)} \sup _{\boldsymbol{\theta} \in V\left(\boldsymbol{\theta}_{0}\right)}\left\{|x|\left\|\frac{\partial^{2} \sigma_{t}(\boldsymbol{\theta})}{\partial \boldsymbol{\theta} \partial \boldsymbol{\theta}^{\prime}}\right\|+\left\|\frac{\partial^{2} m_{t}(\boldsymbol{\theta})}{\partial \boldsymbol{\theta} \partial \boldsymbol{\theta}^{\prime}}\right\|\right\}\left\|\widehat{\boldsymbol{\theta}}_{n}-\boldsymbol{\theta}_{0}\right\|^{2}:=\delta_{t}(x)\left\|\widehat{\boldsymbol{\theta}}_{n}-\boldsymbol{\theta}_{0}\right\|^{2},
\end{aligned}
$$

where $\boldsymbol{\theta}_{t}, \underline{\boldsymbol{\theta}}_{t}$ and $\overline{\boldsymbol{\theta}}_{t}$ are between $\boldsymbol{\theta}_{0}$ and $\widehat{\boldsymbol{\theta}}_{n}$. It follows that, for any $\Delta_{n}>0$,

$$
\begin{aligned}
\left|\widehat{e}_{n, 8}\left(x_{n}\right)\right| & \leq \frac{1}{\sqrt{n}} \sum_{t=1}^{n}\left|\mathbb{1}_{\eta_{t} \leq x_{n} \chi_{t, n}+\lambda_{t, n}}-\mathbb{1}_{\eta_{t} \leq x_{n} \chi_{t, n}^{*}+\lambda_{t, n}^{*}}\right| \\
& \leq \frac{1}{\sqrt{n}} \sum_{t=1}^{n} \mathbb{1}_{x_{n} \chi_{t, n}^{*}+\lambda_{t, n}^{*}-\delta_{t}\left(x_{n}\right) \Delta_{n}^{2} \leq \eta_{t} \leq x_{n} \chi_{t, n}^{*}+\lambda_{t, n}^{*}+\delta_{t}\left(x_{n}\right) \Delta_{n}^{2}}+\sqrt{n} \mathbb{1}_{\left\|\widehat{\boldsymbol{\theta}}_{n}-\boldsymbol{\theta}_{0}\right\|>\Delta_{n}} .
\end{aligned}
$$

Let $\Delta_{n}=n^{s-1 / 2}$, for some $s>0$. The last term in (35) is equal to $c_{4, n}=0$ for sufficiently large $n$. Now, first suppose that the sequence $\left(x_{n}\right)$ is non random, $x_{n} \rightarrow x$. Note that the sequence $\left(\delta_{t}\left(x_{n}\right)\right)_{t}$ is stationary, and integrable by Assumptions A9 and B7. Let $f_{t, n}$ the conditional density of $\eta_{t}$ introduced in Assumption B8, and let $K$ its uniform upper bound. Then, by the law of iterated expectations, the expectation of the first term in (35) is bounded, for $n>n_{0}$, by

$$
2 \sqrt{n} \Delta_{n}^{2} K E\left\{\delta_{1}\left(x_{n}\right)\right\}+\frac{t(n)}{\sqrt{n}}=o(1)
$$

The conclusion follows in case i). Turning to case ii), using Lemma 4.4 in Kreiss (1987), we can deduce that the conclusion also holds. 


\section{QMLE of the location scale model}

\section{D.1. Consequence of Assumption A12* for the QMLE}

Consider the function $g: c \mapsto g(c)=Q_{n}\left(\widehat{\boldsymbol{\theta}}_{n, c}\right)=\frac{1}{n} \sum_{t=1}^{n} \frac{\left\{y_{t}-\tilde{m}_{t}\left(\widehat{\boldsymbol{\theta}}_{n}\right)\right\}^{2}}{c^{2} \tilde{\sigma}_{t}^{2}\left(\widehat{\boldsymbol{\theta}}_{n}\right)}+\log \left\{c^{2} \tilde{\sigma}_{t}^{2}\left(\widehat{\boldsymbol{\theta}}_{n}\right)\right\}$. Because the function $g$ reaches its minimum for $c=1$, we must have $\widehat{\mu}_{2}=1$ a.s.

Now we have

$$
\widehat{\mu}_{2}:=\frac{1}{n} \sum_{t=1}^{n} \tilde{\eta}_{t}^{2}\left(\widehat{\boldsymbol{\theta}}_{n}\right)=\frac{1}{n} \sum_{t=1}^{n} \eta_{t}^{2}\left(\widehat{\boldsymbol{\theta}}_{n}\right)+o_{P}(1)=\frac{1}{n} \sum_{t=1}^{n} \eta_{t}^{2}+\frac{\partial \mu_{2, n}\left(\boldsymbol{\theta}_{0}\right)}{\partial \boldsymbol{\theta}^{\prime}}\left(\widehat{\boldsymbol{\theta}}_{n}-\boldsymbol{\theta}_{0}\right)+o_{P}(1),
$$

where

$$
\mu_{2, n}(\boldsymbol{\theta})=\frac{1}{n} \sum_{t=1}^{n} \eta_{t}^{2}(\boldsymbol{\theta}), \quad \frac{\partial \mu_{2, n}\left(\boldsymbol{\theta}_{0}\right)}{\partial \boldsymbol{\theta}^{\prime}}=\frac{-2}{n} \sum_{t=1}^{n} \eta_{t}\left\{\frac{1}{\sigma_{t}} \frac{\partial m_{t}\left(\boldsymbol{\theta}_{0}\right)}{\partial \boldsymbol{\theta}^{\prime}}+\eta_{t} \frac{1}{\sigma_{t}} \frac{\partial \sigma_{t}\left(\boldsymbol{\theta}_{0}\right)}{\partial \boldsymbol{\theta}^{\prime}}\right\}=-2 \boldsymbol{\Omega}_{\sigma}^{\prime}+o_{P}(1) .
$$

Thus

$$
\sqrt{n}\left(\widehat{\mu}_{2}-1\right)=\frac{1}{\sqrt{n}} \sum_{t=1}^{n}\left(\eta_{t}^{2}-1\right)-2 \boldsymbol{\Omega}_{\sigma}^{\prime} \sqrt{n}\left(\widehat{\boldsymbol{\theta}}_{n}-\boldsymbol{\theta}_{0}\right)+o_{P}(1) .
$$

It follows from (36) and (5) that

$$
\begin{aligned}
\sqrt{n}\left(\widehat{\mu}_{2}-1\right)= & -4 \boldsymbol{\Omega}_{\sigma}^{\prime} \boldsymbol{J}^{-1} \frac{1}{\sqrt{n}} \sum_{t=1}^{n} \eta_{t} \frac{1}{\sigma_{t}} \frac{\partial m_{t}\left(\boldsymbol{\theta}_{0}\right)}{\partial \boldsymbol{\theta}} \\
& +\frac{1}{\sqrt{n}} \sum_{t=1}^{n}\left(\eta_{t}^{2}-1\right)\left(1-4 \boldsymbol{\Omega}_{\sigma}^{\prime} \boldsymbol{J}^{-1} \frac{1}{\sigma_{t}} \frac{\partial \sigma_{t}\left(\boldsymbol{\theta}_{0}\right)}{\partial \boldsymbol{\theta}}\right)+o_{P}(1) .
\end{aligned}
$$

Since, in (37), the asymptotic distribution of the l.h.s. is degenerate, we deduce that

$$
\begin{aligned}
0= & 16 \boldsymbol{\Omega}_{\sigma}^{\prime} \boldsymbol{J}^{-1}\left\{\boldsymbol{J}_{m}+\left(\kappa_{4}-1\right) \boldsymbol{J}_{\sigma}+\mu_{3}\left(\boldsymbol{J}_{m \sigma}+\boldsymbol{J}_{\sigma m}\right)\right\} \boldsymbol{J}^{-1} \boldsymbol{\Omega}_{\sigma}+\left(\kappa_{4}-1\right)\left(1-8 \boldsymbol{\Omega}_{\sigma}^{\prime} \boldsymbol{J}^{-1} \boldsymbol{\Omega}_{\sigma}\right) \\
& -4 \mu_{3}\left(\boldsymbol{\Omega}_{\sigma}^{\prime} \boldsymbol{J}^{-1} \boldsymbol{\Omega}_{m}+\boldsymbol{\Omega}_{m}^{\prime} \boldsymbol{J}^{-1} \boldsymbol{\Omega}_{\sigma}\right) .
\end{aligned}
$$

We retrieve the fact that in the absence of location $m, \boldsymbol{J}_{\sigma}=\boldsymbol{J} / 4$ and thus $\boldsymbol{\Omega}_{\sigma}^{\prime} \boldsymbol{J}_{\sigma}^{-1} \boldsymbol{\Omega}_{\sigma}=1$. In the Gaussian case we also get $\boldsymbol{\Omega}_{\sigma}^{\prime} \boldsymbol{J}_{\sigma}^{-1} \boldsymbol{\Omega}_{\sigma}=1$ (with or without a location).

Finally, (38) writes

$$
4 \boldsymbol{\Omega}_{\sigma}^{\prime} \boldsymbol{\Sigma} \boldsymbol{\Omega}_{\sigma}+\left(\kappa_{4}-1\right)\left(1-8 \boldsymbol{\Omega}_{\sigma}^{\prime} \boldsymbol{J}^{-1} \boldsymbol{\Omega}_{\sigma}\right)-4 \mu_{3}\left(\boldsymbol{\Omega}_{\sigma}^{\prime} \boldsymbol{J}^{-1} \boldsymbol{\Omega}_{m}+\boldsymbol{\Omega}_{m}^{\prime} \boldsymbol{J}^{-1} \boldsymbol{\Omega}_{\sigma}\right)=0 .
$$

\section{D.2. Proof of Corollary 1}

Let $\boldsymbol{\theta}=\left(\boldsymbol{\varphi}^{\prime}, \boldsymbol{\vartheta}^{\prime}\right)^{\prime}$. We have

$$
\begin{gathered}
\boldsymbol{\Omega}_{m}=\left(E\left(\frac{1}{\sigma_{t}} \frac{\partial m_{t}\left(\boldsymbol{\varphi}_{0}\right)}{\partial \boldsymbol{\varphi}^{\prime}}\right), \mathbf{0}\right)^{\prime}, \quad \boldsymbol{\Omega}_{\sigma}=\left(\mathbf{0}, E\left(\frac{1}{\sigma_{t}} \frac{\partial \sigma_{t}\left(\boldsymbol{\vartheta}_{0}\right)}{\partial \boldsymbol{\vartheta}^{\prime}}\right)\right)^{\prime}, \quad \boldsymbol{J}_{m \sigma}=\boldsymbol{J}_{\sigma m}^{\prime}=\mathbf{0}, \\
\boldsymbol{J}_{m}=\left(\begin{array}{cc}
\boldsymbol{K} & \mathbf{0} \\
\mathbf{0} & 0
\end{array}\right), \quad \boldsymbol{J}_{\sigma}=\left(\begin{array}{cc}
\mathbf{0} & \mathbf{0} \\
\mathbf{0} & \boldsymbol{L}
\end{array}\right), \quad \boldsymbol{J}=\left(\begin{array}{cc}
2 \boldsymbol{K} & \mathbf{0} \\
\mathbf{0} & 4 \boldsymbol{L}
\end{array}\right), \quad \boldsymbol{\Sigma}=\left(\begin{array}{cc}
\boldsymbol{K}^{-1} & \mathbf{0} \\
\mathbf{0} & \frac{\kappa_{4}-1}{4} \boldsymbol{L}^{-1}
\end{array}\right),
\end{gathered}
$$


where $\boldsymbol{K}=E\left(\frac{1}{\sigma_{t}^{2}} \frac{\partial m_{t}\left(\boldsymbol{\varphi}_{0}\right)}{\partial \varphi} \frac{\partial m_{t}\left(\boldsymbol{\varphi}_{0}\right)}{\partial \boldsymbol{\varphi}^{\prime}}\right), \boldsymbol{L}=E\left(\frac{1}{\sigma_{t}^{2}} \frac{\partial \sigma_{t}\left(\boldsymbol{\vartheta}_{0}\right)}{\partial \vartheta} \frac{\partial \sigma_{t}\left(\boldsymbol{\vartheta}_{0}\right)}{\partial \boldsymbol{\vartheta}^{\prime}}\right)$. Moreover, (38) entails

$$
E\left(\frac{1}{\sigma_{t}} \frac{\partial \sigma_{t}\left(\boldsymbol{\vartheta}_{0}\right)}{\partial \boldsymbol{\vartheta}^{\prime}}\right)\left\{E\left(\frac{1}{\sigma_{t}^{2}} \frac{\partial \sigma_{t}\left(\boldsymbol{\vartheta}_{0}\right)}{\partial \boldsymbol{\vartheta}} \frac{\partial \sigma_{t}\left(\boldsymbol{\vartheta}_{0}\right)}{\partial \boldsymbol{\vartheta}^{\prime}}\right)\right\}^{-1} E\left(\frac{1}{\sigma_{t}} \frac{\partial \sigma_{t}\left(\boldsymbol{\vartheta}_{0}\right)}{\partial \boldsymbol{\vartheta}}\right)=1
$$

The conclusion follows.

\section{D.3. Proof of Remark 5}

We have

$\boldsymbol{\Omega}_{m}=\left(\begin{array}{c}E\left(\frac{X_{t-1}}{\sigma_{t}}\right) \\ 0\end{array}\right), \quad \boldsymbol{\Omega}_{\sigma}=\left(\begin{array}{c}0 \\ E\left(\frac{X_{t-1}^{2}}{2 \sigma_{t}^{2}}\right)\end{array}\right), \quad \boldsymbol{J}_{m}=\left(\begin{array}{cc}E\left(\frac{X_{t-1}^{2}}{\sigma_{t}^{2}}\right) & 0 \\ 0 & 0\end{array}\right), \quad \boldsymbol{J}_{\sigma}=\left(\begin{array}{cc}0 & 0 \\ 0 & \frac{1}{4} E\left(\frac{X_{t-1}^{4}}{\sigma_{t}^{4}}\right)\end{array}\right)$.

Hence

$$
\begin{aligned}
& \boldsymbol{J}=\left(\begin{array}{cc}
2 E\left(\frac{X_{t-1}^{2}}{\sigma_{t}^{2}}\right) & 0 \\
0 & E\left(\frac{X_{t-1}^{4}}{\sigma_{t}^{4}}\right)
\end{array}\right), \quad \boldsymbol{\Sigma}=\left(\begin{array}{cc}
\left\{E\left(\frac{X_{t-1}^{2}}{\sigma_{t}^{2}}\right)\right\}^{-1} & 0 \\
0 & \left(\kappa_{4}-1\right)\left\{E\left(\frac{X_{t-1}^{4}}{\sigma_{t}^{4}}\right)\right\}^{-1}
\end{array}\right), \\
& \boldsymbol{H}(x)=f(x)\left(\begin{array}{c}
E\left(\frac{X_{t-1}}{\sigma_{t}}\right) \\
x E\left(\frac{X_{t-1}^{2}}{2 \sigma_{t}^{2}}\right)
\end{array}\right), \quad \boldsymbol{\Lambda}=\left(\begin{array}{cc}
\frac{E\left(\frac{x_{t-1}}{\sigma_{t}}\right)}{E\left(\frac{X_{t-1}^{2}}{\sigma_{t}^{2}}\right)} & 0 \\
0 & \frac{E\left(\frac{X_{t-1}^{2}}{\sigma_{t}^{2}}\right)}{E\left(\frac{X_{t-1}^{4}}{\sigma_{t}^{4}}\right)}
\end{array}\right), \\
& \boldsymbol{H}(x)^{\prime} \boldsymbol{\Sigma} \boldsymbol{H}(x)=f(x)^{2} \frac{\left\{E\left(\frac{X_{t-1}}{\sigma_{t}}\right)\right\}^{2}}{E\left(\frac{X_{t-1}^{2}}{\sigma_{t}^{2}}\right)}+\{x f(x)\}^{2} \frac{\kappa_{4}-1}{4} \frac{\left\{E\left(\frac{X_{t-1}^{2}}{\sigma_{t}^{2}}\right)\right\}^{2}}{E\left(\frac{X_{t-1}^{4}}{\sigma_{t}^{4}}\right)}, \\
& 2 \boldsymbol{H}(x)^{\prime} \boldsymbol{\Lambda} \varrho(x)=2 f(x) \frac{\left\{E\left(\frac{X_{t-1}}{\sigma_{t}}\right)\right\}^{2}}{E\left(\frac{X_{t-1}^{2}}{\sigma_{t}^{2}}\right)} E\left\{\mathbb{1}_{\eta_{0}<x} \eta_{0}\right\}+x f(x) \frac{\left\{E\left(\frac{X_{t-1}^{2}}{\sigma_{t}^{2}}\right)\right\}^{2}}{E\left(\frac{X_{t-1}^{4}}{\sigma_{t}^{4}}\right)} E\left\{\mathbb{1}_{\eta_{0}<x}\left(\eta_{0}^{2}-1\right)\right\} .
\end{aligned}
$$

Finally, for this model,

$$
\begin{aligned}
\operatorname{Var}_{a s}\left\{\sqrt{n}\left(\widehat{F}_{n}\left(x_{n}\right)-F\left(x_{n}\right)\right)\right\}= & F(x)\{1-F(x)\}+f(x) \frac{\left\{E\left(\frac{X_{t-1}}{\sigma_{t}}\right)\right\}^{2}}{E\left(\frac{X_{t-1}^{2}}{\sigma_{t}^{2}}\right)}\left\{f(x)+2 E\left\{\mathbb{1}_{\eta_{0}<x} \eta_{0}\right\}\right\} \\
& +x f(x) \frac{\left\{E\left(\frac{X_{t-1}^{2}}{\sigma_{t}^{2}}\right)\right\}^{2}}{E\left(\frac{X_{t-1}^{4}}{\sigma_{t}^{4}}\right)}\left\{x f(x) \frac{\kappa_{4}-1}{4}+E\left\{\mathbb{1}_{\eta_{0}<x}\left(\eta_{0}^{2}-1\right)\right\}\right\} .
\end{aligned}
$$

which is clearly model-dependent. Note that if the distribution of $\eta_{t}$ is symmetric we have $E\left(\frac{X_{t-1}}{\sigma_{t}}\right)=0$ and thus the second term in the right-hand side vanishes. If, in addition, $\alpha_{0} \rightarrow 0$, the asymptotic variance converges to

$$
F(x)\{1-F(x)\}+x f(x) \frac{1+\phi_{0}^{2}}{6 \phi_{0}^{2}}\left\{x f(x) \frac{\kappa_{4}-1}{4}+E\left\{\mathbb{1}_{\eta_{0}<x}\left(\eta_{0}^{2}-1\right)\right\}\right\} .
$$




\section{D.4. Proof of Corollary 2}

Let $\boldsymbol{\theta}=\left(\boldsymbol{\varphi}^{\prime}, \boldsymbol{\vartheta}^{\prime}\right)^{\prime}$, where $\boldsymbol{\varphi}=\left(\phi_{1}, \ldots, \phi_{P}, \psi_{1}, \ldots, \psi_{Q}\right)^{\prime}, \boldsymbol{\vartheta}=\left(\omega, \alpha_{1}, \ldots, \alpha_{q}, \beta_{1}, \ldots, \beta_{p}\right)^{\prime}$, and $m_{t}=$ $m_{t}(\boldsymbol{\varphi}), \sigma_{t}=\sigma_{t}(\boldsymbol{\varphi}, \boldsymbol{\vartheta})$. We know that the asymptotic variance $\boldsymbol{\Sigma}$ of the QMLE is block-diagonal (see Francq and Zakoian (2019), Remark 7.4). Moreover, we have $\frac{\partial m_{t}\left(\boldsymbol{\varphi}_{0}\right)}{\partial \phi_{i}}=\Theta(L)^{-1} X_{t-i}$ and $\frac{\partial m_{t}\left(\boldsymbol{\varphi}_{0}\right)}{\partial \psi_{j}}=-\Theta(L)^{-1} \epsilon_{t-j}$. Hence $E\left(\frac{1}{\sigma_{t}} \frac{\partial m_{t}\left(\boldsymbol{\varphi}_{0}\right)}{\partial \boldsymbol{\varphi}}\right)=0$ using the fact that $\sigma_{t}$ is an even function of the $\eta_{t-i}, i>0$. Since $\frac{\partial m_{t}\left(\boldsymbol{\varphi}_{0}\right)}{\partial \boldsymbol{\vartheta}}=0$ we thus have $\boldsymbol{\Omega}_{m}=\mathbf{0}$ and it can similarly be shown that $\boldsymbol{\Omega}_{\sigma}$ is of the form $\boldsymbol{\Omega}_{\sigma}=\frac{1}{2}\left(\mathbf{0}^{\prime}, \boldsymbol{\omega}_{\sigma}^{\prime}\right)^{\prime}$, where $\boldsymbol{\omega}_{\sigma}$ is a vector of dimension $p+q+1$. We also have

$$
\boldsymbol{J}_{m}=\left(\begin{array}{cc}
E\left(\frac{1}{\sigma_{t}^{2}} \frac{\partial m_{t}\left(\boldsymbol{\varphi}_{0}\right)}{\partial \boldsymbol{\varphi}} \frac{\partial m_{t}\left(\boldsymbol{\varphi}_{0}\right)}{\partial \boldsymbol{\varphi}^{\prime}}\right) & \mathbf{0} \\
\mathbf{0} & \mathbf{0}
\end{array}\right), \quad \boldsymbol{J}_{\sigma}=\left(\begin{array}{cc}
E\left(\frac{1}{\sigma_{t}^{2}} \frac{\partial \sigma_{t}\left(\boldsymbol{\theta}_{0}\right)}{\partial \boldsymbol{\varphi}} \frac{\partial \sigma_{t}\left(\boldsymbol{\theta}_{0}\right)}{\partial \boldsymbol{\varphi}^{\prime}}\right) & \mathbf{0} \\
\mathbf{0} & \frac{1}{4} \boldsymbol{K}
\end{array}\right)
$$

where $\boldsymbol{K}=E\left(\frac{1}{\sigma_{t}^{4}} \frac{\partial \sigma_{t}^{2}\left(\boldsymbol{\theta}_{0}\right)}{\partial \boldsymbol{\vartheta}} \frac{\partial \sigma_{t}^{2}\left(\boldsymbol{\theta}_{0}\right)}{\partial \boldsymbol{\vartheta}^{\prime}}\right)$. The block-diagonality of $\boldsymbol{J}_{\sigma}$ follows from the fact that $\frac{1}{\sigma_{t}^{2}} \frac{\partial \sigma_{t}^{2}\left(\boldsymbol{\theta}_{0}\right)}{\partial \boldsymbol{\vartheta}}$ is an even function of the $\eta_{t-i}, i>0$, while $\frac{1}{\sigma_{t}^{2}} \frac{\partial \sigma_{t}^{2}\left(\boldsymbol{\theta}_{0}\right)}{\partial \boldsymbol{\varphi}}$ is an odd function. Hence, for some matrices $\boldsymbol{M}$ and $\boldsymbol{N}$ which we do not need to make explicit,

$$
\boldsymbol{J}=\left(\begin{array}{cc}
\boldsymbol{M} & \mathbf{0} \\
\mathbf{0} & \boldsymbol{K}
\end{array}\right), \quad \boldsymbol{\Sigma}=\left(\begin{array}{cc}
\boldsymbol{N} & \mathbf{0} \\
\mathbf{0} & \left(\kappa_{4}-1\right) \boldsymbol{K}^{-1}
\end{array}\right)
$$

Since Assumption A12* is satisfied, (38) holds and can be written $\boldsymbol{\omega}_{\sigma}^{\prime} \boldsymbol{K}^{-1} \boldsymbol{\omega}_{\sigma}=1$. We also have $\boldsymbol{H}(x)=\frac{x f(x)}{2}\left(\mathbf{0}^{\prime}, \boldsymbol{\omega}_{\sigma}^{\prime}\right)^{\prime}$, hence

$$
\boldsymbol{H}(x)^{\prime} \boldsymbol{\Sigma} \boldsymbol{H}(x)=\{x f(x)\}^{2} \frac{\kappa_{4}-1}{4}, \quad \boldsymbol{H}(x)^{\prime} \boldsymbol{\Lambda} \varrho(x)=\frac{x f(x)}{2} E\left\{\mathbb{1}_{\eta_{0}<x}\left(\eta_{0}^{2}-1\right)\right\} .
$$

The conclusion follows.

\section{E. Proof of the stochastic equicontinuity property in Lemma 9}

Noting that $U_{t}:=F\left(\eta_{t}\right)$ is uniformly distributed on $[0,1]$, we have

$$
e_{n}(x)=\frac{1}{\sqrt{n}} \sum_{t=1}^{n} 1_{U_{t} \leq F(x)}-F(x)=Y_{n}(F(x)), \quad Y_{n}(u)=\frac{1}{\sqrt{n}} \sum_{t=1}^{n} 1_{U_{t} \leq u}-u .
$$

Billingsley (1968) studied the modulus of continuity of $\left\{Y_{n}(u), u \in[0,1]\right\}$ and showed in $(22.13)$ that, for each $\varepsilon>0$ and $\eta>0$, there exists $\tau \in(0,1]$ such that

$$
P\left(\sup _{|u-v|<\tau}\left|Y_{n}(u)-Y_{n}(v)\right| \geq \varepsilon\right) \leq \eta
$$

for large $n$. For any $\varepsilon>0$ and $\delta>0$, we have

$$
P\left(\left|e_{n}\left(x_{n}\right)-e_{n}(x)\right| \geq \varepsilon\right) \leq P\left(\sup _{|y-x| \leq \delta}\left|e_{n}(y)-e_{n}(x)\right| \geq \varepsilon\right)+P\left(\left|x_{n}-x\right| \geq \delta\right) .
$$




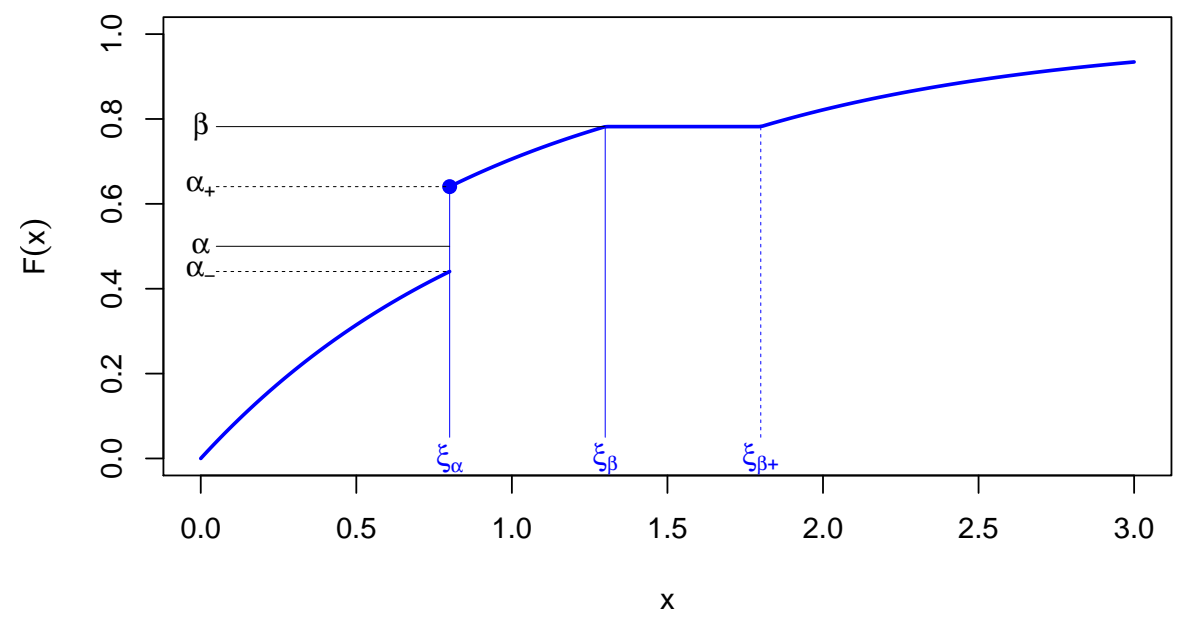

Fig. 3. Example of cdf.

The last probability tends to zero because $x_{n} \rightarrow x$ in probability. Now, note that

$$
P\left(\sup _{|y-x| \leq \delta}\left|e_{n}(y)-e_{n}(x)\right| \geq \varepsilon\right)=P\left(\sup _{|u-v| \leq F(\delta)}\left|Y_{n}(u)-Y_{n}(v)\right| \geq \varepsilon\right) \leq \eta
$$

when $n$ is large enough to satisfy (39) with $\tau>F(\delta)$. Since $\eta$ can be taken arbitrarily small, the result follows.

\section{F. Complements for Section 4}

\section{F.1. Proof and complements to Corollary 3}

The different items introduced in Corollary 3 and Lemma 17 are illustrated in Figure 3.

Proof of Corollary 3. The first convergence being a particular case of the second part of the theorem - since under $\mathbf{A 1 3}, \xi_{\alpha}=\xi_{\alpha}^{+}$- it will be sufficient to prove the latter. Let $\epsilon>0$. By definition of $\xi_{\alpha}$ and $\xi_{\alpha}^{+}$we have,

$$
F\left(\xi_{\alpha}-\epsilon\right)<\alpha-\delta \quad \text { and } \quad F\left(\xi_{\alpha}^{+}+\epsilon\right)>\alpha+\delta
$$

for some $\delta>0$. By Theorem 1 , assume $n$ large enough so that $\sup _{x \in \mathbb{R}}\left|\widehat{F}_{n}(x)-F(x)\right|<\delta$ a.s. It follows from (40) that

$$
\xi_{\alpha}-\epsilon \leq \widehat{\xi}_{n, \alpha} \leq \xi_{\alpha}^{+}+\epsilon
$$

Indeed, if $\xi_{\alpha}-\epsilon>\widehat{\xi}_{n, \alpha}$ then $F\left(\xi_{\alpha}-\epsilon\right) \geq F\left(\widehat{\xi}_{n, \alpha}\right)=F\left(\widehat{\xi}_{n, \alpha}\right)-\widehat{F}_{n}\left(\widehat{\xi}_{n, \alpha}\right)+\widehat{F}_{n}\left(\widehat{\xi}_{n, \alpha}\right) \geq \alpha-\delta$ which contradicts the first inequality in (40). Moreover, if $\xi_{\alpha}^{+}+\epsilon<\widehat{\xi}_{n, \alpha}$ then $F\left(\xi_{\alpha}^{+}+\epsilon\right) \leq \widehat{F}_{n}\left(\xi_{\alpha}^{+}+\epsilon\right)+\delta \leq$ 
$\alpha+\delta$ which contradicts the second inequality in (40). The strong convergence of $\widehat{\xi}_{n, \alpha}$ to the set $\left[\xi_{\alpha}, \xi_{\alpha}^{+}\right]$follows from (41). The conclusion follows.

The asymptotic behaviour of the empirical quantile when the residuals are replaced by innovations is given for the sake of comparison in the following lemma. Having been unable to find a reference for this result, we provide a proof for completeness. Let $\xi_{n, \alpha}$ the empirical $\alpha$-quantile of the innovations $\eta_{1}, \ldots, \eta_{n}$.

Lemma 17. For the iid sequence $\left(\eta_{t}\right)$ and $\alpha \in(0,1)$ we have:

(a) If $\xi_{\alpha}^{+} \neq \xi_{\alpha}$, then $\liminf \xi_{n, \alpha}=\xi_{\alpha}, \quad \lim \sup \xi_{n, \alpha}=\xi_{\alpha}^{+}, \quad$ a.s.

Moreover, $P\left(\xi_{n, \alpha} \leq \xi_{\alpha}\right)=1-P\left(\xi_{n, \alpha} \geq \xi_{\alpha}^{+}\right) \rightarrow 1 / 2$ as $n \rightarrow \infty$.

(b) If $\xi_{\alpha}^{+}=\xi_{\alpha}$ (Assumption A13), then $\xi_{n, \alpha} \rightarrow \xi_{\alpha}$ a.s. Moreover, letting $\alpha_{-}=P\left(\eta_{t}<\xi_{\alpha}\right)$ and $\alpha_{+}=P\left(\eta_{t} \leq \xi_{\alpha}\right)$ :

(i) If $\alpha>\alpha_{-}: P\left(\xi_{n, \alpha}<\xi_{\alpha}\right) \rightarrow 0$ as $n \rightarrow \infty$.

(ii) If $\alpha<\alpha_{+}: P\left(\xi_{n, \alpha} \leq \xi_{\alpha}\right) \rightarrow 1$ as $n \rightarrow \infty$.

(iii) If $\alpha=\alpha_{-}: P\left(\xi_{n, \alpha}<\xi_{\alpha}\right) \rightarrow 1 / 2$ as $n \rightarrow \infty$.

(iv) If $\alpha=\alpha_{+}: P\left(\xi_{n, \alpha} \leq \xi_{\alpha}\right) \rightarrow 1 / 2$ as $n \rightarrow \infty$.

Proof. The argument used in the proof of Corollary 3 shows that $\xi_{\alpha}-\epsilon \leq \xi_{n, \alpha} \leq \xi_{\alpha}^{+}+\epsilon$, noting that the usual Glivenko-Cantelli lemma does not require assumption A5. Because there are no $\eta_{t}$ 's in the interval $\left(\xi_{\alpha}, \xi_{\alpha}^{+}\right)$, it follows that, for sufficiently large $n$,

$$
\xi_{\alpha}-\epsilon \leq \xi_{n, \alpha} \leq \xi_{\alpha} \quad \text { or } \quad \xi_{\alpha}^{+} \leq \xi_{n, \alpha} \leq \xi_{\alpha}^{+}+\epsilon .
$$

If $\xi_{\alpha}^{+} \neq \xi_{\alpha}$, the last inequalities entail that $\left\{\liminf \xi_{n, \alpha}, \limsup \xi_{n, \alpha}\right\} \subset\left\{\xi_{\alpha}, \xi_{\alpha}^{+}\right\}, \quad$ a.s. Note that $\xi_{n, \alpha} \geq z \quad$ iff $\frac{1}{n} \sum_{t=1}^{n} \mathbb{1}_{\eta_{t}<z}<\alpha$. Moreover $P\left(\eta_{t}<\xi_{\alpha}^{+}\right)=P\left(\eta_{t} \leq \xi_{\alpha}\right)=F\left(\xi_{\alpha}\right) \geq \alpha$. Because $\xi_{\alpha}^{+}>\xi_{\alpha}$, we actually have $F\left(\xi_{\alpha}\right)=\alpha$. Therefore

$$
P\left(\xi_{n, \alpha} \geq \xi_{\alpha}^{+}\right)=P\left(\frac{1}{n} \sum_{t=1}^{n} \mathbb{1}_{\eta_{t}<\xi_{\alpha}^{+}}<\alpha\right)=P\left(Z_{n}<0\right),
$$

where

$$
Z_{n}=\frac{1}{\sqrt{n}} \sum_{t=1}^{n} \frac{\mathbb{1}_{\eta_{t}<\xi_{\alpha}^{+}}-\alpha}{\sqrt{\alpha(1-\alpha)}} \stackrel{\mathcal{L}}{\rightarrow} \mathcal{N}(0,1)
$$

The conclusion follows in case 1 .

Now, if $\xi_{\alpha}^{+}=\xi_{\alpha}$, the strong consistency of $\xi_{n, \alpha}$ follows from (42). To show the results in (a)-(d), the previous arguments can be adapted: for instance we have

$$
P\left(\xi_{n, \alpha} \geq \xi_{\alpha}\right)=P\left(\frac{1}{\sqrt{n}} \sum_{t=1}^{n} \frac{\mathbb{1}_{\eta_{t}<\xi_{\alpha}}-\alpha_{-}}{\sqrt{\alpha_{-}\left(1-\alpha_{-}\right)}}<\sqrt{n} \frac{\alpha-\alpha_{-}}{\sqrt{\alpha_{-}\left(1-\alpha_{-}\right)}}\right)
$$



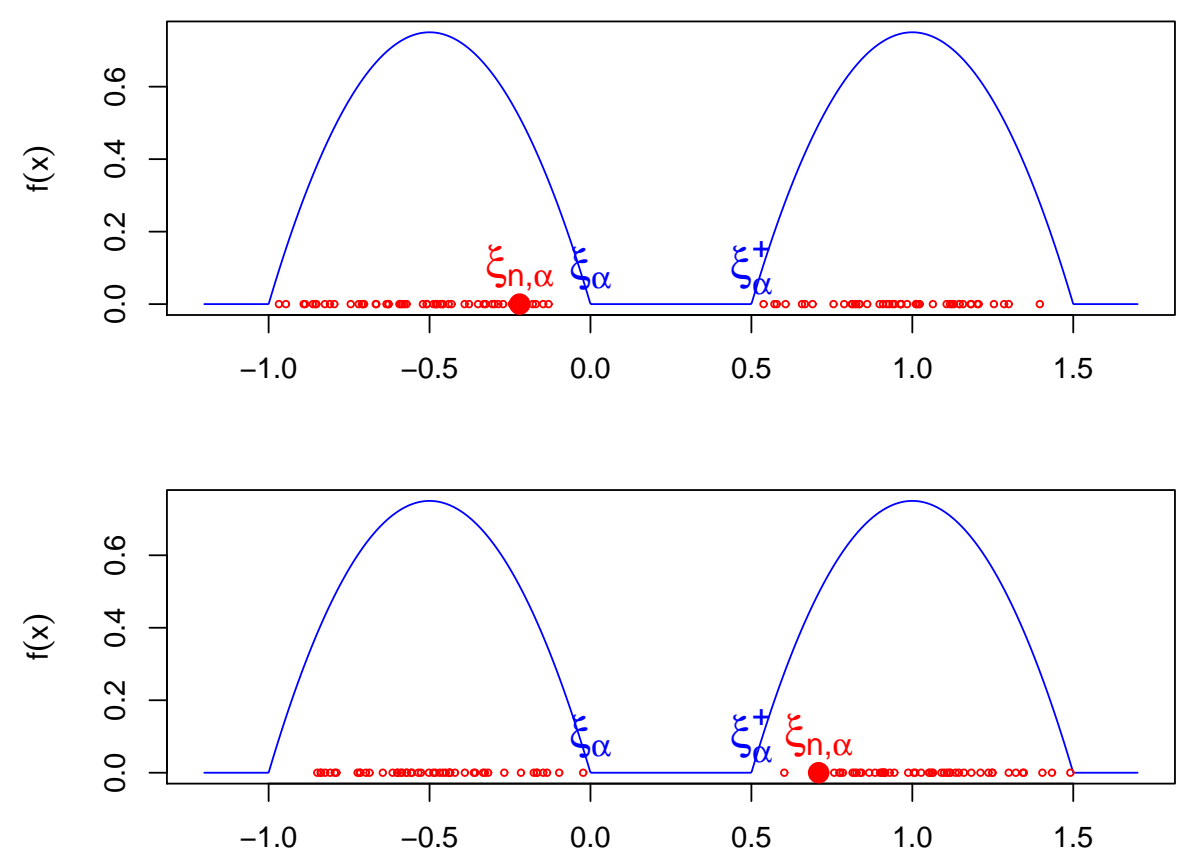

Fig. 4. For $\alpha=1 / 2$, computations of $\xi_{n, \alpha}$ for two simulations of length 101 of a density which does not satisfy A13.

from which (a) and (c) can be deduced. The arguments for (b) and (d) are similar, using the characterization: $\xi_{n, \alpha} \leq z$ iff $\frac{1}{n} \sum_{t=1}^{n} \mathbb{1}_{\eta_{t} \leq z} \geq \alpha$.

The first part of this lemma shows that, for iid observations, (7) is replaced by

$$
\left[\lim \inf \xi_{n, \alpha}, \lim \sup \xi_{n, \alpha}\right]=\left[\xi_{\alpha}, \xi_{\alpha}^{+}\right] \quad \text { a.s. }
$$

In particular, the empirical quantile does not converge when Assumption A13 is not satisfied, as illustrated in Figure 4. The second part shows, in particular, that if $\alpha_{-}<\alpha<\alpha_{+}$then $P\left(\xi_{n, \alpha}=\xi_{\alpha}\right)$ tends to 1 . On the other hand, when $\alpha_{-}=\alpha_{+}$(no mass at $\left.\xi_{\alpha}\right)$ then $P\left(\xi_{n, \alpha}=\xi_{\alpha}\right)$ tends to 0 .

When $\mathbf{A 1 3}$ does not hold, not only $\xi_{n, \alpha}$ but also the sample quantile $\widehat{\xi}_{n, \alpha}$ of the residuals may not converge to $\xi_{\alpha}$. For instance, in the constant scale model, $\epsilon_{t}=\sigma \eta_{t}$, we have $\widehat{\eta}_{t}=\frac{\sigma}{\widehat{\sigma}} \eta_{t}$ where the ratio converges to 1 and thus both $\xi_{n, \alpha}$ and $\widehat{\xi}_{n, \alpha}=\frac{\sigma}{\sigma} \xi_{n, \alpha}$ do not converge. 


\section{References}

Andrews, D. W. (1994) Asymptotics for semiparametric econometric models via stochastic equicontinuity. Econometrica $62,43-72$.

Bai, J. (1994) Weak convergence of the sequential empirical processes of residuals in ARMA Models. The Annals of Statistics 22, 2051-2061.

Berkes, I. and L. Horváth (2002). Empirical processes of residuals. In Empirical process techniques for dependent data (pp. 195-209). Birkhäuser, Boston, MA.

Berkes, I. and L. Horváth (2003) Limit results for the empirical process of squared residuals in GARCH models. Stochastic Processes and their Applications 105, 271-298.

Billingsley, P. (1961) The Lindeberg-Lévy theorem for martingales. Proceedings of the American Mathematical Society 12, 788-792.

Billingsley, P. (1968) Convergence of probability measures. Wiley, New York.

Boldin, M. V. (1982) Estimation of the distribution of noise in an autoregressive scheme. Theory Probabability and Applications 27 866-871.

Boldin, M. V. (1998) On residual empirical distribution functions in ARCH models with applications to testing and estimation. Mitt. Math. Sem. Giessen 235, 49-66.

Burkholder, D.L. (1973) Distribution function inequalities for martingales. The Annals of Probability 1, 19-42.

Feigin, P.D. and R.L. Tweedie (1985) Random coefficient autoregressive processes: a Markov chain analysis of stationarity and finiteness of moments. Journal of Time Series Analysis 6, $1-14$.

Francq, C. and J-M. Zakoïan (2004) Maximum likelihood estimation of pure GARCH and ARMA-GARCH processes. Bernoulli 10, 605-637.

Francq, C. and J-M. Zakoïan (2013a) Optimal predictions of powers of conditionally heteroscedastic processes. Journal of the Royal Statistical Society: Series B 75, 345-367.

Francq, C. and J-M. Zakoïan (2013b) Inference in nonstationary asymmetric GARCH models. The Annals of Statistics 41, 1970-1998.

Francq, C. and J-M. Zakoïan (2015) Risk parameter estimation in volatility models. Journal of Econometrics 184, 158-173. 
Francq, C. and J-M. Zakoïan (2019) GARCH Models: Structure, Statistical Inference and Financial Applications. 2nd Edition. John Wiley.

Hall, P., and C.C. Heyde (1980) Martingale limit theory and its application. Academic press, New York.

Hallin, M., Paindaveine, D. and M. Šiman (2010) Multivariate quantiles and multipleoutput regression quantiles: From $L_{1}$ optimization to halfspace depth. The Annals of Statistics $38,635-669$.

Hoga, Y. (2019) Confidence intervals for conditional tail risk measures in ARMA-GARCH models. Journal of Business and Economic Statistics 37, 613-624.

Horváth, L., Kokoszka, P. and G. Teyssière (2001) Empirical process of the squared residuals of an ARCH sequence. The Annals of Statistics 29, 445-469.

Koul, H. L., and S. Ling (2006) Fitting an error distribution in some heteroscedastic time series models. The Annals of Statistics 34, 994-1012.

Kreiss, J. P. (1987). On adaptive estimation in stationary ARMA processes. The Annals of Statistics 15, 112-133.

Kreiss, J. P. (1991). Estimation of the distribution function of noise in stationary processes. Metrika 38, 28-297.

Kulperger, R., and H. Yu (2005) High moment partial sum processes of residuals in GARCH models and their applications. The Annals of Statistics 33, 2395-2422.

Lee, S. and M. Taniguchi (2005) Asymptotic theory for ARCH-SM models: LAN and residual empirical processes. Statistica Sinica 15, 215-234.

Ling, S. (1998) Weak convergence of the sequential empirical processes of residuals in nonstationary autoregressive models. The Annals of Statistics 26, 741-754.

Ling, S. (2007) A double AR(p) model: structure and estimation. Statistica Sinica 17, 161-175.

Robinson, P.M. Asymptotically efficient estimation in the presence of heteroskedaticity of unknown form. Econometrica 55, 875-891.

Shorack, G.R. and J.A. Wellner (1986) Empirical processes with applications to statistics. Wiley, New-York. 
Stute, W. (2001) Residual analysis for $\operatorname{ARCH}(p)$-time series. Test 10, 393-403.

Stute, W. and G. Schumann (1980) A general Glivenko-Cantelli theorem for stationary sequences of random observations. Scandinavian Journal of Statistics 7, 102-104.

Wang, G., Zhu, K., Li, G. and W. K. Li (2019) Hybrid quantile estimation for asymmetric power GARCH models. arXiv:1911.09343.

Zheng, Y., Zhu, Q., Li, G., and Z. Xiao (2018) Hybrid quantile regression estimation for time series models with conditional heteroscedasticity. Journal of the Royal Statistical Society: Series B 80, 975-993.

Zhu, Q. and G. Li (2019) Quantile double autoregression. Available at SSRN 3335753. 